\title{
Article
}

\section{Synthesis of New Highly Functionalized 1H-Indole-2-carbonitriles via Cross-Coupling Reactions}

\author{
Asma Hrizi ${ }^{1,2,3}$, Manon Cailler ${ }^{1}$, Moufida Romdhani-Younes ${ }^{2,3}$, Yvan Carcenac ${ }^{1}$ and Jérôme Thibonnet ${ }^{1, *(1)}$ \\ 1 Laboratoire Synthèse et Isolement de Molécules BioActives EA 7502 SIMBA, Department of Chemistry, \\ Faculté des Sciences et Techniques de Tours, Université de Tours, Parc de Grandmont, 37200 Tours, France; \\ asma.hrizi1993@gmail.com (A.H.); manon.cailler@gmail.com (M.C.); yvan.carcenac@univ-tours.fr (Y.C.) \\ 2 Department of Chemistry, Faculté des Sciences de Bizerte, Université de Carthage, Zarzouna, Bizerte 7000, \\ Tunisia; moufida.romdhani@gmail.com \\ 3 Laboratoire de Chimie (Bio) Organique, Département de Chimie, Faculté des Sciences de Tunis, Campus \\ Universitaire, Université de Tunis El Manar, Structurale et de Polymères-Synthèse et Études \\ Physico-Chimiques (LR99ES14), Tunis 2092, Tunisia \\ * Correspondence: jerome.thibonnet@univ-tours.fr; Tel.: +33-0-247-367-041
}

Citation: Hrizi, A.; Cailler, M.; Romdhani-Younes, M.; Carcenac, Y.; Thibonnet, J. Synthesis of New Highly Functionalized $1 H$-Indole-2-carbonitriles via Cross-Coupling Reactions. Molecules 2021, 26, 5287. https://doi.org/ $10.3390 /$ molecules 26175287

Academic Editor: Artur M. S. Silva

Received: 15 July 2021

Accepted: 27 August 2021

Published: 31 August 2021

Publisher's Note: MDPI stays neutral with regard to jurisdictional claims in published maps and institutional affiliations.

Copyright: (c) 2021 by the authors. Licensee MDPI, Basel, Switzerland. This article is an open access article distributed under the terms and conditions of the Creative Commons Attribution (CC BY) license (https:/ / creativecommons.org/licenses/by/ $4.0 /)$.

\begin{abstract}
An approach for the preparation of polysubstituted indole-2-carbonitriles through a crosscoupling reaction of compounds 1-(but-2-ynyl)-1H-indole-2-carbonitriles and 1-benzyl-3-iodo- $1 \mathrm{H}$ indole-2-carbonitriles is described. The reactivity of indole derivatives with iodine at position 3 was studied using cross-coupling reactions. The Sonogashira, Suzuki-Miyaura, Stille and Heck cross-couplings afforded a variety of di-, tri- and tetra-substituted indole-2-carbonitriles.
\end{abstract}

Keywords: $1 H$-indole-2-carbonitriles; Sonogashira; Suzuki-Miyaura; Heck; Stille

\section{Introduction}

Indole skeletons exist as key building blocks in drugs, natural products, pharmaceuticals, alkaloids and agrochemicals and exhibit potent and wide-ranging biological activities [1-5]. The indole scaffold represents probably one of the most important structural subunits for the discovery of new drug candidates [6-9]. In particular, the derivatives of 2-cyanoindoles gained considerable attention in recent years because of their great importance in biological sciences, and they are also of interest thanks to this nitrile function [10-12]. The 2-cyanoindole unit is an example of structural motif building blocks and effective precursors for the synthesis of various indole-fused polycycles [13-19], substituted 2-cyanoindoles [20-24], addition to nitriles [25,26] and indole heterocycle substitution $[27,28]$. These compounds exhibit a wide range of biological activities (Figure 1). They are widely used in medicinal chemistry and pharmacological research as antagonist molecules. For example, adrenergic antagonist A [14] is a drug that inhibits the function of adrenergic receptors. There are also $\alpha$-adrenoreceptors that are located on vascular smooth muscle. Antagonists reduce or block the signals of agonists. They can be drugs, which are added to the body for therapeutic reasons, or endogenous ligands. Analog $\mathbf{D}$ [27] of firefly luciferin is a compound of the class of luciferins, light-emitting chemical compounds. It is found in many species of fireflies (Lampyridae). It is the substrate of luciferase, an enzyme that catalyzes its oxidation into oxyluciferin with concomitant hydrolysis of a molecule of ATP into AMP and PPi accompanied by the emission of a photon of yellow light characteristic of these insects. NMDA receptor antagonists E [25] are a class of drugs that work to antagonize or inhibit the action of the $N$-Methyl-D-aspartate receptor (NMDAR). They are commonly used as anesthetics for animals and humans; the state of anesthesia they induce is referred to as dissociative anesthesia. The dopamine $\mathrm{D}_{4}$ receptor $\left(\mathrm{D}_{4} \mathrm{R}\right) \mathrm{F}$ [20] plays important roles in cognition, attention and decision making. Novel $\mathrm{D}_{4} \mathrm{R}$-selective ligands have promise in medication development for neuropsychiatric conditions, including Alzheimer's disease and substance use disorders. Prostaglandin E2 (PGE2) 
modulator G [21], subtype (EP2), which is a metabolite of arachidonic acid that binds with and regulates cellular responses to PGE2, is associated with numerous physiological and pathological events in a wide range of tissues. As a stimulatory $G$ protein-coupled receptor, PGE2-induced EP2 activation can activate adenylate cyclase, leading to increased cytoplasmic cAMP levels and activation of protein kinase A. Finally, compound $\mathbf{H}$ [22] is considered as an antiarrhythmic agent. Additionally, the cyano group is a valuable and readily available functional group for the preparation of various functional groups such as amines, amides, esters, ketones and their carboxyl derivatives [29].

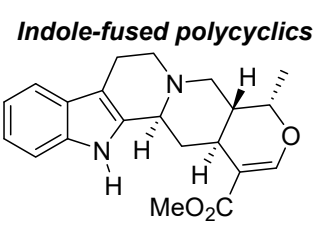

A Adrenergic receptor antagonist

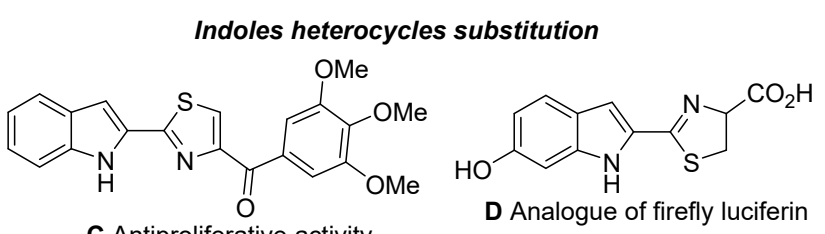

C Antiproliferative activity<smiles>[R]c1c2ccccc2n2ccc3c(c12)NNc1ccccc1-3</smiles>

B Homofascaplysin B and C $\mathrm{R}=\mathrm{CHO}$ or $\mathrm{C}(\mathrm{O}) \mathrm{CO}_{2} \mathrm{Me}$

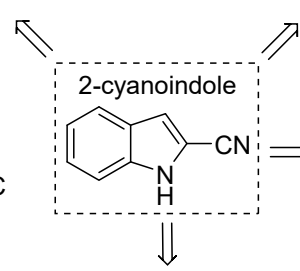

Substituted 2-cyanoindoles

Addition to nitriles

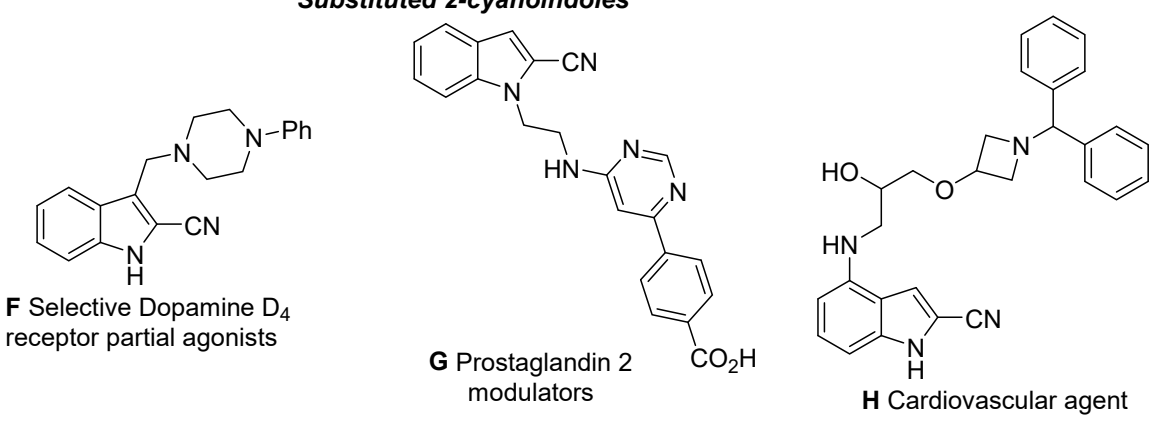

F Selective Dopamine $D_{4}$ receptor partial agonists<smiles>N#Cc1cc2c(NCC(O)COC3CN(C(c4ccccc4)c4ccccc4)C3)cccc2[nH]c1=O</smiles>

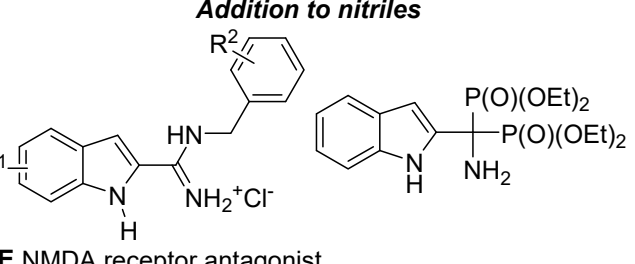

E NMDA receptor antagonist

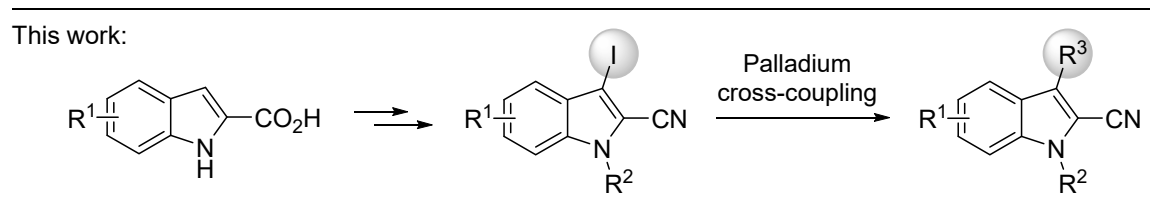

Figure 1. Biologically relevant compounds, featuring 2-cyanoindole cores.

Due to their importance, the development of efficient methodologies for the preparation and functionalization of various cyanoindoles has been the subject of intense research efforts. Direct incorporation of the nitrile function to substituted indoles has been accomplished through a variety of methods. These methods involved various sources of a cyano group including: acetonitrile [30], tert-butylisocyanide [31], nitromethane [32], benzyl cyanide [33], Beller's NCTS ( $N$-cyano- $N$-phenyl- $p$-toluenesulfonamide) [34] or $\mathrm{Zn}(\mathrm{CN})_{2}[35,36]$.

Palladium-catalyzed cross-coupling reactions are among the most successful transformations in organic synthesis. Thanks to all research work carried out over the years, a large variety of $\mathrm{C}-\mathrm{C}$ and $\mathrm{C}-\mathrm{X}$ bond formations and numerous highly active catalytic combinations are currently available [37-41]. The broad interest of this cross-coupling methodology is thus found in many fields of application [42,43]. Driven by our interest in the preparation of substituted 2-cyanoindoles and in conjunction with our successful previous research on palladium cross-coupling reactions, we explored the reactivity of 3-iodo-indole-2-carbonitrile of the residual iodine. This approach allowed the preparation of novel substituted 2-cyanoindoles in position 3 (Figure 1). 
The aim of this work was to synthesize new $1 H$-indole-2-carbonitrile derivatives, which could also be useful for drug design. Moreover, the substitution in this type of product in position 3 is important for the development of new molecules with biological interests. The reactivity of iodine in this position was studied using some cross-coupling reactions such as Sonogashira, Suzuki-Miyaura, Stille and Heck reactions, which provided a wide variety of molecules. We report the preparation of 3-iodo-2-carbonitrile derivatives as precursors through the use of the $\mathrm{C}-\mathrm{I}$ bond in coupling reactions to access a molecular diversity (Figure 1).

\section{Results and Discussion}

\subsection{Synthesis of 1 H-Indole-2-carbonitriles}

We began our studies with the reaction of thionyl chloride of $\mathbf{1 a}-\mathbf{d}$ with a catalytic amount of DMF, which gives access to acyl chloride that reacts with a solution of $25 \%$ ammonia to give $1 H$-indole-2-carboxamide derivatives $\mathbf{2 a - d}$ in good yields (Scheme 1 ). The reaction with phosphorus $(\mathrm{V})$ oxychloride on the carboxamide afforded the corresponding $1 \mathrm{H}$-indole-2-carbonitriles derivatives $\mathbf{3 a}-\mathbf{d}$ in good yields (65-85\%), following a modified procedure (see Experimental Section) [28].

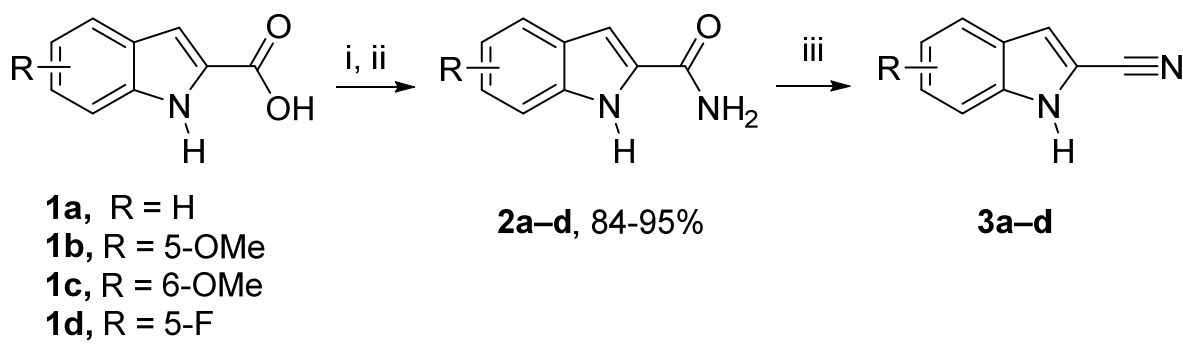

\section{Selected examples}<smiles>N#Cc1cc2ccccc2[nH]1</smiles><smiles>COc1ccc2c(c1)cc(C#N)n2[18OH]</smiles>

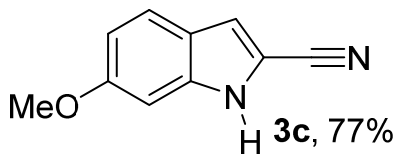<smiles>N#Cc1cc2cc(F)ccc2[nH]1</smiles>

Scheme 1. Reagents and conditions: (i) $\mathrm{SOCl}_{2}$ (1.2 equiv.), DMF cat., dry $\mathrm{CHCl}_{3}$, reflux, $24 \mathrm{~h}$; (ii) $\mathrm{NH}_{3} 25 \%$, room temperature (rt), 2 h; (iii) $\mathrm{POCl}_{3}$ (4 equiv.), dry $\mathrm{CHCl}_{3}$, reflux, $3 \mathrm{~h}$.

\subsection{Synthesis of 1-(3-Phenylprop-2-yn-1-yl)-1H-indole-2-carbonitrile Derivatives}

Propargylation reaction ( $\mathrm{NaH}$ then propargyl bromide in DMF) provides the desired 1-(prop-2-yn-1-yl)-1H-indole-2-carbonitrile $4 \mathbf{a}$ in a yield of $75 \%[10,44]$. An efficient method for palladium-catalyzed homocoupling reaction of terminal alkyne in the synthesis of $1 \mathrm{H}$-indole-2-carbonitrile derivatives was carried out (Scheme 2). In the presence of $10 \mathrm{~mol} \%$ of $\mathrm{PdCl}_{2}\left(\mathrm{PPh}_{3}\right)_{2}, 10 \mathrm{~mol} \%$ of $\mathrm{CuI}$ and 3 equiv. of $\mathrm{Et}_{3} \mathrm{~N}$, homocoupling of various aromatic iodines gave the corresponding dissymmetric alkynes 1-(3-phenylprop-2-yn-1-yl)$1 \mathrm{H}$-indole-2-carbonitrile derivatives 5a-f in moderate to good yields (64-90\%) [16]. This Sonogashira cross-coupling reaction was run with various substitutions using electron donor groups and electron-withdrawing groups on the aromatic ring, either in ortho, meta or para. 


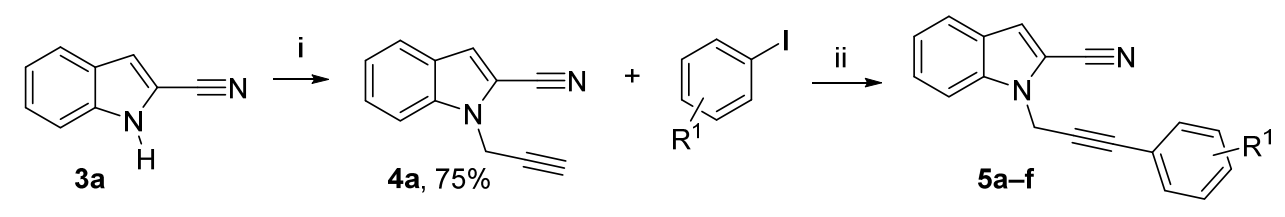

Selected examples
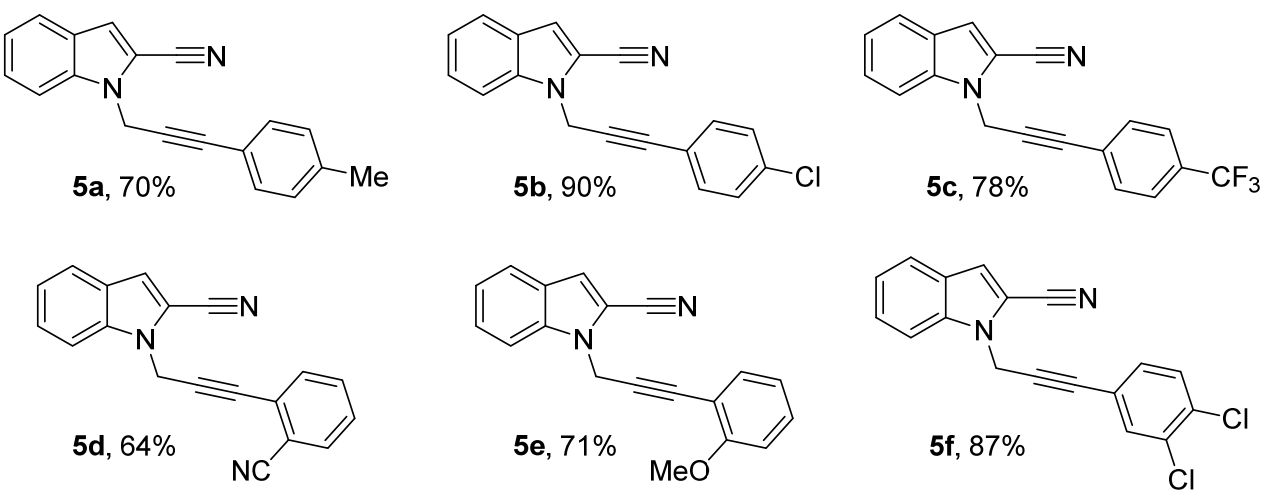

Scheme 2. Reagents and conditions: (i) $\mathrm{NaH}$ (1.3 equiv.), DMF, $0{ }^{\circ} \mathrm{C}, 15 \mathrm{~min}$, propargyl bromide 80 wt. \% in toluene (1.3 equiv.), $0{ }^{\circ} \mathrm{C}$ to rt, $4 \mathrm{~h}$; (ii) $4 \mathrm{a}$ (1 equiv.), aryl iodide (1.3 equiv.), $\mathrm{Et}{ }_{3} \mathrm{~N}$ ( 3 equiv.), $\mathrm{CuI}(10 \mathrm{~mol} \%), \mathrm{PdCl}_{2}\left(\mathrm{PPh}_{3}\right)_{2}(10 \mathrm{~mol} \%), \mathrm{DMF}, \mathrm{rt}$, overnight.

\subsection{Synthesis of 1-Benzyl-3-iodo-1H-indole-2-carbonitrile Derivatives}

A new series of 2-cyanoindole was also synthesized with an alkynyl substituent in position 3. First, aromatic electrophilic substitution in position 3 by iodine with potassium hydroxide was undertaken (Scheme 3) [45]. The resulting iodine derivatives $\mathbf{6 a}-\mathbf{d}$ were obtained in yields above $80 \%$. Finally, a protection reaction was carried out with benzyl bromide to give the corresponding 1-benzyl-3-iodo- $1 \mathrm{H}$-indole-2-carbonitrile derivatives 7a-d in good yields (71-92\%) [46].

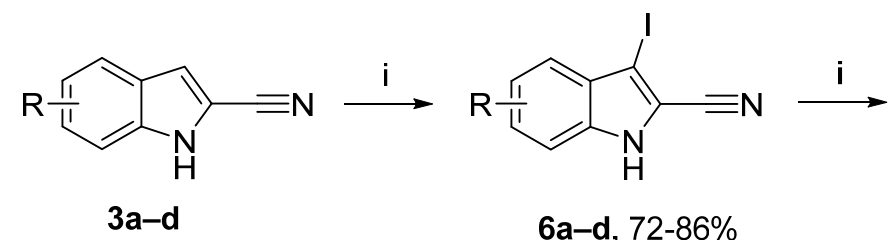<smiles>N#Cc1c(I)c2cc[R]cc2n1Cc1ccccc1</smiles>

$7 \mathrm{a}, \mathrm{R}=\mathrm{H}, 71 \%$ $7 \mathrm{~b}, \mathrm{R}=5-\mathrm{OMe}, 81 \%$ $7 \mathrm{c}, \mathrm{R}=6-\mathrm{OMe}, 92 \%$

$7 d, R=5-F, 76 \%$

Scheme 3. Reagents and conditions: (i) 3a-d (1 equiv.), $\mathrm{KOH}$ (3.6 equiv.), DMF, rt, $30 \mathrm{~min}$, then $\mathrm{I}_{2}$ (1 equiv.), $0{ }^{\circ} \mathrm{C}$ to rt, $4 \mathrm{~h}$; (ii) $\mathrm{NaH}$ (1.2 equiv.), DMF, $0{ }^{\circ} \mathrm{C}, 30 \mathrm{~min}$, benzyl bromide (1.3 equiv.), $0{ }^{\circ} \mathrm{C}$ to rt.

\subsection{Sonogashira Reaction on the 1-Benzyl-3-iodo-1H-indole-2-carbonitrile Derivatives}

Sonogashira cross-coupling was performed using $\mathbf{7 a - d}$ as substrates (Scheme 4) [47,48]. The alkynyl substituent in position 3 has been formed with phenylacetylene derivatives, $10 \mathrm{~mol} \%$ of palladium (II) and $10 \mathrm{~mol} \%$ of copper iodide. The reaction of 1-benzyl-3iodo- $1 \mathrm{H}$-indole-2-carbonitrile with a variety of aromatic alkynes containing both electrondonating and electron-withdrawing substituents was also examined. Thus, compounds $\mathbf{8} \mathbf{a}-\mathbf{j}$ were obtained in moderate to good yields (69-90\%), and this reaction was carried out with various substituents, either in ortho (F, OMe), meta $(\mathrm{Me})$ or para (Et, F, OMe) and also with pyridine derivatives. These products were purified either by crystallization or by chromatography on silica gel. 

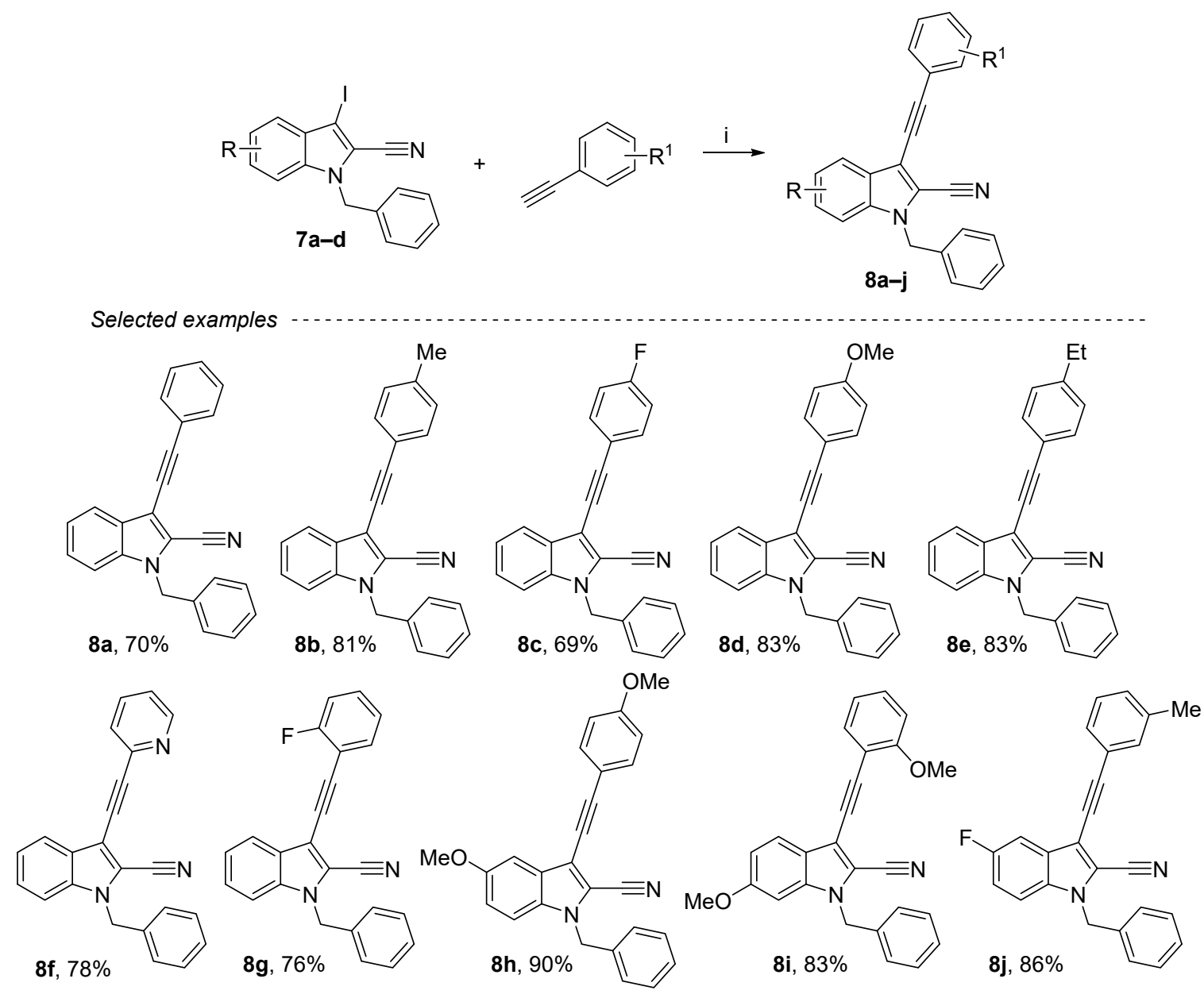

Scheme 4. Reagents and conditions: (i) 4a (1 equiv.), aryl iodide derivative (1.3 equiv.), $\mathrm{Et}_{3} \mathrm{~N}$ (3 equiv.), CuI (10 mol\%), $\mathrm{PdCl}_{2}\left(\mathrm{PPh}_{3}\right)_{2}(10 \mathrm{~mol} \%), \mathrm{DMF}, \mathrm{rt}$, overnight.

Previously, our group reported on the access to iodo-imidazodipyridines from imidazopyridine-2-carbonitriles promoted by Grignard reagent in the presence of iodine and $\mathrm{ZnI}_{2}$ through a 6-endo-dig cyclization [49]. We wished to extend these results to the indole core. Some additional tests of magnesium ethyl bromide on compound $\mathbf{8 b}$ either in diethyl ether or in cyclopentylmethylether (CPME) were made. Changing reaction times and temperatures were carried out. The 6-endo-dig cyclization product was not isolated, and the addition of Grignard reagent on the cyano group provided the corresponding ketone in a very low yield (4\%). However, a test with $n$-BuLi as the organometallic agent yielded the corresponding ketone $\mathbf{8} \mathbf{b}^{\mathbf{\prime}}$ with a full conversion rate, and no cyclization product was observed (Scheme 5). Further efforts to studies on the mechanism and synthetic applications for this type of cyclization are underway in our laboratory. 
Previous work: serie imidazopyridine-2-carbonitriles

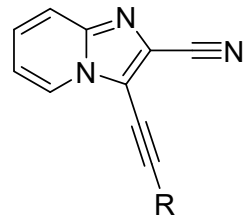

$\mathrm{R}=c-\mathrm{Pr}, \mathrm{Ph}, n-\mathrm{Bu}$

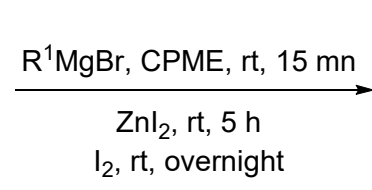

iodo-imidazodipyridines

4 examples, $26-71 \%$
$\mathrm{R}^{1}=\mathrm{Me}, \mathrm{Et}, \mathrm{Ph}$<smiles>[R]c1nc([R])c2nc3ccccn3c2c1I</smiles>

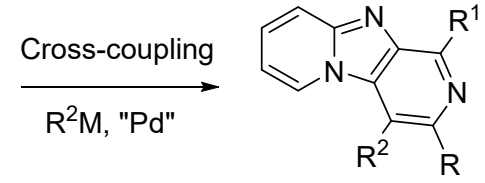

7 examples, $50-98 \%$

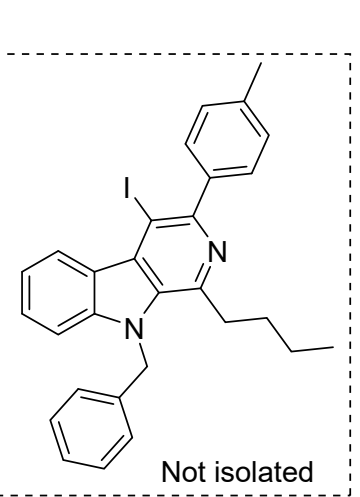

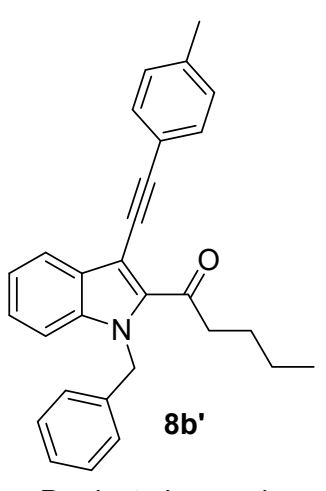

Product observed

Scheme 5. Addition of $n$-BuLi on compound $\mathbf{8 b}$ : access to ketone $\mathbf{8} \mathbf{b}^{\prime}$.

\subsection{Suzuki Reaction on the 1-Benzyl-3-iodo-1H-indole-2-carbonitrile Derivatives}

Suzuki cross-coupling is one of the most efficient methods for the construction of $\mathrm{C}-\mathrm{C}$ bonds. Although several other methods are available for this purpose, the Suzuki cross-coupling reaction, which produces biaryls, has proven to be the most popular in recent times. The key advantages of this coupling are the commercial availability of diverse boronic acids that are environmentally safer than other organometallic reagents. Another cross-coupling on nitrile compounds was also performed. The Suzuki reaction was carried out herein with a slight excess of boronic acid using $\mathrm{NaHCO}_{3}$ as base and tetrakistriphenylphosphine (10 $\mathrm{mol} \%)$ as a catalyst in toluene/water mixture as a solvent (Scheme 6) [50-52]. Several compounds were synthesized with different ortho-, meta- or para-aryls substituted by electron donor groups $(\mathrm{Me}, \mathrm{Et}, \mathrm{tBu}, \mathrm{OMe})$ or electron-drawing groups $(\mathrm{F}, \mathrm{Cl})$ and with naphthalene derivative. Good yields (79-93\%) were also obtained when substituted phenyl groups were used in this cross-coupling reaction $(\mathbf{9 a}-\mathbf{p})$. These products were purified either by crystallization or by chromatography on silica gel. 

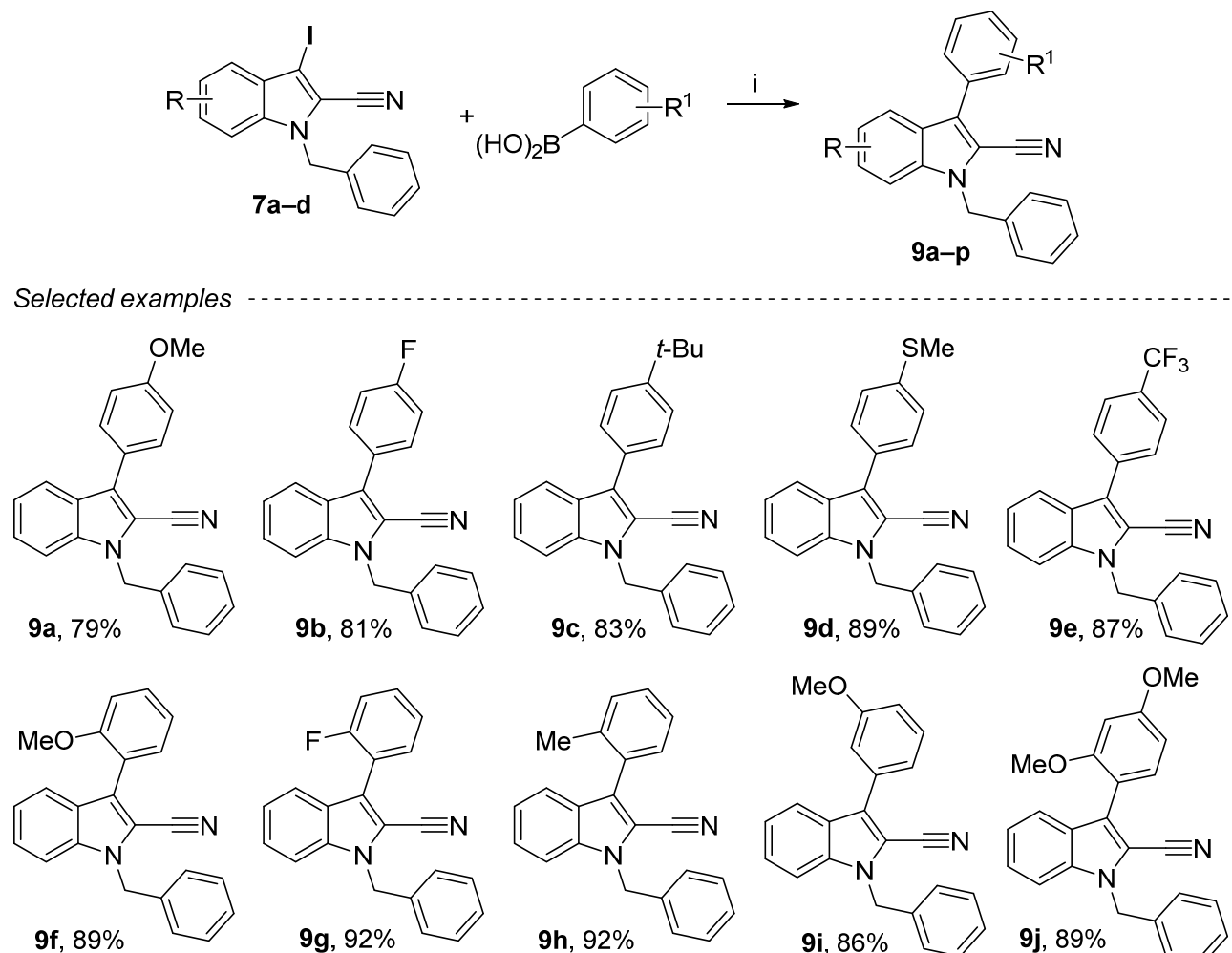<smiles>COc1cccc(-c2c(C#N)n(Cc3ccccc3)c3ccccc23)c1</smiles><smiles>COc1ccc(-c2c(C#N)n(Cc3ccccc3)c3ccccc23)c(OC)c1</smiles><smiles>N#Cc1c(-c2ccc3c(c2)OCCO3)c2ccccc2n1Cc1ccccc1</smiles><smiles>COc1ccc2ccc(-c3c(C#N)n(Cc4ccccc4)c4ccccc34)cc2c1</smiles><smiles>COc1ncccc1-c1c(C#N)n(Cc2ccccc2)c2ccccc12</smiles><smiles>COc1ccc(-c2c(C#N)n(Cc3ccccc3)c3ccc(OC)cc23)cc1</smiles><smiles>COc1cccc(-c2c(C#N)n(Cc3ccccc3)c3cc(OC)ccc23)c1</smiles><smiles>COc1cccc(-c2c(C#N)n(Cc3ccccc3)c3ccc(F)cc23)c1</smiles>

Scheme 6. Reagents and conditions: (i) $7 \mathrm{a}-\mathrm{d}$ (1 equiv.), boronic acids (1.2 equiv.), sat. aq. $\mathrm{NaHCO}_{3}, \mathrm{EtOH} /$ toluene $=3 / 2$, $\mathrm{Pd}\left(\mathrm{PPh}_{3}\right)_{4} 10 \mathrm{~mol} \%, 130{ }^{\circ} \mathrm{C}, 4 \mathrm{~h}$.

\subsection{Heck Reaction on the 1-Benzyl-3-iodo-1H-indole-2-carbonitrile Derivatives}

Among all palladium-catalyzed cross-couplings, the Mizoroki-Heck reaction allows a direct coupling between heteroatomic compounds and alkenes [53]. This reaction allowed the creation of a sigma bond between two $\mathrm{sp}^{2}$-hybridized carbon atoms through a C-H activation. Compared to other cross-coupling processes, this one presents practical and economic advantages such as using simple and readily available materials. Under conditions (DMF, $80{ }^{\circ} \mathrm{C}, 24 \mathrm{~h}$ ), coupling of 1-benzyl-3-iodo- $1 \mathrm{H}$-indole-2-carbonitrile derivatives in the presence of $\mathrm{KOAc}$ (6 equiv.), $n-\mathrm{Bu}_{4} \mathrm{NCl}$ (2 equiv.), $\mathrm{Pd}(\mathrm{OAc})_{2}(4 \mathrm{~mol} \%$ ) with diverse olefins afforded a series of cyanoindoles substituted in position 3 (10a-f) in excellent yields (81-92\%) (Scheme 7). The E-configuration products were mainly obtained except for the acrylonitrile, for which a mixture of two isomers $(E / Z=75 / 25)$ was observed. 


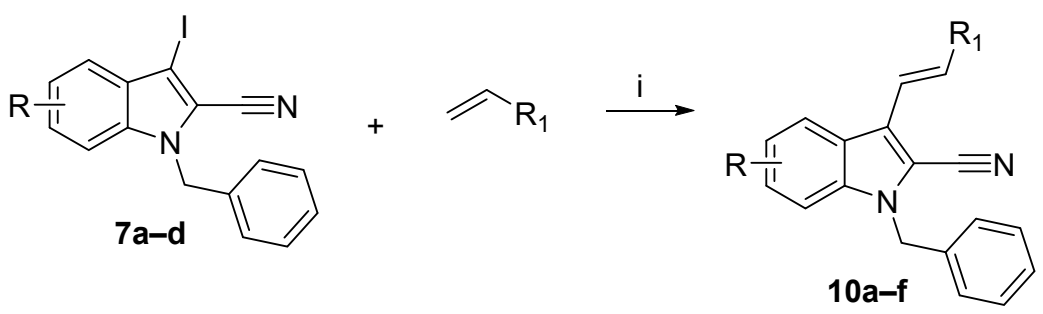

Selected examples<smiles>CC(=O)C=Cc1c(C#N)n(Cc2ccccc2)c2ccccc12</smiles><smiles>N#Cc1c(/C=C/c2ccccc2)c2ccccc2n1Cc1ccccc1</smiles><smiles></smiles><smiles>COc1ccc2c(c1)c(/C=C/C(C)=O)c(C#N)n2Cc1ccccc1</smiles><smiles>COc1ccc2c(C=CC(C)=O)c(C#N)n(Cc3ccccc3)c2c1</smiles><smiles>CC(=O)C=Cc1c(C#N)c2cc(F)ccc2n1Cc1ccccc1</smiles>

Scheme 7. Reagents and conditions: (i) $7 \mathbf{a}-\mathbf{d}$ ( 1 equiv.), alkenes (1.2 equiv.), KOAc (6 equiv.), $n$ $\mathrm{Bu}_{4} \mathrm{NCl}$ (2 equiv.), $\mathrm{Pd}(\mathrm{OAc})_{2} 4 \mathrm{~mol} \%$, DMF, $80{ }^{\circ} \mathrm{C}, 24 \mathrm{~h}$.

\subsection{Stille Reaction on the 1-Benzyl-3-iodo-1H-indole-2-carbonitrile Derivatives}

Stille coupling was performed using an organotin compound and a catalytic amount of dichlorobis(acetonitrile)palladium(II) in DMF at $40^{\circ} \mathrm{C}$ (Scheme 8) [54,55]. Under these conditions, the coupling with compound 7a was successfully achieved, and two examples of coupling products (11b and 11c) were obtained in low to moderate yields (35-40\%). No coupling product with tributyl(vinyl)stannane could be obtained (11a, $0 \%)$. Finally, these compounds were purified by column chromatography with a stationary phase composed of $10 \%$ powdered anhydrous $\mathrm{K}_{2} \mathrm{CO}_{3}$ and silica to remove all traces of organotin impurities [56].
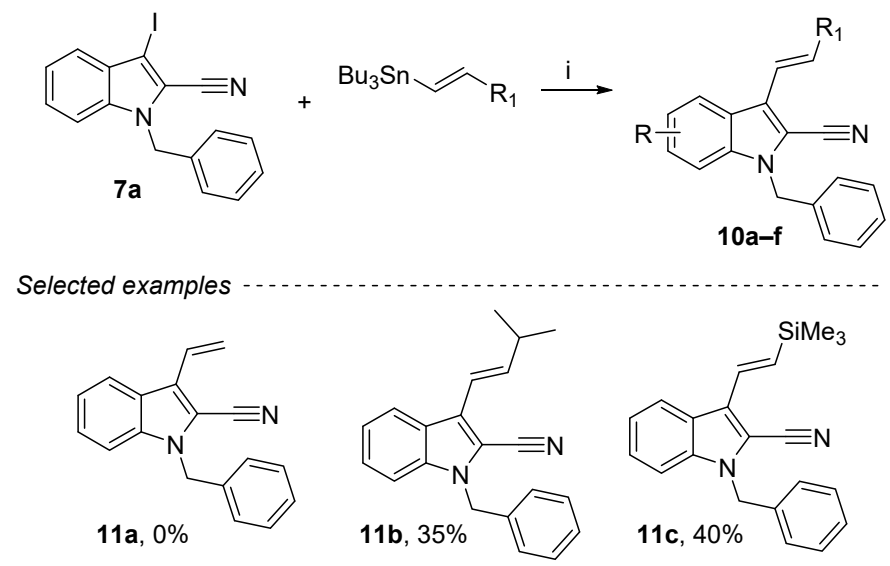

Scheme 8. Reagents and conditions: (i) $7 \mathrm{a}$ (1 equiv.), vinyltin derivatives (1.2 equiv.), $\mathrm{PdCl}_{2} \mathrm{MeCN}_{2}$ (10 mol\%), DMF, $40^{\circ} \mathrm{C}, 48 \mathrm{~h}$. 


\section{Materials and Methods}

\subsection{General Information}

The reagents were purchased from commercial suppliers and used without further purification. Melting points were determined on Büchi B-540 apparatus and are uncorrected. All solvents were dried following the procedure described by Armarego et Chai [57]. ${ }^{1} \mathrm{H}$ NMR and ${ }^{13} \mathrm{C}$ NMR spectra (from supplementary) were recorded on a Bruker Avance $300 \mathrm{MHz}$ at 300 and $75 \mathrm{MHz}$, respectively. ${ }^{1} \mathrm{H} \mathrm{NMR}$ spectra were recorded in $\mathrm{CDCl}_{3}$ or referenced the residual $\mathrm{CHCl}_{3}$ at $7.26 \mathrm{ppm}\left(2.50 \mathrm{ppm}\right.$ for DMSO- $\left.d_{6}\right) ;{ }^{13} \mathrm{C} \mathrm{NMR}$ and J-mod spectra were referenced to the central peak of $\mathrm{CDCl}_{3}$ at $77.0 \mathrm{ppm}\left(39.52 \mathrm{ppm}\right.$ for DMSO- $d_{6}$ ). ${ }^{19} \mathrm{~F} \mathrm{NMR}$ was recorded at $282 \mathrm{MHz}$ on the same instrument, using the $\mathrm{CFCl}_{3}$ as internal reference $(\delta 0.0)$. Chemical shifts were reported in parts per million $(\mathrm{ppm}, \delta)$, and coupling constants $(J)$ were given in Hertz $(\mathrm{Hz})$. Abbreviations for signal coupling are as follows: $\mathrm{s}$, singlet; $d$, doublet; $t$, triplet; $q$, quartet; quin, quintet; sextuplet; sext, dd, doublet of doublets; dq, doublet of quartets; m, multiplet. High-resolution mass spectra (HRMS) were obtained by the electrospray ionization time-of-flight (ESI) mass spectrometry. Thin-layer chromatography (TLC) was performed on TLC silica gel $60 \mathrm{~F}_{254}$. Compounds were visualized under UV light $(\lambda=254 \mathrm{~nm})$ and/or by immersion in a $\mathrm{KMnO}_{4}$ solution followed by heating. Products were purified by flash column chromatography on silica gel $(0.04-0.063 \mathrm{~mm})$ using various mixtures of EtOAc and petroleum ether $\left(35-60^{\circ} \mathrm{C}\right.$ fraction) as eluent. Heating was performed using a magnetic stirrer hotplate and an appropriate-sized heating block. Tributyl(vinyl)stannane was prepared from vinylmagnesium bromide and bis(tributyltin) oxide [58,59]. (E)-1-(Tributylstannyl)-2-(trimethylsilyl)ethene was prepared by hydrostannation of (trimethylsilyl)acetylene [60]. (E)-Tributyl(3-methylbut-1-en-1-yl)stannane was prepared by the method described by Chong [61]. The compound's name follows the IUPAC recommendations.

\subsection{Experimental Section}

3.2.1. General Procedure for the Synthesis of Nitriles (Series 3)

To a solution of amide ( $4.0 \mathrm{~g}, 25.0 \mathrm{mmol}, 1.0$ equiv.) in chloroform $(75 \mathrm{~mL})$ was added dropwise phosphorus oxychloride $(9.24 \mathrm{~mL}, 99.1 \mathrm{mmol}, 4.0$ equiv.). The mixture was stirred under reflux for $3 \mathrm{~h}$ and then cooled to room temperature. The reaction mixture was quenched by a $25 \% \mathrm{NH}_{4} \mathrm{OH}$ aq. Solution $(20 \mathrm{~mL})$ and the resulting aqueous layer were extracted with $\mathrm{Et}_{2} \mathrm{O}(3 \times 30 \mathrm{~mL})$. The organic layers were combined, dried over $\mathrm{MgSO}_{4}$ and concentrated under reduced pressure. The crude product was purified by column chromatography using petroleum ether/ethyl acetate (80:20) as eluent.

1H-Indole-2-carbonitrile (3a): Yellow solid, 79\%, 2.83 g. ${ }^{1} \mathrm{H} \mathrm{NMR}\left(300 \mathrm{MHz}, \mathrm{CDCl}_{3}\right)$ : $\delta=8.66(\mathrm{bs}, 1 \mathrm{H}, \mathrm{NH}), 7.68(\mathrm{dq}, J=8.1 \mathrm{~Hz}, 0.9 \mathrm{~Hz}, 1 \mathrm{H}, \mathrm{Ar}-\mathrm{H}), 7.45-7.36(\mathrm{~m}, 2 \mathrm{H}, \mathrm{Ar}-\mathrm{H}), 7.25-$ 7.19 (m, 2H, Ar-H) ppm. ${ }^{13} \mathrm{C}$ NMR (75 MHz, $\left.\mathrm{CDCl}_{3}\right): \delta=137.1$ (C quat $), 126.3(\mathrm{Ar}-\mathrm{CH}), 126.2$ ( $\left.\mathrm{C}_{\text {quat }}\right), 122.1(\mathrm{Ar}-\mathrm{CH}), 121.7(\mathrm{Ar}-\mathrm{CH}), 114.6\left(\mathrm{C}_{\text {quat }}\right), 114.5(\mathrm{Ar}-\mathrm{CH}), 112.0(\mathrm{Ar}-\mathrm{CH}), 106.0$ $\left(\mathrm{C}_{\text {quat }}\right) \mathrm{ppm}$. These spectroscopic data correspond to the reported data in reference [28].

5-Methoxy-1H-indole-2-carbonitrile (3b): Yellow solid, 77\%, 2.85 g. ${ }^{1} \mathrm{H}$ NMR $(300 \mathrm{MHz}$, $\left.\mathrm{CDCl}_{3}\right): \delta=8.57(\mathrm{bs}, 1 \mathrm{H}, \mathrm{NH}), 7.31(\mathrm{dd}, J=9.7 \mathrm{~Hz}, J=0.9 \mathrm{~Hz}, 1 \mathrm{H}, \mathrm{Ar}-H), 7.12(\mathrm{dd}$, $J=2.1 \mathrm{~Hz}, J=0.9 \mathrm{~Hz}, 1 \mathrm{H}, \mathrm{Ar}-H), 7.07(\mathrm{~d}, J=2.4 \mathrm{~Hz}, 1 \mathrm{H}, \mathrm{Ar}-\mathrm{H}), 7.06-7.03(\mathrm{~m}, 1 \mathrm{H}, \mathrm{Ar}-\mathrm{H})$, $6.83(\mathrm{~d}, J=1.3 \mathrm{~Hz}, 1 \mathrm{H}, \mathrm{Ar}-\mathrm{H}), 3.85\left(\mathrm{~s}, 3 \mathrm{H}, \mathrm{OCH}_{3}\right) \mathrm{ppm} .{ }^{13} \mathrm{C}$ NMR $\left(75 \mathrm{MHz}, \mathrm{CDCl}_{3}\right)$ : $\delta=155.5\left(\mathrm{C}_{\text {quat }}\right), 132.3\left(\mathrm{C}_{\text {quat }}\right), 126.9\left(\mathrm{C}_{\text {quat }}\right), 118.1(\mathrm{Ar}-\mathrm{CH}), 114.4\left(\mathrm{C}_{\text {quat }}\right), 114.0(\mathrm{Ar}-\mathrm{CH})$, 112.7 (Ar- $\mathrm{CH}), 106.6\left(\mathrm{C}_{\text {quat }}\right), 102.1(\mathrm{Ar}-\mathrm{CH}), 55.8\left(\mathrm{OCH}_{3}\right) \mathrm{ppm}$. These spectroscopic data correspond to the reported data in reference [45].

6-Methoxy-1H-indole-2-carbonitrile (3c): Yellow solid, 77\%, $2.85 \mathrm{~g} .{ }^{1} \mathrm{H}$ NMR $(300 \mathrm{MHz}$, $\left.\mathrm{CDCl}_{3}\right): \delta=8.71(\mathrm{bs}, 1 \mathrm{H}, \mathrm{NH}), 7.52(\mathrm{~d}, J=8.8 \mathrm{~Hz}, 1 \mathrm{H}, \mathrm{Ar}-H), 7.13(\mathrm{~d}, J=1.3 \mathrm{~Hz}, 1 \mathrm{H}, \mathrm{Ar}-H)$, $6.88(\mathrm{dd}, J=8,8 \mathrm{~Hz}, 2.1 \mathrm{~Hz}, 1 \mathrm{H}, \mathrm{Ar}-H), 6.83(\mathrm{~d}, J=1.3 \mathrm{~Hz}, 1 \mathrm{H}, \mathrm{Ar}-\mathrm{H}), 3.86\left(\mathrm{~s}, 3 \mathrm{H}, \mathrm{OCH}_{3}\right)$ ppm. ${ }^{13} \mathrm{C}$ NMR $\left(75 \mathrm{MHz}, \mathrm{CDCl}_{3}\right): \delta=159.7\left(\mathrm{C}_{\text {quat }}\right), 138.2\left(\mathrm{C}_{\text {quat }}\right), 122.9(\mathrm{Ar}-\mathrm{CH}), 120.6$ $\left(\mathrm{C}_{\text {quat }}\right), 114.9(\mathrm{Ar}-\mathrm{CH}), 114.8\left(\mathrm{C}_{\text {quat }}\right), 113.4(\mathrm{Ar}-\mathrm{CH}), 104.8\left(\mathrm{C}_{\text {quat }}\right), 93.8(\mathrm{CH}), 55.7\left(\mathrm{OCH}_{3}\right)$ $\mathrm{ppm}$. These spectroscopic data correspond to the reported data in reference [27]. 
5-Fluoro-1H-indole-2-carbonitrile (3d): Yellow solid, 81\%, 2.90 g. ${ }^{1} \mathrm{H}$ NMR $(300 \mathrm{MHz}$, $\left.\mathrm{CDCl}_{3}\right): \delta=8.64(\mathrm{bs}, 1 \mathrm{H}, \mathrm{NH}), 7.37(\mathrm{dd}, J=9.0 \mathrm{~Hz}, 4.3 \mathrm{~Hz}, 1 \mathrm{H}, \mathrm{Ar}-H), 7.32(\mathrm{dd}$, $J=8.9 \mathrm{~Hz}, 2.5 \mathrm{~Hz}, 1 \mathrm{H}, \mathrm{Ar}-\mathrm{H}), 7.20-7.12(\mathrm{~m}, 2 \mathrm{H}, \mathrm{Ar}-\mathrm{CH}) \mathrm{ppm} .{ }^{13} \mathrm{C} \mathrm{NMR}\left(75 \mathrm{MHz}, \mathrm{CDCl}_{3}\right)$ : $\delta=158.7\left(\mathrm{~d}, J=238.6 \mathrm{~Hz}, \mathrm{C}_{\text {quat }}\right), 133.6\left(\mathrm{C}_{\text {quat }}\right), 126.6\left(\mathrm{~d}, J=10.6 \mathrm{~Hz}, \mathrm{C}_{\text {quat }}\right), 115.7(\mathrm{~d}$, $J=27.1 \mathrm{~Hz}, \mathrm{Ar}-\mathrm{CH}), 114.3(\mathrm{~d}, J=5.4 \mathrm{~Hz}, \mathrm{Ar}-\mathrm{CH}), 114.1\left(\mathrm{C}_{\text {quat }}\right), 113.0(\mathrm{~d}, J=9.5 \mathrm{~Hz}$, Ar- $\mathrm{CH}), 107.7\left(\mathrm{C}_{\text {quat }}\right), 106.5$ (d, $\left.J=23.8 \mathrm{~Hz}, \mathrm{Ar}-\mathrm{CH}\right) \mathrm{ppm} .{ }^{19} \mathrm{~F}$ NMR $\left(282 \mathrm{MHz}, \mathrm{CDCl}_{3}\right)$ : $\delta=-119.9 \mathrm{ppm}$. These spectroscopic data correspond to the reported data in reference [45].

\subsubsection{Typical Procedure for the Synthesis of Propargyl Compound 4}

In a two-neck round bottom flask, $\mathrm{NaH} 60 \%(0.60 \mathrm{~g}, 15.0 \mathrm{mmol}, 1.3$ equiv. $)$ was dissolved in DMF $(15 \mathrm{~mL})$, and indole $(7.03 \mathrm{mmol}, 1$ equiv.) in DMF $(10 \mathrm{~mL})$ was added dropwise at $0{ }^{\circ} \mathrm{C}$ under argon. The mixture was stirred for $30 \mathrm{~min}$ at room temperature. Propargyl bromide $80 \%$ (11.4 mmol, 1.3 equiv.) was diluted in DMF and added dropwise into the flask at $0{ }^{\circ} \mathrm{C}$ under argon. After $3-4 \mathrm{~h}$ of stirring at room temperature, the mixture was hydrolyzed with sat. aq. $\mathrm{NH}_{4} \mathrm{Cl}(10 \mathrm{~mL})$, extracted with $\mathrm{Et}_{2} \mathrm{O}(7 \times 20 \mathrm{~mL})$, and the combined organic layers were washed with brine $(6 \times 10 \mathrm{~mL})$, dried over $\mathrm{MgSO}_{4}$ and evaporated under reduced pressure.

1-(Prop-2-yn-1-yl)-1H-indole-2-carbonitrile (4a): Yellow solid, 75\%, 0.750 g. ${ }^{1} \mathrm{H}$ NMR $\left(300 \mathrm{MHz}, \mathrm{CDCl}_{3}\right): \delta=7.69(\mathrm{dt}, J=8.1 \mathrm{~Hz}, 1 \mathrm{H}, \mathrm{Ar}-H), 7.51(\mathrm{dd}, J=8.4 \mathrm{~Hz}, J=0.9 \mathrm{~Hz}, 1 \mathrm{H}$, Ar- $H), 7.48-7.41(\mathrm{~m}, 1 \mathrm{H}, \mathrm{Ar}-\mathrm{H}), 7.29-7.19(\mathrm{~m}, 2 \mathrm{H}, \mathrm{Ar}-\mathrm{H}), 5.04\left(\mathrm{~s}, 2 \mathrm{H}, \mathrm{CH}_{2}\right), 2.40(\mathrm{t}, J=2.5 \mathrm{~Hz}$, $1 \mathrm{H}, \mathrm{CH}) \mathrm{ppm} .{ }^{13} \mathrm{C} \mathrm{NMR}\left(75 \mathrm{MHz}, \mathrm{CDCl}_{3}\right): \delta=137.3\left(\mathrm{C}_{\text {quat }}\right), 126.6\left(\mathrm{C}_{\text {quat }}\right), 126.4(\mathrm{Ar}-\mathrm{CH})$, 122.7 (Ar-CH), $122.0(\mathrm{Ar}-\mathrm{CH}), 114.2(\mathrm{Ar}-\mathrm{CH}), 113.2\left(\mathrm{C}_{\text {quat }}\right), 110.7(\mathrm{Ar}-\mathrm{CH}), 109.6\left(\mathrm{C}_{\text {quat }}\right), 76.5$ $\left(\mathrm{C}_{\text {quat }}\right), 74.2(\mathrm{CH}), 34.4\left(\mathrm{CH}_{2}\right) \mathrm{ppm}$. These spectroscopic data correspond to the reported data in reference [10].

\subsubsection{General Procedure for the Synthesis of Nitriles (Series 5)}

A dried Schlenk tube was charged with compound $4 \mathrm{a}$ ( $1.6 \mathrm{mmol}, 1$ equiv.), aryl iodide derivative $\left(2.1 \mathrm{mmol}, 1.3\right.$ equiv.) and DMF $(5 \mathrm{~mL})$. The solution was cooled to $0{ }^{\circ} \mathrm{C}$ for 15 min under argon ant $\mathrm{Et}_{3} \mathrm{~N}$ (4.8 mmol, 3 equiv.) was added into the Schlenk. CuI $(0.16 \mathrm{mmol}, 10 \mathrm{~mol} \%)$ and $\mathrm{PdCl}_{2}\left(\mathrm{PPh}_{3}\right)_{2}(0.16 \mathrm{mmol}, 10 \mathrm{~mol} \%)$ were added, and the mixture was stirred overnight at room temperature. The mixture was hydrolyzed with sat. aq. $\mathrm{NH}_{4} \mathrm{Cl}(10 \mathrm{~mL})$ and extracted with $\mathrm{Et}_{2} \mathrm{O}(7 \times 20 \mathrm{~mL})$. The organic layers were washed with brine $(2 \times 10 \mathrm{~mL})$, dried over $\mathrm{MgSO}_{4}$ and evaporated under reduced pressure.

1-(3-(p-Tolyl)prop-2-yn-1-yl)-1H-indole-2-carbonitrile (5a): Yellow solid, 70\%, $252 \mathrm{mg} . \mathrm{mp}$ $96-98{ }^{\circ} \mathrm{C} .{ }^{1} \mathrm{H}$ NMR $\left(300 \mathrm{MHz}, \mathrm{CDCl}_{3}\right): \delta=7.69(\mathrm{~d}, J=8.1 \mathrm{~Hz}, 1 \mathrm{H}, \mathrm{Ar}-H), 7.59(\mathrm{dd}, J=8.5 \mathrm{~Hz}$, $8.0 \mathrm{~Hz}, 1 \mathrm{H}, \mathrm{Ar}-H), 7.45(\mathrm{ddd}, J=8.4 \mathrm{~Hz}, J=7.0 \mathrm{~Hz}, J=1.1 \mathrm{~Hz}, 1 \mathrm{H}, \mathrm{Ar}-H), 7.29(\mathrm{~d}, J=8.1 \mathrm{~Hz}$, $2 \mathrm{H}), 7.27-7.21(\mathrm{~m}, 2 \mathrm{H}), 7.09(\mathrm{~d}, J=7.9 \mathrm{~Hz}, 2 \mathrm{H}), 5.25\left(\mathrm{~s}, 2 \mathrm{H}, \mathrm{CH}_{2}\right), 2.32\left(\mathrm{~s}, 3 \mathrm{H}, \mathrm{CH}_{3}\right) \mathrm{ppm}$. ${ }^{13} \mathrm{C}$ NMR $\left(75 \mathrm{MHz}, \mathrm{CDCl}_{3}\right): \delta=139.1\left(\mathrm{C}_{\text {quat }}\right), 137.3\left(\mathrm{C}_{\text {quat }}\right), 131.8(2 \mathrm{Ar}-\mathrm{CH}), 129.1(2 \mathrm{Ar}-\mathrm{CH})$, 126.5 ( $\left.\mathrm{C}_{\text {quat }}\right), 126.2(\mathrm{Ar}-\mathrm{CH}), 122.5(\mathrm{Ar}-\mathrm{CH}), 121.8(\mathrm{Ar}-\mathrm{CH}), 118.8\left(\mathrm{C}_{\text {quat }}\right), 113.9(\mathrm{Ar}-\mathrm{CH})$, $113.4\left(\mathrm{C}_{\text {quat }}\right), 110.8(\mathrm{Ar}-\mathrm{CH}), 109.5\left(\mathrm{C}_{\text {quat }}\right), 85.9\left(\mathrm{C}_{\text {quat }}\right), 81.2\left(\mathrm{C}_{\text {quat }}\right), 35.7\left(\mathrm{CH}_{2}\right), 21.5\left(\mathrm{CH}_{3}\right)$ ppm. HRMS (ESI): calcd. for $\mathrm{C}_{19} \mathrm{H}_{15} \mathrm{~N}_{2}(\mathrm{M}+\mathrm{H})^{+} 271.12352$; found 271.12347.

1-(3-(4-Chlorophenyl)prop-2-yn-1-yl)-1H-indole-2-carbonitrile (5b): Yellow solid, 90\%, 353 $\mathrm{mg}) . \mathrm{mp} 98-100{ }^{\circ} \mathrm{C} .{ }^{1} \mathrm{H}$ NMR $\left(300 \mathrm{MHz}, \mathrm{CDCl}_{3}\right): \delta=7.69(\mathrm{~d}, J=8.1 \mathrm{~Hz}, 1 \mathrm{H}, \mathrm{Ar}-\mathrm{H}), 7.56$ $(\mathrm{d}, J=8.4 \mathrm{~Hz}, 1 \mathrm{H}, \mathrm{Ar}-\mathrm{H}), 7.46(\mathrm{ddd}, J=8.1 \mathrm{~Hz}, J=7.0 \mathrm{~Hz}, J=1.0 \mathrm{~Hz}, 1 \mathrm{H}, \mathrm{Ar}-\mathrm{H}), 7.33(\mathrm{~d}, J$ $=8.6 \mathrm{~Hz}, 2 \mathrm{H}, \mathrm{Ar}-\mathrm{H}), 7.30-7.22(\mathrm{~m}, 4 \mathrm{H}, \mathrm{Ar}-\mathrm{H}), 5.25\left(\mathrm{~s}, 2 \mathrm{H}, \mathrm{CH}_{2}\right) \mathrm{ppm} .{ }^{13} \mathrm{C} \mathrm{NMR}(75 \mathrm{MHz}$, $\left.\mathrm{CDCl}_{3}\right): \delta=137.3\left(\mathrm{C}_{\text {quat }}\right), 135.1\left(\mathrm{C}_{\text {quat }}\right), 133.2(2 \mathrm{Ar}-\mathrm{CH}), 128.8(2 \mathrm{Ar}-\mathrm{CH}), 126.6\left(\mathrm{C}_{\text {quat }}\right), 126.4$ (Ar-CH), $122.7(\mathrm{Ar}-\mathrm{CH}), 122.0(\mathrm{Ar}-\mathrm{CH}), 120.4\left(\mathrm{C}_{\text {quat }}\right), 114.2(\mathrm{Ar}-\mathrm{CH}), 113.3\left(\mathrm{C}_{\text {quat }}\right), 110.7$ (Ar- $\mathrm{CH}), 109.6\left(\mathrm{C}_{\text {quat }}\right), 84.7\left(\mathrm{C}_{\text {quat }}\right), 82.9\left(\mathrm{C}_{\text {quat }}\right), 35.6\left(\mathrm{CH}_{2}\right) \mathrm{ppm}$. HRMS (ESI): calcd. for $\mathrm{C}_{18} \mathrm{H}_{12} \mathrm{ClN}_{2}(\mathrm{M}+\mathrm{H})^{+}$291.06890; found 291.06901.

1-(3-(4-(Trifluoromethyl)phenyl)prop-2-yn-1-yl)-1H-indole-2-carbonitrile (5c): Yellow solid, 78\%, $341 \mathrm{mg}$. mp 110-112 ${ }^{\circ} \mathrm{C} .{ }^{1} \mathrm{H} \mathrm{NMR}\left(300 \mathrm{MHz}, \mathrm{CDCl}_{3}\right): \delta=7.71(\mathrm{~d}, J=8.1 \mathrm{~Hz}, 1 \mathrm{H}, \mathrm{Ar}-\mathrm{H})$, 7.60-7.53 (m, 3H, Ar-H), 7.53-7.42 (m, 3H, Ar-H), $7.29(\mathrm{~m}, 2 \mathrm{H}), 7.29(\mathrm{dd}, J=7.1 \mathrm{~Hz}, J=0.9$ $\mathrm{Hz}, 1 \mathrm{H}, \mathrm{Ar}-\mathrm{H}), 7.24(\mathrm{~s}, 1 \mathrm{H}, \mathrm{Ar}-\mathrm{H}), 5.28\left(\mathrm{~s}, 2 \mathrm{H}, \mathrm{CH}_{2}\right) \mathrm{ppm} .{ }^{13} \mathrm{C} \mathrm{NMR}\left(75 \mathrm{MHz}, \mathrm{CDCl}_{3}\right): \delta=$ $137.4\left(\mathrm{C}_{\text {quat }}\right), 132.3(2 \mathrm{Ar}-\mathrm{CH}), 130.7\left(\mathrm{q}, J=32.5 \mathrm{~Hz}, \mathrm{C}_{\text {quat }}\right), 126.6\left(\mathrm{C}_{\text {quat }}\right), 125.7(\mathrm{q}, J=1.3 \mathrm{~Hz}$, 
$\left.\mathrm{C}_{\text {quat }}\right), 125.4$ (q, $\left.J=3.7 \mathrm{~Hz}, 2 \mathrm{Ar}-\mathrm{CH}\right), 123.9$ (q, $\left.J=272.8 \mathrm{~Hz}, \mathrm{C}_{\text {quat }}\right), 122.7(\mathrm{Ar}-\mathrm{CH}), 122.1$ $(\mathrm{Ar}-\mathrm{CH}), 114.3(\mathrm{Ar}-\mathrm{CH}), 113.3\left(\mathrm{C}_{\text {quat }}\right), 110.6(\mathrm{Ar}-\mathrm{CH}), 109.6\left(\mathrm{C}_{\text {quat }}\right), 84.4\left(\mathrm{C}_{\text {quat }}\right), 84.3\left(\mathrm{C}_{\text {quat }}\right)$, $35.6\left(\mathrm{CH}_{2}\right)$ ppm. HRMS (ESI): calcd. for $\mathrm{C}_{19} \mathrm{H}_{12} \mathrm{~F}_{3} \mathrm{~N}_{2}(\mathrm{M}+\mathrm{H})^{+} 325.09471$; found 325.09395 . 1-(3-(2-Cyanophenyl)prop-2-yn-1-yl)-1H-indole-2-carbonitrile (5d): Yellow solid, 64\%, $379 \mathrm{mg}$. mp 137-139 ${ }^{\circ} \mathrm{C} .{ }^{1} \mathrm{H}$ NMR $\left(300 \mathrm{MHz}, \mathrm{CDCl}_{3}\right): \delta=7.71-7.62(\mathrm{~m}, 2 \mathrm{H}, \mathrm{Ar}-\mathrm{H}), 7.54-7.47$ (m, 3H, Ar-H), 7.46-7.39 (m, 2H, Ar-H), 7.29-7.22 (m, 2H, Ar-H), 5.34 (s, 2H, CH $)$ ppm. ${ }^{13} \mathrm{C} \mathrm{NMR}\left(75 \mathrm{MHz}, \mathrm{CDCl}_{3}\right): \delta=137.4\left(\mathrm{C}_{\text {quat }}\right), 132.9(\mathrm{Ar}-\mathrm{CH}), 132.7(\mathrm{Ar}-\mathrm{CH}), 132.5(\mathrm{Ar}-\mathrm{CH})$, 129.2 (Ar-CH), $126.63(\mathrm{Ar}-\mathrm{CH}), 126.6\left(\mathrm{C}_{\text {quat }}\right), 125.8\left(\mathrm{C}_{\text {quat }}\right), 122.6(\mathrm{Ar}-\mathrm{CH}), 122.1(\mathrm{Ar}-\mathrm{CH})$, 117.3 ( $\left.\mathrm{C}_{\text {quat }}\right), 115.7$ ( $\left.\mathrm{C}_{\text {quat }}\right), 114.4(\mathrm{Ar}-\mathrm{CH}), 113.3$ ( $\left.\mathrm{C}_{\text {quat }}\right), 111.0(\mathrm{Ar}-\mathrm{CH}), 109.5$ ( $\left.\mathrm{C}_{\text {quat }}\right), 88.3$ ( $\left.\mathrm{C}_{\text {quat }}\right), 81.9$ ( $\left.\mathrm{C}_{\text {quat }}\right), 35.6\left(\mathrm{CH}_{2}\right)$ ppm. HRMS (ESI): calcd. for $\mathrm{C}_{19} \mathrm{H}_{11} \mathrm{~N}_{3}(\mathrm{M}+\mathrm{H})^{+}$282.10257; found 282.10179.

1-(3-(2-Methoxyphenyl)prop-2-yn-1-yl)-1H-indole-2-carbonitrile (5e): Yellow solid, 71\%, 269 mg. mp 96-98 ${ }^{\circ} \mathrm{C} .{ }^{1} \mathrm{H}$ NMR $\left(300 \mathrm{MHz}, \mathrm{CDCl}_{3}\right): \delta=7.70-7.64(\mathrm{~m}, 2 \mathrm{H}, \mathrm{Ar}-\mathrm{H}), 7.45$ (ddd, $J=8.5 \mathrm{~Hz}, J=7.1 \mathrm{~Hz}, J=1.1 \mathrm{~Hz}, 1 \mathrm{H}, \mathrm{Ar}-H), 7.35(\mathrm{dd}, J=7.5 \mathrm{~Hz}, J=1.6 \mathrm{~Hz}, 1 \mathrm{H}, \mathrm{Ar}-H)$, 7.32-7.19 (m, 3H, 3Ar-H), $6.87(\mathrm{td}, J=7.5 \mathrm{~Hz}, J=0.9 \mathrm{~Hz}, 1 \mathrm{H}, \mathrm{Ar}-H), 6.84(\mathrm{~d}, J=8.4 \mathrm{~Hz}$, $\mathrm{Ar}-\mathrm{H}), 5.29\left(\mathrm{~s}, 2 \mathrm{H}, \mathrm{CH}_{2}\right), 3.84\left(\mathrm{~s}, \mathrm{OCH}_{3}\right) \mathrm{ppm} .{ }^{13} \mathrm{C} \mathrm{NMR}\left(75 \mathrm{MHz}, \mathrm{CDCl}_{3}\right): \delta=160.4\left(\mathrm{C}_{\text {quat }}\right)$, 137.4 ( $\left.\mathrm{C}_{\text {quat }}\right), 133.9$ (Ar-CH), $130.4(\mathrm{Ar}-\mathrm{CH}), 126.6$ ( $\left.\mathrm{C}_{\text {quat }}\right), 126.1(\mathrm{Ar}-\mathrm{CH}), 122.5(\mathrm{Ar}-\mathrm{CH})$, 121.8 (Ar-CH), 120.5 (Ar-CH), $113.9(\mathrm{Ar}-\mathrm{CH}), 113.4$ (C $\left.\mathrm{C}_{\text {quat }}\right), 111.2\left(2 \mathrm{Ar}-\mathrm{CH}+\mathrm{C}_{\text {quat }}\right), 110.8$ $(\mathrm{Ar}-\mathrm{CH}), 109.6\left(\mathrm{C}_{\text {quat }}\right), 85.8\left(\mathrm{C}_{\text {quat }}\right), 82.4\left(\mathrm{C}_{\text {quat }}\right), 55.8\left(\mathrm{OCH}_{3}\right), 36.1\left(\mathrm{CH}_{2}\right) \mathrm{ppm}$. HRMS (ESI): calcd. for $\mathrm{C}_{19} \mathrm{H}_{15} \mathrm{~N}_{2} \mathrm{O}(\mathrm{M}+\mathrm{H})^{+} 287.11844$; found 287.11835 .

1-(3-(3,4-Dichlorophenyl)prop-2-yn-1-yl)-1H-indole-2-carbonitrile (5f): Yellow solid, 87\%, $381 \mathrm{mg} . \mathrm{mp} 115-117^{\circ} \mathrm{C} .{ }^{1} \mathrm{H}$ NMR $\left(300 \mathrm{MHz} \mathrm{CDCl}_{3}\right): \delta=7.70(\mathrm{~d}, J=8.1 \mathrm{~Hz}, 1 \mathrm{H}, \mathrm{Ar}-\mathrm{H})$, $7.54(\mathrm{~d}, J=8.4 \mathrm{~Hz}, 1 \mathrm{H}, \mathrm{Ar}-H), 7.50-7.44(\mathrm{~m}, 2 \mathrm{H}, \mathrm{Ar}-H), 7.36(\mathrm{~d}, J=8.3 \mathrm{~Hz}, 1 \mathrm{H}, \mathrm{Ar}-H), 7.27$ $(\mathrm{dd}, J=7.0 \mathrm{~Hz}, J=1.0 \mathrm{~Hz}, 1 \mathrm{H}, \mathrm{Ar}-H), 7.23(\mathrm{~s}, 1 \mathrm{H}, \mathrm{Ar}-H), 7.20(\mathrm{dd}, J=8.4 \mathrm{~Hz}, J=1.9 \mathrm{~Hz}, 1 \mathrm{H}$, $\mathrm{Ar}-\mathrm{H}), 5.25\left(\mathrm{~s}, 2 \mathrm{H}, \mathrm{CH}_{2}\right)$ ppm. ${ }^{13} \mathrm{C} \mathrm{NMR}\left(75 \mathrm{MHz}, \mathrm{CDCl}_{3}\right): \delta=137.3\left(\mathrm{C}_{\text {quat }}\right), 133.5(\mathrm{Ar}-\mathrm{CH})$, 132.7 (C quat $), 131.1(\mathrm{Ar}-\mathrm{CH}), 130.5(\mathrm{Ar}-\mathrm{CH}), 126.6\left(\mathrm{C}_{\text {quat }}\right), 126.5(\mathrm{Ar}-\mathrm{CH}), 122.7(\mathrm{Ar}-\mathrm{CH})$, $122.1(\mathrm{Ar}-\mathrm{CH}), 121.8$ ( $\left.\mathrm{C}_{\text {quat }}\right), 114.3(\mathrm{Ar}-\mathrm{CH}), 113.3$ ( $\left.\mathrm{C}_{\text {quat }}\right), 111.8$ (C $\left.\mathrm{C}_{\text {quat }}\right), 110.5(\mathrm{Ar}-\mathrm{CH}), 109.6$ $\left(\mathrm{C}_{\text {quat }}\right), 83.9$ (C $\left.\mathrm{C}_{\text {quat }}\right), 83.5\left(\mathrm{C}_{\text {quat }}\right), 35.5\left(\mathrm{CH}_{2}\right)$ ppm. HRMS (ESI): calcd. for $\mathrm{C}_{18} \mathrm{H}_{10}{ }^{35} \mathrm{Cl}_{2} \mathrm{~N}_{2}$ $(\mathrm{M}+\mathrm{H})^{+}$325.02938; found 325.02841 .

\subsubsection{General Procedure for the Synthesis of Nitriles (Series 6)}

To a solution of $1 H$-indole-2-carbonitrile ( $2.0 \mathrm{~g}, 14.1 \mathrm{mmol}, 1.0$ equiv.) in DMF (10 mL), $\mathrm{KOH}(0.79 \mathrm{~g}, 50.3 \mathrm{mmol}, 3.6$ equiv.) was added in small portions. The mixture was stirred for $30 \mathrm{~min}$ at room temperature. Then, a solution of iodine ( $3.57 \mathrm{~g}, 14.1 \mathrm{mmol}, 1.0 \mathrm{equiv}$.) in DMF ( $3 \mathrm{~mL}$ ) was added dropwise at $0{ }^{\circ} \mathrm{C}$, and the mixture was stirred for $4 \mathrm{~h}$ at room temperature. The mixture was then poured into a mixture of water $(600 \mathrm{~mL})$ and sat. aq. $\mathrm{NH}_{4} \mathrm{Cl}(40 \mathrm{~mL})$ and stirred for $30 \mathrm{~min}$. The precipitate was filtered on a Büchner funnel and dried under vacuum for $2 \mathrm{~h}$.

3-Iodo-1H-indole-2-carbonitrile (6a): White solid, 77\%, $2.90 \mathrm{~g} .{ }^{1} \mathrm{H} \mathrm{NMR}(300 \mathrm{MHz}$, $\left.\mathrm{CDCl}_{3}\right): \delta=8.98(\mathrm{bs}, 1 \mathrm{H}, \mathrm{NH}), 7.49(\mathrm{dq}, J=8.1 \mathrm{~Hz}, J=0.9 \mathrm{~Hz}, 1 \mathrm{H}, \mathrm{Ar}-H), 7.47-7.39(\mathrm{~m}, 2 \mathrm{H}$, Ar- $H), 7.30(\mathrm{ddd}, J=8.1 \mathrm{~Hz}, J=6.2 \mathrm{~Hz}, J=1.8 \mathrm{~Hz}, 1 \mathrm{H}, \mathrm{Ar}-H)$ ppm. ${ }^{13} \mathrm{C}$ NMR $(75 \mathrm{MHz}$, DMSO-d 6$): \delta=137.0$ ( $\left.\mathrm{C}_{\text {quat }}\right), 128.9$ (C quat $), 126.6(\mathrm{Ar}-\mathrm{CH}), 122.0(\mathrm{Ar}-\mathrm{CH}), 121.8(\mathrm{Ar}-\mathrm{CH})$, 114.3 ( $\left.\mathrm{C}_{\text {quat }}\right), 112.8(\mathrm{Ar}-\mathrm{CH}), 111.4\left(\mathrm{C}_{\text {quat }}\right), 72.0\left(\mathrm{C}_{\text {quat }}\right) \mathrm{ppm}$. These spectroscopic data correspond to the reported data in reference [46].

3-Iodo-5-methoxy-1H-indole-2-carbonitrile (6b): White solid, 78\%, $2.70 \mathrm{~g} .{ }^{1} \mathrm{H} \mathrm{NMR}$ $\left(300 \mathrm{MHz}, \mathrm{DMSO}-\mathrm{d}_{6}\right): \delta=12.74$ (bs, $\left.1 \mathrm{H}, \mathrm{NH}\right), 7.40(\mathrm{dd}, J=9.0 \mathrm{~Hz}, J=0.4 \mathrm{~Hz}, 1 \mathrm{H}, \mathrm{Ar}-H)$, $7.05(\mathrm{dd}, J=9.0 \mathrm{~Hz}, 2.5 \mathrm{~Hz}, 1 \mathrm{H}, \mathrm{Ar}-\mathrm{H}), 6.77$ (d, $J=2.3 \mathrm{~Hz}, 1 \mathrm{H}, \mathrm{Ar}-\mathrm{H}), 3.82$ (s, 3H, OCH $\left.{ }_{3}\right)$ ppm. ${ }^{13} \mathrm{C}$ NMR (75 MHz, DMSO-d 6$): \delta=155.4$ (C quat $), 132.1$ (C quat $), 129.3$ (C $\left.\mathrm{C}_{\text {quat }}\right), 118.3$ (Ar-CH), $114.4\left(\mathrm{C}_{\text {quat }}\right), 114.0(\mathrm{Ar}-\mathrm{CH}), 111.2\left(\mathrm{C}_{\text {quat }}\right), 101.3(\mathrm{Ar}-\mathrm{CH}), 70.8\left(\mathrm{C}_{\text {quat }}\right), 55.4\left(\mathrm{OCH}_{3}\right)$ ppm. HRMS (ESI): calcd. for $\mathrm{C}_{10} \mathrm{H}_{8} \mathrm{IN}_{2} \mathrm{O}(\mathrm{M}+\mathrm{H})^{+}$298.96813; found 298.96711 .

3-Iodo-6-methoxy-1H-indole-2-carbonitrile (6c): White solid, 81\%, $2.80 \mathrm{~g} .{ }^{1} \mathrm{H} \mathrm{NMR}$ $\left(300 \mathrm{MHz} \mathrm{CDCl}_{3}\right): \delta=9.09$ (bs, $\left.1 \mathrm{H}, \mathrm{NH}\right), 7.31(\mathrm{~d}, J=9.2 \mathrm{~Hz}, 1 \mathrm{H}, \mathrm{Ar}-H), 6.92(\mathrm{dd}, J=$ $9.2 \mathrm{~Hz}, 2.1 \mathrm{~Hz}, 1 \mathrm{H}, \mathrm{Ar}-\mathrm{H}), 6.80(\mathrm{~d}, J=2.1 \mathrm{~Hz}, 1 \mathrm{H}, \mathrm{Ar}-\mathrm{H}), 3.87\left(\mathrm{~s}, 3 \mathrm{H}, \mathrm{OCH}_{3}\right) \mathrm{ppm} .{ }^{13} \mathrm{C}$ $\operatorname{NMR}\left(75 \mathrm{MHz}, \mathrm{CDCl}_{3}\right): \delta=160.6$ (C quat), 137.8 (C $\left.\mathrm{C}_{\text {quat }}\right), 124.0$ ( $\left.\mathrm{C}_{\text {quat }}\right), 123.6(\mathrm{Ar}-\mathrm{CH}), 114.4$ 
(Ar-CH), $114.4\left(\mathrm{C}_{\text {quat }}\right), 110.4\left(\mathrm{C}_{\text {quat }}\right), 93.8(\mathrm{Ar}-\mathrm{CH}), 72.5\left(\mathrm{C}_{\text {quat }}\right), 55.8\left(\mathrm{OCH}_{3}\right) \mathrm{ppm}$. HRMS (ESI): calcd. for $\mathrm{C}_{10} \mathrm{H}_{8} \mathrm{IN}_{2} \mathrm{O}(\mathrm{M}+\mathrm{H})^{+}$298.96813; found 298.96741 .

5-Fluoro-3-iodo-1H-indole-2-carbonitrile (6d): White solid, 73\%, 2.60 g. ${ }^{1} \mathrm{H}$ NMR $\left(300 \mathrm{MHz}, \mathrm{DMSO}_{\mathrm{d}}\right.$ ): $\delta=12.05(\mathrm{bs}, 1 \mathrm{H}, \mathrm{NH}), 8.03(\mathrm{ddd}, J=9.0 \mathrm{~Hz}, 4.3 \mathrm{~Hz}, 0.4 \mathrm{~Hz}$, $1 \mathrm{H}, \mathrm{Ar}-H), 7.69(\mathrm{td}, J=9.2 \mathrm{~Hz}, J=2.5 \mathrm{~Hz}, 1 \mathrm{H}, \mathrm{Ar}-H), 7.58(\mathrm{ddd}, J=9.0 \mathrm{~Hz}, 2.5 \mathrm{~Hz}, 0.4 \mathrm{~Hz}$, $1 \mathrm{H}, \mathrm{Ar}-\mathrm{H}) \mathrm{ppm} .{ }^{13} \mathrm{C}$ NMR $\left(75 \mathrm{MHz}\right.$, Acetone- $\left.\mathrm{d}_{6}\right): \delta=159.9\left(\mathrm{~d}, J=238.1 \mathrm{~Hz}, \mathrm{C}_{\text {quat }}\right), 134.6$ $\left(\mathrm{C}_{\text {quat }}\right), 130.6\left(\mathrm{~d}, J=10.7 \mathrm{~Hz}, \mathrm{C}_{\text {quat }}\right), 116.6(\mathrm{~d}, J=27.2 \mathrm{~Hz}, \mathrm{Ar}-\mathrm{CH}), 115.3(\mathrm{~d}, J=9.5 \mathrm{~Hz}$, Ar-CH), $114.7\left(\mathrm{C}_{\text {quat }}\right), 114.0\left(\mathrm{C}_{\text {quat }}\right), 107.1(\mathrm{~d}, J=24.9 \mathrm{~Hz}, \mathrm{Ar}-\mathrm{CH}), 70.1\left(\mathrm{~d}, J=5.6 \mathrm{~Hz}, \mathrm{C}_{\text {quat }}\right)$ ppm. ${ }^{19} \mathrm{~F}$ NMR $\left(282 \mathrm{MHz}, \mathrm{CDCl}_{3}\right): \delta=-119.6 \mathrm{~Hz}$. HRMS (ESI): calcd. for $\mathrm{C}_{9} \mathrm{H}_{5} \mathrm{FIN}_{2}(\mathrm{M}+$ $\mathrm{H})^{+}$286.94814; found 286.20350.

\subsubsection{General Procedure for the Synthesis of Nitriles (Series 7)}

To compound 6a (2.0 g, $7.4 \mathrm{mmol}, 1$ equiv.) in DMF (10 mL), NaH ( $0.17 \mathrm{~g}, 44 \mathrm{mmol}$, 1.2 equiv.) was added portion-wise at $0{ }^{\circ} \mathrm{C}$ under argon. The mixture was stirred for $30 \mathrm{~min}$ at room temperature. Then, benzyl bromide $(0.57 \mathrm{~mL}, 0.0048 \mathrm{~mol}, 1.3$ equiv.) was added dropwise at $0{ }^{\circ} \mathrm{C}$. The mixture was stirred at room temperature for $3-4 \mathrm{~h}$ and hydrolyzed with sat. aq. $\mathrm{NH}_{4} \mathrm{Cl}(10 \mathrm{~mL})$. The aqueous phase was extracted with $\mathrm{DCM}(3 \times$ $20 \mathrm{~mL}$ ), and the combined organic layers were dried over $\mathrm{MgSO}_{4}$ and concentrated under reduced pressure. The product was purified by column chromatography using petroleum ether/ethyl acetate (80:20) as eluent.

1-Benzyl-3-iodo-1H-indole-2-carbonitrile (7a): White solid, 71\%, 1.90 g. $\mathrm{mp} 144-146{ }^{\circ} \mathrm{C}$. ${ }^{1} \mathrm{H}$ NMR $\left(300 \mathrm{MHz}, \mathrm{CDCl}_{3}\right): \delta=7.50(\mathrm{dt}, J=8.1 \mathrm{~Hz}, 1.0 \mathrm{~Hz}, 1 \mathrm{H}, \mathrm{Ar}-H), 7.41-7.38(\mathrm{~m}, 1 \mathrm{H}$, Ar-H), 7.37-7.27 (m, 5H, Ar-H), $7.18(\mathrm{dd}, J=7.5 \mathrm{~Hz}, J=2.0 \mathrm{~Hz}, 2 \mathrm{H}, \mathrm{Ar}-H), 5.51(\mathrm{~s}, 2 \mathrm{H}$, $\left.\mathrm{CH}_{2}\right)$ ppm. ${ }^{13} \mathrm{C}$ NMR $\left(75 \mathrm{MHz}, \mathrm{CDCl}_{3}\right): \delta=137.4\left(\mathrm{C}_{\text {quat }}\right), 135.6\left(\mathrm{C}_{\text {quat }}\right), 129.8\left(\mathrm{C}_{\text {quat }}\right), 129.2$ (2Ar- $\mathrm{CH}), 128.5(\mathrm{Ar}-\mathrm{CH}), 127.2(\mathrm{Ar}-\mathrm{CH}), 127.0(2 \mathrm{Ar}-\mathrm{CH}), 123.2(\mathrm{Ar}-\mathrm{CH}), 122.6(\mathrm{Ar}-\mathrm{CH})$, $115.4\left(\mathrm{C}_{\text {quat }}\right), 113.4\left(\mathrm{C}_{\text {quat }}\right), 111.1(\mathrm{Ar}-\mathrm{CH}), 70.6\left(\mathrm{C}_{\text {quat }}\right), 50.1\left(\mathrm{CH}_{2}\right) \mathrm{ppm}$. HRMS (ESI): calcd. for $\mathrm{C}_{16} \mathrm{H}_{12} \mathrm{IN}_{2}(\mathrm{M}+\mathrm{H})^{+}$359.00397; found 359.00293.

1-Benzyl-3-iodo-5-methoxy-1H-indole-2-carbonitrile (7b): White solid, $81 \%, 2.10 \mathrm{~g} .{ }^{1} \mathrm{H}$ NMR $\left(300 \mathrm{MHz}, \mathrm{CDCl}_{3}\right): \delta=7.35-7.27(\mathrm{~m}, 3 \mathrm{H}, \mathrm{Ar}-H), 7.20(\mathrm{~d}, J=9.1 \mathrm{~Hz}, 1 \mathrm{H}, \mathrm{Ar}-H), 7.15$ $(\mathrm{dd}, J=7.5 \mathrm{~Hz}, J=2.4 \mathrm{~Hz}, 2 \mathrm{H}, \mathrm{Ar}-H), 7.04(\mathrm{dd}, J=9.1 \mathrm{~Hz}, J=2.4 \mathrm{~Hz}, 1 \mathrm{H}, \mathrm{Ar}-H), 6.82(\mathrm{~d}, J=$ $2.4 \mathrm{~Hz}, 1 \mathrm{H}, \mathrm{Ar}-\mathrm{H}), 5.47\left(\mathrm{~s}, 2 \mathrm{H}, \mathrm{CH}_{2}\right), 3.88\left(\mathrm{~s}, 3 \mathrm{H}, \mathrm{OCH}_{3}\right) \mathrm{ppm} .{ }^{13} \mathrm{C} \mathrm{NMR}\left(75 \mathrm{MHz}, \mathrm{CDCl}_{3}\right)$ : $\delta=156.3\left(\mathrm{C}_{\text {quat }}\right), 135.7\left(\mathrm{C}_{\text {quat }}\right), 132.7\left(\mathrm{C}_{\text {quat }}\right), 130.2\left(\mathrm{C}_{\text {quat }}\right), 129.2(2 \mathrm{Ar}-\mathrm{CH}), 128.4(\mathrm{Ar}-\mathrm{CH})$, $126.9(2 \mathrm{Ar}-\mathrm{CH}), 119.1(\mathrm{Ar}-\mathrm{CH}), 115.3\left(\mathrm{C}_{\text {quat }}\right), 113.5\left(\mathrm{C}_{\text {quat }}\right), 112.2(\mathrm{Ar}-\mathrm{CH}), 102.7(\mathrm{Ar}-\mathrm{CH})$, $69.4\left(\mathrm{C}_{\text {quat }}\right), 55.9\left(\mathrm{OCH}_{3}\right), 50.2\left(\mathrm{CH}_{2}\right)$ ppm. HRMS (ESI): calcd. for $\mathrm{C}_{17} \mathrm{H}_{14} \mathrm{IN}_{2} \mathrm{O}(\mathrm{M}+\mathrm{H})^{+}$ 389.01453; found 389.01495 .

1-Benzyl-3-iodo-6-methoxy-1H-indole-2-carbonitrile (7c): White solid, 92\%, 2.40 g. ${ }^{1} \mathrm{H}$ $\operatorname{NMR}\left(300 \mathrm{MHz}, \mathrm{CDCl}_{3}\right): \delta=7.38-7.28(\mathrm{~m}, 4 \mathrm{H}, \mathrm{Ar}-\mathrm{H}), 7.17(\mathrm{dd}, J=7.6 \mathrm{~Hz}, J=2.1 \mathrm{~Hz}, 2 \mathrm{H}$, Ar- $H), 6.92(\mathrm{dd}, J=8.9 \mathrm{~Hz}, J=2.1 \mathrm{~Hz}, 1 \mathrm{H}, \mathrm{Ar}-H), 6.66(\mathrm{~d}, J=2.1 \mathrm{~Hz}, 1 \mathrm{H}, \mathrm{Ar}-H), 5.43(\mathrm{~s}, 2 \mathrm{H}$, $\left.\mathrm{CH}_{2}\right), 3.81\left(\mathrm{~s}, 3 \mathrm{H}, \mathrm{OCH}_{3}\right) \mathrm{ppm} .{ }^{13} \mathrm{C} \mathrm{NMR}\left(75 \mathrm{MHz}, \mathrm{CDCl}_{3}\right): \delta=160.4\left(\mathrm{C}_{\text {quat }}\right), 138.5$ (C $\left.\mathrm{C}_{\text {quat }}\right)$, $135.6\left(\mathrm{C}_{\text {quat }}\right), 129.2(\mathrm{Ar}-\mathrm{CH}), 128.4(\mathrm{Ar}-\mathrm{CH}), 127.0(\mathrm{Ar}-\mathrm{CH}), 124.2\left(\mathrm{C}_{\text {quat }}\right), 124.0(\mathrm{Ar}-\mathrm{CH})$, $114.2\left(\mathrm{C}_{\text {quat }}\right), 113.9(\mathrm{Ar}-\mathrm{CH}), 113.8\left(\mathrm{C}_{\text {quat }}\right), 92.9(\mathrm{Ar}-\mathrm{CH}), 70.9\left(\mathrm{C}_{\text {quat }}\right), 55.8\left(\mathrm{OCH}_{3}\right), 50.0$ $\left(\mathrm{CH}_{2}\right)$ ppm. HRMS (ESI): calcd. for $\mathrm{C}_{17} \mathrm{H}_{14} \mathrm{IN}_{2} \mathrm{O}(\mathrm{M}+\mathrm{H})^{+} 389.01508$; found 389.01492 .

1-Benzyl-5-fluoro-3-iodo-1H-indole-2-carbonitrile (7d): White solid, 76\%, 2.0 g. ${ }^{1} \mathrm{H}$ NMR $\left(300 \mathrm{MHz}, \mathrm{CDCl}_{3}\right): \delta=7.37-7.27(\mathrm{~m}, 4 \mathrm{H}, \mathrm{Ar}-H), 7.26-7.23(\mathrm{~m}, 1 \mathrm{H}, \mathrm{Ar}-\mathrm{H}), 7.19-7.11(\mathrm{~m}, 3 \mathrm{H}$, Ar- $H), 5.49\left(\mathrm{~s}, 2 \mathrm{H}, \mathrm{CH}_{2}\right) \mathrm{ppm} .{ }^{13} \mathrm{C} \mathrm{NMR}\left(75 \mathrm{MHz}, \mathrm{CDCl}_{3}\right): \delta=159.3\left(\mathrm{~d}, J=241.0 \mathrm{~Hz}, \mathrm{C}_{\text {quat }}\right)$, 135.3 ( $\left.\mathrm{C}_{\text {quat }}\right), 134.0\left(\mathrm{C}_{\text {quat }}\right), 130.3\left(\mathrm{~d}, J=10.6 \mathrm{~Hz}, \mathrm{C}_{\text {quat }}\right), 129.3(2 \mathrm{Ar}-\mathrm{CH}), 128.6(\mathrm{Ar}-\mathrm{CH})$, $126.9(2 \mathrm{Ar}-\mathrm{CH}), 116.9\left(\mathrm{C}_{\text {quat }}\right), 116.5(\mathrm{~d}, J=27.1 \mathrm{~Hz}, \mathrm{Ar}-\mathrm{CH}), 113.0\left(\mathrm{C}_{\text {quat }}\right), 112.4(\mathrm{~d}, J=$ $9.4 \mathrm{~Hz}, \mathrm{Ar}-\mathrm{CH}), 107.8(\mathrm{~d}, J=24.7 \mathrm{~Hz}, \mathrm{Ar}-\mathrm{CH}), 69.4\left(\mathrm{~d}, J=5.6 \mathrm{~Hz}, \mathrm{C}_{\text {quat }}\right), 50.4\left(\mathrm{CH}_{2}\right) \mathrm{ppm}$. ${ }^{19} \mathrm{~F}\left(282 \mathrm{MHz}, \mathrm{CDCl}_{3}\right): \delta=-119.9 \mathrm{ppm}$. HRMS (ESI): calcd. for $\mathrm{C}_{16} \mathrm{H}_{11} \mathrm{FIN}_{2}(\mathrm{M}+\mathrm{H})^{+}$ 376.99509; found 376.99497.

3.2.6. General Procedure for Sonogashira Coupling (Series 8)

A Schlenk tube was charged with phenylacetylene (1.08 mmol, 1.3 equiv.), $\mathrm{Et}_{3} \mathrm{~N}$ (0.34 mL, $2.52 \mathrm{mmol}, 3.0$ equiv.), DMF (3 mL), $\mathrm{PdCl}_{2}\left(\mathrm{PPh}_{3}\right)_{2}(58 \mathrm{mg}, 0.083 \mathrm{mmol}, 10 \mathrm{~mol} \%)$, 
compound 7a (300 mg, $0.837 \mathrm{mmol}, 1$ equiv.) and CuI (160 mg, $0.084 \mathrm{mmol}, 10 \mathrm{~mol} \%$ ). The tube was evacuated and backfilled with argon, and the mixture was stirred at room temperature overnight. Then, the mixture was hydrolyzed with sat. aq. $\mathrm{NH}_{4} \mathrm{Cl}(10 \mathrm{~mL})$, extracted by $\mathrm{Et}_{2} \mathrm{O}(3 \times 20 \mathrm{~mL})$, and the combined organic layers were washed with brine $(6 \times 10 \mathrm{~mL})$, dried over $\mathrm{MgSO}_{4}$ and concentrated under reduced pressure. The product was purified by column chromatography using petroleum ether/ethyl acetate (80:20) as eluent.

1-Benzyl-3-(phenylethynyl)-1H-indole-2-carbonitrile (8a): Yellow solid, 70\%, $195 \mathrm{mg} . \mathrm{mp}$ 125-127 ${ }^{\circ} \mathrm{C} .{ }^{1} \mathrm{H} \mathrm{NMR}\left(300 \mathrm{MHz}, \mathrm{CDCl}_{3}\right): \delta=7.88(\mathrm{dt}, J=8.0 \mathrm{~Hz}, J=1.0 \mathrm{~Hz}, 1 \mathrm{H}, \mathrm{Ar}-H)$, 7.67-7.62 (m, 2H, Ar-H), 7.44-7.39 (m, 4H, Ar-H), 7.36-7.28 (m, 5H, Ar-H), 7.20 (dd, J = $7.6 \mathrm{~Hz}, 1.9 \mathrm{~Hz}, 2 \mathrm{H}, \mathrm{Ar}-\mathrm{H}), 5.48\left(\mathrm{~s}, 2 \mathrm{H}, \mathrm{CH}_{2}\right) \mathrm{ppm} .{ }^{13} \mathrm{C} \mathrm{NMR}\left(75 \mathrm{MHz}^{\mathrm{C}} \mathrm{CDCl}_{3}\right): \delta=137.1$ (C quat $), 135.6$ ( $\left.\mathrm{C}_{\text {quat }}\right), 131.9$ (2Ar- $\left.\mathrm{CH}\right), 129.2(2 \mathrm{Ar}-\mathrm{CH}), 128.8(\mathrm{Ar}-\mathrm{CH}), 128.6(2 \mathrm{Ar}-\mathrm{CH}), 128.4$ (Ar-CH), 127.5 ( $\left.\mathrm{C}_{\text {quat }}\right), 127.0(\mathrm{Ar}-\mathrm{CH}), 127.0(2 \mathrm{Ar}-\mathrm{CH}), 123.0\left(\mathrm{C}_{\text {quat }}\right), 122.4(\mathrm{Ar}-\mathrm{CH}), 121.8$ (Ar-CH), 112.8 (2C quat), $111.1(\mathrm{Ar}-\mathrm{CH}), 109.8\left(\mathrm{C}_{\text {quat }}\right), 97.2\left(\mathrm{C}_{\text {quat }}\right), 79.6\left(\mathrm{C}_{\text {quat }}\right), 49.6\left(\mathrm{CH}_{2}\right)$ ppm. HRMS (ESI): calcd. for $\mathrm{C}_{24} \mathrm{H}_{17} \mathrm{~N}_{2}(\mathrm{M}+\mathrm{H})^{+} 333.13863$; found 333.13760.

1-Benzyl-3-(p-tolylethynyl)-1H-indole-2-carbonitrile (8b): Yellow solid, 81\%, $235 \mathrm{mg} . \mathrm{mp}$ 163-165 ${ }^{\circ} \mathrm{C} .{ }^{1} \mathrm{H}$ NMR $\left(300 \mathrm{MHz}, \mathrm{CDCl}_{3}\right): \delta=7.86(\mathrm{~d}, J=8.2 \mathrm{~Hz}, 1 \mathrm{H}, \mathrm{Ar}-H), 7.51(\mathrm{~d}, J=$ $8.1 \mathrm{~Hz}, 2 \mathrm{H}, \mathrm{Ar}-H), 7.43-7.26(\mathrm{~m}, 6 \mathrm{H}, \mathrm{Ar}-\mathrm{H}), 7.22-7.16(\mathrm{~m}, 3 \mathrm{H}, \mathrm{Ar}-\mathrm{H}), 5.48\left(\mathrm{~s}, 2 \mathrm{H}, \mathrm{CH}_{2}\right), 2.40$ (s, 3H, $\left.\mathrm{CH}_{3}\right)$ ppm. ${ }^{13} \mathrm{C} \mathrm{NMR}\left(75 \mathrm{MHz}, \mathrm{CDCl}_{3}\right): \delta=139.0\left(\mathrm{C}_{\text {quat }}\right), 137.1$ (C quat $), 135.6\left(\mathrm{C}_{\text {quat }}\right)$, 131.7 (Ar-CH), 129.3 (Ar-CH), 129.1 (Ar-CH), 128.4 (Ar-CH), 127.5 ( $\left.\mathrm{C}_{\text {quat }}\right), 127.0$ (Ar-CH), $122.3(\mathrm{Ar}-\mathrm{CH}), 121.8(\mathrm{Ar}-\mathrm{CH}), 119.9\left(\mathrm{C}_{\text {quat }}\right), 112.9$ ( $\left.\mathrm{C}_{\text {quat }}\right), 112.6\left(\mathrm{C}_{\text {quat }}\right), 111.0(\mathrm{Ar}-\mathrm{CH}), 110.1$ (C quat), 97.4 (C quat), $79.0\left(\mathrm{C}_{\text {quat }}\right), 49.5\left(\mathrm{CH}_{2}\right), 21.7\left(\mathrm{CH}_{3}\right)$ ppm. HRMS (ESI): calcd. for $\mathrm{C}_{25} \mathrm{H}_{19} \mathrm{~N}_{2}(\mathrm{M}+\mathrm{H})^{+} 347.15482$; found 346.15489.

1-Benzyl-3-((4-fluorophenyl)ethynyl)-1H-indole-2-carbonitrile (8c): Brown solid, 69\%, $202 \mathrm{mg} . \mathrm{mp} 180-182{ }^{\circ} \mathrm{C} .{ }^{1} \mathrm{H}$ NMR $\left(300 \mathrm{MHz} \mathrm{CDCl}_{3}\right): \delta=7.85(\mathrm{dt}, J=8.0 \mathrm{~Hz}, J=1.0 \mathrm{~Hz}$, $1 \mathrm{H}, \mathrm{Ar}-H), 7.60(\mathrm{dd}, J=8.9 \mathrm{~Hz}, J=5.4 \mathrm{~Hz}, 2 \mathrm{H}, \mathrm{Ar}-H), 7.40(\mathrm{ddd}, J=8.4 \mathrm{~Hz}, J=6.6 \mathrm{~Hz}, J=$ $1.2 \mathrm{~Hz}, 1 \mathrm{H}, \mathrm{Ar}-H), 7.35-7.27(\mathrm{~m}, 5 \mathrm{H}, \mathrm{Ar}-H), 7.21-7.18(\mathrm{~m}, 2 \mathrm{H}, \mathrm{Ar}-\mathrm{H}), 7.08(\mathrm{t}, J=8.7 \mathrm{~Hz}, 2 \mathrm{H}$, $\mathrm{Ar}-\mathrm{H}), 5.48\left(\mathrm{~s}, 2 \mathrm{H}, \mathrm{CH}_{2}\right)$ ppm. ${ }^{13} \mathrm{C} \mathrm{NMR}\left(75 \mathrm{MHz} \mathrm{CDCl}_{3}\right): \delta=162.9\left(\mathrm{~d}, J=250.3 \mathrm{~Hz}, \mathrm{C}_{\text {quat }}\right)$, 137.1 (C quat), 135.6 ( $\left.\mathrm{C}_{\text {quat }}\right), 133.8(\mathrm{~d}, J=8.4 \mathrm{~Hz}, \mathrm{Ar}-\mathrm{CH}), 129.2(2 \mathrm{Ar}-\mathrm{CH}), 128.5(\mathrm{Ar}-\mathrm{CH})$, 127.5 (C quat), 127.1 (Ar-CH), $127.0(2 \mathrm{Ar}-\mathrm{CH}), 122.4(\mathrm{Ar}-\mathrm{CH}), 121.7(\mathrm{Ar}-\mathrm{CH}), 119.1(\mathrm{~d}, J=$ $\left.3.5 \mathrm{~Hz}, \mathrm{C}_{\text {quat }}\right), 115.9$ (d, J = 22.1 Hz, $\left.\mathrm{Ar}-\mathrm{CH}\right), 112.9$ (C $\left.\mathrm{C}_{\text {quat }}\right), 112.8$ (C $\left.\mathrm{C}_{\text {quat }}\right), 111.1(\mathrm{Ar}-\mathrm{CH}), 109.7$ (C quat), 96.0 ( $\left.\mathrm{C}_{\text {quat }}\right), 79.4\left(\mathrm{C}_{\text {quat }}\right), 49.6\left(\mathrm{CH}_{2}\right) \mathrm{ppm} .{ }^{19} \mathrm{~F} \mathrm{NMR}\left(282 \mathrm{MHz}, \mathrm{CDCl}_{3}\right): \delta=-110.2$ ppm. HRMS (ESI): calcd. for $\mathrm{C}_{24} \mathrm{H}_{16} \mathrm{FN}_{2}(\mathrm{M}-\mathrm{H})^{+}$351.12920; found 351.12816.

1-Benzyl-3-((4-methoxyphenyl)ethynyl)-1H-indole-2-carbonitrile (8d): Brown solid, 83\%, $252 \mathrm{mg}$. mp 137-139 ${ }^{\circ} \mathrm{C} .{ }^{1} \mathrm{H}$ NMR $\left(300 \mathrm{MHz}, \mathrm{CDCl}_{3}\right): \delta=7.85(\mathrm{dt}, J=8.0 \mathrm{~Hz}, J=1.0 \mathrm{~Hz}, 1 \mathrm{H}$, Ar-H), $7.56(\mathrm{~d}, J=8.9 \mathrm{~Hz}, 2 \mathrm{H}, \mathrm{Ar}-H), 7.40(\mathrm{ddd}, J=8.4 \mathrm{~Hz}, J=6.7 \mathrm{~Hz}, J=1.2 \mathrm{~Hz}, 1 \mathrm{H}, \mathrm{Ar}-H)$, 7.36-7.24 (m, 6H, Ar-H), $7.19(\mathrm{dd}, J=7.8 \mathrm{~Hz}, J=2.0 \mathrm{~Hz}, 1 \mathrm{H}, \mathrm{Ar}-\mathrm{H}), 6.91(\mathrm{~d}, J=8.9 \mathrm{~Hz}, 2 \mathrm{H}$, $\mathrm{Ar}-\mathrm{H}), 5.47\left(\mathrm{~s}, 2 \mathrm{H}, \mathrm{CH}_{2}\right), 3.85\left(\mathrm{~s}, 3 \mathrm{H}, \mathrm{OCH}_{3}\right) \mathrm{ppm} .{ }^{13} \mathrm{C} \mathrm{NMR}\left(75 \mathrm{MHz}, \mathrm{CDCl}_{3}\right): \delta=160.0$ (C $\left.\mathrm{C}_{\text {quat }}\right), 137.0$ ( $\left.\mathrm{C}_{\text {quat }}\right), 135.6$ ( $\left.\mathrm{C}_{\text {quat }}\right), 133.3$ (2Ar-CH), $129.1(2 \mathrm{Ar}-\mathrm{CH}), 128.3(\mathrm{Ar}-\mathrm{CH}), 127.4$ (C quat), 126.9 (2Ar-CH), $122.2(\mathrm{Ar}-\mathrm{CH}), 121.7$ (Ar-CH), 115.0 (C quat $), 114.2(\mathrm{Ar}-\mathrm{CH}), 112.9$ ( $\left.\mathrm{C}_{\text {quat }}\right), 112.4$ ( $\left.\mathrm{C}_{\text {quat }}\right), 111.0(\mathrm{Ar}-\mathrm{CH}), 110.2$ ( $\left.\mathrm{C}_{\text {quat }}\right), 97.2$ ( $\left.\mathrm{C}_{\text {quat }}\right), 78.3\left(\mathrm{C}_{\text {quat }}\right), 55.4\left(\mathrm{OCH}_{3}\right)$, $49.4\left(\mathrm{CH}_{2}\right)$ ppm. HRMS (ESI): calcd. for $\mathrm{C}_{25} \mathrm{H}_{19} \mathrm{~N}_{2} \mathrm{O}(\mathrm{M}+\mathrm{H})^{+} 363.14919$; found 363.14962.

1-Benzyl-3-((4-ethylphenyl)ethynyl)-1H-indole-2-carbonitrile (8e): Brown solid, 83\%, 251 mg. mp 94-96 ${ }^{\circ} \mathrm{C} .{ }^{1} \mathrm{H} \mathrm{NMR}\left(300 \mathrm{MHz}, \mathrm{CDCl}_{3}\right): \delta=7.86(\mathrm{dt}, J=8.0 \mathrm{~Hz}, J=1.0 \mathrm{~Hz}, 1 \mathrm{H}$, Ar- $H), 7.54(\mathrm{~d}, J=8.3 \mathrm{~Hz}, 2 \mathrm{H}, \mathrm{Ar}-H), 7.40(\mathrm{ddd}, J=8.4 \mathrm{~Hz}, J=6.6 \mathrm{~Hz}, J=1.2 \mathrm{~Hz}, 1 \mathrm{H}, \mathrm{Ar}-H)$, 7.36-7.28 (m, 5H, Ar-H), 7.24-7.18 (m, 4H, Ar-H), $5.48\left(\mathrm{~s}, 2 \mathrm{H}, \mathrm{CH}_{2}\right), 2.69$ (q, J = 7.6 Hz, 2H, $\left.\mathrm{CH}_{2}\right), 1.26\left(\mathrm{t}, J=7.6 \mathrm{~Hz}, 3 \mathrm{H}, \mathrm{CH}_{3}\right) \mathrm{ppm} .{ }^{13} \mathrm{C} \mathrm{NMR}\left(75 \mathrm{MHz}_{2} \mathrm{CDCl}_{3}\right): \delta=145.3\left(\mathrm{C}_{\text {quat }}\right), 137.0$ (C quat), 135.6 ( $\left.\mathrm{C}_{\text {quat }}\right), 131.8(2 \mathrm{Ar}-\mathrm{CH}), 129.1(2 \mathrm{Ar}-\mathrm{CH}), 128.4(\mathrm{Ar}-\mathrm{CH}), 128.1(2 \mathrm{Ar}-\mathrm{CH}), 127.5$ (C $\left.\mathrm{C}_{\text {quat }}\right), 127.0(3 \mathrm{Ar}-\mathrm{CH}), 122.3(\mathrm{Ar}-\mathrm{CH}), 121.8(\mathrm{Ar}-\mathrm{CH}), 120.1$ ( $\left.\mathrm{C}_{\text {quat }}\right), 112.9\left(\mathrm{C}_{\text {quat }}\right), 112.6$ (C $\left.\mathrm{C}_{\text {quat }}\right), 111.0(\mathrm{Ar}-\mathrm{CH}), 110.1$ ( $\left.\mathrm{C}_{\text {quat }}\right), 97.4\left(\mathrm{C}_{\text {quat }}\right), 79.0\left(\mathrm{C}_{\text {quat }}\right), 49.5\left(\mathrm{CH}_{2}\right), 29.0\left(\mathrm{CH}_{2}\right), 15.5$ $\left(\mathrm{CH}_{3}\right)$ ppm. HRMS (ESI): calcd. for $\mathrm{C}_{26} \mathrm{H}_{21} \mathrm{~N}_{2}(\mathrm{M}+\mathrm{H})^{+}$361.16993; found 361.16903.

1-Benzyl-3-(pyridin-3-yl-ethynyl)-1H-indole-2-carbonitrile (8f): Brown solid, 78\%, $216 \mathrm{mg}$. mp 138-140 ${ }^{\circ} \mathrm{C} .{ }^{1} \mathrm{H}$ NMR $\left(300 \mathrm{MHz}, \mathrm{CDCl}_{3}\right): \delta=8.67(\mathrm{ddd}, J=4.9 \mathrm{~Hz}, J=1.8 \mathrm{~Hz}, J=1.0 \mathrm{~Hz}$, $1 \mathrm{H}, \mathrm{Ar}-H), 7.93(\mathrm{dt}, J=8.1 \mathrm{~Hz}, J=1.0 \mathrm{~Hz}, 1 \mathrm{H}, \mathrm{Ar}-H), 7.72(\mathrm{dt}, J=7.8 \mathrm{~Hz}, J=1.8 \mathrm{~Hz}, 1 \mathrm{H}$, 
Ar- $H), 7.63(\mathrm{dt}, J=7.8 \mathrm{~Hz}, J=1.2 \mathrm{~Hz}, 1 \mathrm{H}, \mathrm{Ar}-H), 7.41(\mathrm{ddd}, J=8.4 \mathrm{~Hz}, J=6.6 \mathrm{~Hz}, J=1.2 \mathrm{~Hz}$, $1 \mathrm{H}, \mathrm{Ar}-\mathrm{H}), 7.37-7.26(\mathrm{~m}, 6 \mathrm{H}, \mathrm{Ar}-\mathrm{H}), 7.19(\mathrm{dd}, J=7.7 \mathrm{~Hz}, J=2.1 \mathrm{~Hz}, 2 \mathrm{H}, \mathrm{Ar}-\mathrm{H}), 5.50(\mathrm{~s}, 2 \mathrm{H}$, $\left.\mathrm{CH}_{2}\right) \mathrm{ppm} .{ }^{13} \mathrm{C} \mathrm{NMR}\left(75 \mathrm{MHz}, \mathrm{CDCl}_{3}\right): \delta=150.1(\mathrm{Ar}-\mathrm{CH}), 143.0\left(\mathrm{C}_{\text {quat }}\right), 136.9\left(\mathrm{C}_{\text {quat }}\right), 136.2$ (Ar-CH), $135.3\left(\mathrm{C}_{\text {quat }}\right), 129.1(2 \mathrm{Ar}-\mathrm{CH}), 128.3(\mathrm{Ar}-\mathrm{CH}), 127.6(\mathrm{Ar}-\mathrm{CH}), 127.4\left(\mathrm{C}_{\text {quat }}\right), 127.0$ (Ar-CH), $126.8(2 \mathrm{Ar}-\mathrm{CH}), 123.0(\mathrm{Ar}-\mathrm{CH}), 122.5(\mathrm{Ar}-\mathrm{CH}), 121.8(\mathrm{Ar}-\mathrm{CH}), 113.5\left(\mathrm{C}_{\text {quat }}\right), 112.4$ $\left(\mathrm{C}_{\text {quat }}\right), 111.0(\mathrm{Ar}-\mathrm{CH}), 108.5\left(\mathrm{C}_{\text {quat }}\right), 95.9\left(\mathrm{C}_{\text {quat }}\right), 79.5\left(\mathrm{C}_{\text {quat }}\right), 49.5\left(\mathrm{CH}_{2}\right) \mathrm{ppm}$. HRMS (ESI): calcd. for $\mathrm{C}_{23} \mathrm{H}_{16} \mathrm{~N}_{3}(\mathrm{M}+\mathrm{H})^{+}$334.13387; found 334.13301.

1-Benzyl-3-((2-fluorophenyl)ethynyl)-1H-indole-2-carbonitrile (8g): Brown solid, 76\%, 222 mg. mp 178-180 ${ }^{\circ} \mathrm{C} .{ }^{1} \mathrm{H}$ NMR $\left(300 \mathrm{MHz}, \mathrm{CDCl}_{3}\right): \delta=7.88(\mathrm{dt}, J=8.0 \mathrm{~Hz}, J=0.9 \mathrm{~Hz}, 1 \mathrm{H}$, Ar- $H), 7.60(\mathrm{td}, J=7.2 \mathrm{~Hz}, J=1.9 \mathrm{~Hz}, 1 \mathrm{H}, \mathrm{Ar}-H), 7.41(\mathrm{ddd}, J=8.4 \mathrm{~Hz}, J=6.7 \mathrm{~Hz}, J=$ $1.2 \mathrm{~Hz}, 1 \mathrm{H}, \mathrm{Ar}-\mathrm{H}), 7.38-7.27(\mathrm{~m}, 6 \mathrm{H}, \mathrm{Ar}-\mathrm{H}), 7.21-7.11(\mathrm{~m}, 4 \mathrm{H}, \mathrm{Ar}-\mathrm{H}), 5.50\left(\mathrm{~s}, 2 \mathrm{H}, \mathrm{CH}_{2}\right)$ ppm. ${ }^{13} \mathrm{C} \mathrm{NMR}\left(75 \mathrm{MHz}, \mathrm{CDCl}_{3}\right): \delta=162.7\left(\mathrm{~d}, J=252.3 \mathrm{~Hz}, \mathrm{C}_{\text {quat }}\right), 137.0$ (C $\left.\mathrm{C}_{\text {quat }}\right), 135.5$ ( $\left.\mathrm{C}_{\text {quat }}\right), 133.5(\mathrm{Ar}-\mathrm{CH}), 130.5(\mathrm{~d}, J=7.9 \mathrm{~Hz}, \mathrm{Ar}-\mathrm{CH}), 129.1(2 \mathrm{Ar}-\mathrm{CH}), 128.4(\mathrm{Ar}-\mathrm{CH}), 127.5$ ( $\left.\mathrm{C}_{\text {quat }}\right), 127.1(\mathrm{Ar}-\mathrm{CH}), 127.0(2 \mathrm{Ar}-\mathrm{CH}), 124.1(\mathrm{~d}, J=3.7 \mathrm{~Hz}, \mathrm{Ar}-\mathrm{CH}), 122.6(\mathrm{Ar}-\mathrm{CH}), 121.8$ (Ar-CH), $115.7(\mathrm{~d}, J=20.7 \mathrm{~Hz}, \mathrm{Ar}-\mathrm{CH}), 112.8\left(\mathrm{C}_{\text {quat }}\right), 112.7\left(\mathrm{C}_{\text {quat }}\right), 111.6(\mathrm{~d}, J=15.7 \mathrm{~Hz}$, $\left.\mathrm{C}_{\text {quat }}\right), 111.1(\mathrm{Ar}-\mathrm{CH}), 109.3\left(\mathrm{C}_{\text {quat }}\right), 90.4\left(\mathrm{C}_{\text {quat }}\right), 84.7\left(\mathrm{C}_{\text {quat }}\right), 49.5\left(\mathrm{CH}_{2}\right) \mathrm{ppm} .{ }^{19} \mathrm{~F} \mathrm{NMR}(282$ $\left.\mathrm{MHz}, \mathrm{CDCl}_{3}\right): \delta=-112.1 \mathrm{ppm}$. HRMS (ESI): calcd. for $\mathrm{C}_{24} \mathrm{H}_{16} \mathrm{FN}_{2}(\mathrm{M}+\mathrm{H})^{+} 351.12920$; found 351.12956 .

1-Benzyl-5-methoxy-3-((4-methoxyphenyl)ethynyl)-1H-indole-2-carbonitrile (8h): Brown solid, 90\%, 272 mg. ${ }^{1} \mathrm{H}$ NMR ( $300 \mathrm{MHz}, \mathrm{CDCl}_{3}$ ): $\delta=7.57$ (d, J = $\left.8.9 \mathrm{~Hz}, 2 \mathrm{H}, \mathrm{Ar}-\mathrm{H}\right), 7.35-7.28$ $(\mathrm{m}, 3 \mathrm{H}, \mathrm{Ar}-\mathrm{H}), 7.20(\mathrm{~d}, J=9.1 \mathrm{~Hz}, 1 \mathrm{H}, \mathrm{Ar}-H), 7.16(\mathrm{dd}, J=7.4 \mathrm{~Hz}, J=2.4 \mathrm{~Hz}, 2 \mathrm{H}, \mathrm{Ar}-\mathrm{H}), 7.04$ $(\mathrm{dd}, J=9.1 \mathrm{~Hz}, J 2.5 \mathrm{~Hz}, 1 \mathrm{H}, \mathrm{Ar}-H), 6.91(\mathrm{~d}, J=8.9 \mathrm{~Hz}, 2 \mathrm{H}, \mathrm{Ar}-\mathrm{H}), 5.44\left(\mathrm{~s}, 2 \mathrm{H}, \mathrm{CH}_{2}\right), 3.89(\mathrm{~s}$, $\left.3 \mathrm{H}, \mathrm{OCH}_{3}\right), 3.85\left(\mathrm{~s}, 3 \mathrm{H}, \mathrm{OCH}_{3}\right) \mathrm{ppm} .{ }^{13} \mathrm{C} \mathrm{NMR}\left(75 \mathrm{MHz}, \mathrm{CDCl}_{3}\right): \delta=160.0\left(\mathrm{C}_{\text {quat }}\right), 156.1$ ( $\left.\mathrm{C}_{\text {quat }}\right), 135.7\left(\mathrm{C}_{\text {quat }}\right), 133.4(2 \mathrm{Ar}-\mathrm{CH}), 132.4\left(\mathrm{C}_{\text {quat }}\right), 129.1(2 \mathrm{Ar}-\mathrm{CH}), 128.4(\mathrm{Ar}-\mathrm{CH}), 128.1$ $\left(\mathrm{C}_{\text {quat }}\right), 126.9(2 \mathrm{Ar}-\mathrm{CH}), 118.6(\mathrm{Ar}-\mathrm{CH}), 115.1\left(\mathrm{C}_{\text {quat }}\right), 114.2(2 \mathrm{Ar}-\mathrm{CH}), 113.0\left(\mathrm{C}_{\text {quat }}\right), 112.6$ $\left(\mathrm{C}_{\text {quat }}\right), 112.1(\mathrm{Ar}-\mathrm{CH}), 109.3\left(\mathrm{C}_{\text {quat }}\right), 101.6(\mathrm{Ar}-\mathrm{CH}), 97.0\left(\mathrm{C}_{\text {quat }}\right), 78.4\left(\mathrm{C}_{\text {quat }}\right), 55.9\left(\mathrm{OCH}_{3}\right)$, $55.5\left(\mathrm{OCH}_{3}\right), 49.7\left(\mathrm{CH}_{2}\right)$ ppm. HRMS (ESI): calcd. for $\mathrm{C}_{26} \mathrm{H}_{21} \mathrm{~N}_{2} \mathrm{O}_{2}(\mathrm{M}+\mathrm{H})^{+} 393.15975$; found 393.16029 .

1-Benzyl-6-methoxy-3-((2-methoxyphenyl)ethynyl)-1H-indole-2-carbonitrile (8i): Brown solid, 83\%, $252 \mathrm{mg} .{ }^{1} \mathrm{H} \mathrm{NMR}\left(300 \mathrm{MHz}, \mathrm{CDCl}_{3}\right): \delta=7.74(\mathrm{~d}, J=8.8 \mathrm{~Hz}, 1 \mathrm{H}, \mathrm{Ar}-H), 7.56(\mathrm{dd}$, $J=7.6 \mathrm{~Hz}, J=1.6 \mathrm{~Hz}, 1 \mathrm{H}, \mathrm{Ar}-H), 7.39-7.28(\mathrm{~m}, 4 \mathrm{H}, \mathrm{Ar}-H), 7.17(\mathrm{dd}, J=7.6 \mathrm{~Hz}, J=1.6 \mathrm{~Hz}$, 2H, Ar-H), $6.98(\mathrm{dd}, J=7.6 \mathrm{~Hz}, \mathrm{~J}=1.0 \mathrm{~Hz}, 1 \mathrm{H}, \mathrm{Ar}-H), 6.96-6.90(\mathrm{~m}, 2 \mathrm{H}, \mathrm{Ar}-H), 6.67(\mathrm{~d}, J=$ $2.1 \mathrm{~Hz}, 1 \mathrm{H}, \mathrm{Ar}-\mathrm{H}), 5.41\left(\mathrm{~s}, 2 \mathrm{H}, \mathrm{CH}_{2}\right), 3.96\left(\mathrm{~s}, 3 \mathrm{H}, \mathrm{OCH}_{3}\right), 3.80\left(\mathrm{~s}, 3 \mathrm{H}, \mathrm{OCH}_{3}\right) \mathrm{ppm} .{ }^{13} \mathrm{C} \mathrm{NMR}$ $\left(75 \mathrm{MHz}, \mathrm{CDCl}_{3}\right): \delta=160.3\left(\mathrm{C}_{\text {quat }}\right), 160.1$ ( $\left.\mathrm{C}_{\text {quat }}\right), 138.2\left(\mathrm{C}_{\text {quat }}\right), 135.7\left(\mathrm{C}_{\text {quat }}\right), 133.5(\mathrm{Ar}-\mathrm{CH})$, 130.2 (Ar-CH), $129.1(2 \mathrm{Ar}-\mathrm{CH}), 128.3(\mathrm{Ar}-\mathrm{CH}), 126.9(2 \mathrm{Ar}-\mathrm{CH}), 122.7(\mathrm{Ar}-\mathrm{CH}), 121.8\left(\mathrm{C}_{\text {quat }}\right)$, 120.6 (Ar-CH), $113.4(\mathrm{Ar}-\mathrm{CH}), 113.2\left(\mathrm{C}_{\text {quat }}\right), 112.4\left(\mathrm{C}_{\text {quat }}\right), 111.4\left(\mathrm{C}_{\text {quat }}\right), 111.0(\mathrm{Ar}-\mathrm{CH}), 110.5$ $\left(\mathrm{C}_{\text {quat }}\right)$, $93.5\left(\mathrm{C}_{\text {quat }}\right)$, 93.2 (Ar- $\left.\mathrm{CH}\right), 83.8\left(\mathrm{C}_{\text {quat }}\right), 56.0\left(\mathrm{OCH}_{3}\right), 55.7\left(\mathrm{OCH}_{3}\right), 49.4\left(\mathrm{CH}_{2}\right) \mathrm{ppm}$. HRMS (ESI): calcd. for $\mathrm{C}_{26} \mathrm{H}_{21} \mathrm{~N}_{2} \mathrm{O}_{2}(\mathrm{M}+\mathrm{H})^{+}$393.15975; found 393.15951.

1-Benzyl-5-fluoro-3-(m-tolylethynyl)-1H-indole-2-carbonitrile (8j): Brown solid, 86\%, 250 mg. ${ }^{1} \mathrm{H}$ NMR $\left(300 \mathrm{MHz}, \mathrm{CDCl}_{3}\right): \delta=7.55-7.46(\mathrm{~m}, 3 \mathrm{H}), 7.38-7.27(\mathrm{~m}, 4 \mathrm{H}), 7.25-7.08(\mathrm{~m}, 5 \mathrm{H})$, $5.46(\mathrm{~s}, 2 \mathrm{H}), 2.40(\mathrm{~s}, 3 \mathrm{H}) \mathrm{ppm} .{ }^{13} \mathrm{C} \mathrm{NMR}\left(75 \mathrm{MHz}, \mathrm{CDCl}_{3}\right): \delta=159.2\left(\mathrm{~d}, J=241.2 \mathrm{~Hz}, \mathrm{C}_{\text {quat }}\right)$, $139.2\left(\mathrm{C}_{\text {quat }}\right), 135.4\left(\mathrm{C}_{\text {quat }}\right), 133.7\left(\mathrm{C}_{\text {quat }}\right), 131.8(2 \mathrm{Ar}-\mathrm{CH}), 129.4(2 \mathrm{Ar}-\mathrm{CH}), 129.3(2 \mathrm{Ar}-\mathrm{CH})$, $128.6(\mathrm{Ar}-\mathrm{CH}), 128.1\left(\mathrm{~d}, J=10.4 \mathrm{~Hz}, \mathrm{C}_{\text {quat }}\right), 126.9(2 \mathrm{Ar}-\mathrm{CH}), 119.7\left(\mathrm{C}_{\text {quat }}\right), 116.2(\mathrm{~d}, J=$ $27.0 \mathrm{~Hz}, \mathrm{Ar}-\mathrm{CH}), 114.0\left(\mathrm{C}_{\text {quat }}\right), 112.5\left(\mathrm{C}_{\text {quat }}\right), 112.3(\mathrm{~d}, J=9.1 \mathrm{~Hz}, \mathrm{Ar}-\mathrm{CH}), 109.8(\mathrm{~d}, J=5.4 \mathrm{~Hz}$, $\left.\mathrm{C}_{\text {quat }}\right), 106.5(\mathrm{~d}, \mathrm{~J}=24.0 \mathrm{~Hz}), 97.8\left(\mathrm{C}_{\text {quat }}\right), 78.4\left(\mathrm{C}_{\text {quat }}\right), 49.9\left(\mathrm{CH}_{2}\right), 21.7\left(\mathrm{CH}_{3}\right) \mathrm{ppm} .{ }^{19} \mathrm{~F} \mathrm{NMR}$ $\left(282 \mathrm{MHz}, \mathrm{CDCl}_{3}\right): \delta=-120.3 \mathrm{ppm}$. HRMS (ESI): calcd. for $\mathrm{C}_{25} \mathrm{H}_{18} \mathrm{FN}_{2}(\mathrm{M}+\mathrm{H})^{+} 365.14540$; found 365.14514 .

\subsubsection{General Procedure for Suzuki Coupling (Series 9)}

A Schlenk tube was charged with toluene $(3 \mathrm{~mL})$, ethanol $(2 \mathrm{~mL})$, sat. aq. $\mathrm{NaHCO}_{3}$ (1.5 mL), compound $7 \mathbf{a}(300 \mathrm{mg}, 0.83 \mathrm{mmol}, 1$ equiv.) and boronic acid (1.2 mmol, 1.5 equiv.). The tube was vigorously stirred for few minutes and $\mathrm{Pd}\left(\mathrm{PPh}_{3}\right)_{4}(90 \mathrm{mg}, 0.083$ $\mathrm{mmol}, 10 \mathrm{~mol} \%$ ) was added to the solution. The mixture was heated at $130^{\circ} \mathrm{C}$ for $4 \mathrm{~h}$ and then cooled to room temperature. The mixture was extracted with EtOAc $(3 \times 10 \mathrm{~mL})$, and 
the combined organic layers were washed with water $(2 \times 10 \mathrm{~mL})$ and dried over $\mathrm{MgSO}_{4}$. Solvents were removed under reduced pressure, and the residue was purified by column chromatography using petroleum ether/EtOAc as eluent (80:20).

1-Benzyl-3-(4-methoxyphenyl)-1H-indole-2-carbonitrile (9a): Brown solid, 79\%, $221 \mathrm{mg}$. mp 153-155 ${ }^{\circ} \mathrm{C} .{ }^{1} \mathrm{H}$ NMR $\left(300 \mathrm{MHz}, \mathrm{CDCl}_{3}\right): \delta=7.85(\mathrm{dt}, J=8.2 \mathrm{~Hz}, J=0.9 \mathrm{~Hz}, 1 \mathrm{H}, \mathrm{Ar}-\mathrm{H})$, 7.67 (d, J = 8.9 Hz, 2H, Ar-H), 7.39-7.35 (m, 2H, Ar-H), 7.33-7.28 (m, 3H, Ar-H), 7.26-7.20 $(\mathrm{m}, 3 \mathrm{H}, \mathrm{Ar}-\mathrm{H}), 7.07(\mathrm{~d}, J=8.9 \mathrm{~Hz}, 2 \mathrm{H}, \mathrm{Ar}-\mathrm{H}), 5.52\left(\mathrm{~s}, 2 \mathrm{H}, \mathrm{CH}_{2}\right), 3.89\left(\mathrm{~s}, 3 \mathrm{H}, \mathrm{OCH}_{3}\right) \mathrm{ppm}$. ${ }^{13} \mathrm{C}$ NMR $\left(75 \mathrm{MHz}, \mathrm{CDCl}_{3}\right): \delta=159.7\left(\mathrm{C}_{\text {quat }}\right), 138.0$ ( $\left.\mathrm{C}_{\text {quat }}\right), 136.2\left(\mathrm{C}_{\text {quat }}\right), 130.1(2 \mathrm{Ar}-\mathrm{CH})$, $129.1(2 \mathrm{Ar}-\mathrm{CH}), 128.5\left(\mathrm{C}_{\text {quat }}\right), 128.2(2 \mathrm{Ar}-\mathrm{CH}), 127.1(2 \mathrm{Ar}-\mathrm{CH}), 126.5(\mathrm{Ar}-\mathrm{CH}), 125.3\left(\mathrm{C}_{\text {quat }}\right)$, 124.4 ( $\left.\mathrm{C}_{\text {quat }}\right), 121.8(2 \mathrm{Ar}-\mathrm{CH}), 114.7(2 \mathrm{Ar}-\mathrm{CH}), 114.5\left(\mathrm{C}_{\text {quat }}\right), 110.9(\mathrm{Ar}-\mathrm{CH}), 107.2\left(\mathrm{C}_{\text {quat }}\right)$, $55.5\left(\mathrm{OCH}_{3}\right), 49.2\left(\mathrm{CH}_{2}\right)$ ppm. HRMS (ESI): calcd. for $\mathrm{C}_{23} \mathrm{H}_{19} \mathrm{~N}_{2} \mathrm{O}(\mathrm{M}+\mathrm{H})^{+} 339.14919$; found 339.14965 .

1-Benzyl-3-(4-fluorophenyl)-1H-indole-2-carbonitrile (9b): Brown solid, $81 \%, 220 \mathrm{mg}$. $\mathrm{mp}$ $120-122{ }^{\circ} \mathrm{C} .{ }^{1} \mathrm{H}$ NMR $\left(300 \mathrm{MHz}, \mathrm{CDCl}_{3}\right): \delta=7.82(\mathrm{dt}, J=8.2 \mathrm{~Hz}, J=0.9 \mathrm{~Hz}, 1 \mathrm{H}, \mathrm{Ar}-H), 7.70$ $(\mathrm{dd}, J=8.8 \mathrm{~Hz}, J=5.3 \mathrm{~Hz}, 2 \mathrm{H}, \mathrm{Ar}-H), 7.42-7.39(\mathrm{~m}, 2 \mathrm{H}, \mathrm{Ar}-H), 7.37-7.27(\mathrm{~m}, 3 \mathrm{H}, \mathrm{Ar}-H)$, 7.25-7.19 (m, 3H, Ar-H), $5.53\left(\mathrm{~s}, 2 \mathrm{H}, \mathrm{CH}_{2}\right) \mathrm{ppm} .{ }^{13} \mathrm{C} \mathrm{NMR}\left(75 \mathrm{MHz}, \mathrm{CDCl}_{3}\right): \delta=162.6(\mathrm{~d}, J$ $\left.=248.0 \mathrm{~Hz}, \mathrm{C}_{\text {quat }}\right), 137.9\left(\mathrm{C}_{\text {quat }}\right), 136.0\left(\mathrm{C}_{\text {quat }}\right), 130.6(\mathrm{~d}, \mathrm{~J}=8.1 \mathrm{~Hz}, \mathrm{Ar}-\mathrm{CH}), 129.1(2 \mathrm{Ar}-\mathrm{CH})$, $128.3(\mathrm{Ar}-\mathrm{CH}), 127.9\left(\mathrm{~d}, J=3.3 \mathrm{~Hz}, \mathrm{C}_{\text {quat }}\right), 127.5\left(\mathrm{C}_{\text {quat }}\right), 127.0(2 \mathrm{Ar}-\mathrm{CH}), 126.6(\mathrm{Ar}-\mathrm{CH})$, $125.1\left(\mathrm{C}_{\text {quat }}\right), 122.1(\mathrm{Ar}-\mathrm{CH}), 121.4(\mathrm{Ar}-\mathrm{CH}), 116.2(\mathrm{~d}, J=21.6 \mathrm{~Hz}, \mathrm{Ar}-\mathrm{CH}), 114.1$ ( $\left.\mathrm{C}_{\text {quat }}\right)$, 111.0 (Ar-CH), $107.5\left(\mathrm{C}_{\text {quat }}\right), 49.2\left(\mathrm{CH}_{2}\right) \mathrm{ppm} .{ }^{19} \mathrm{~F} \mathrm{NMR}\left(282 \mathrm{MHz}, \mathrm{CDCl}_{3}\right): \delta=-113.2 \mathrm{ppm}$. HRMS (ESI): calcd. for $\mathrm{C}_{22} \mathrm{H}_{16} \mathrm{FN}_{2}(\mathrm{M}+\mathrm{H})^{+} 327.12975$; found 327.12988.

1-Benzyl-3-(4-(tert-butyl)phenyl)-1H-indole-2-carbonitrile (9c): Brown solid, 83\%, $252 \mathrm{mg}$. mp 149-151 ${ }^{\circ} \mathrm{C} .{ }^{1} \mathrm{H}$ NMR $\left(300 \mathrm{MHz}, \mathrm{CDCl}_{3}\right): \delta=7.92(\mathrm{dt}, J=8.2 \mathrm{~Hz}, J=0.8 \mathrm{~Hz}, 1 \mathrm{H}, \mathrm{Ar}-H)$, $7.72(\mathrm{~d}, J=8.5 \mathrm{~Hz}, 2 \mathrm{H}, \mathrm{Ar}-H), 7.57(\mathrm{~d}, J=8.5 \mathrm{~Hz}, 2 \mathrm{H}, \mathrm{Ar}-H), 7.40-7.37(\mathrm{~m}, 2 \mathrm{H}, \mathrm{Ar}-H)$, 7.35-7.207 (m, 6H, Ar-H), $5.51\left(\mathrm{~s}, 2 \mathrm{H}, \mathrm{CH}_{2}\right), 1.41\left(\mathrm{~s}, 9 \mathrm{H}, \mathrm{C}\left(\mathrm{CH}_{3}\right)_{3}\right) \mathrm{ppm} .{ }^{13} \mathrm{C} \mathrm{NMR}(75 \mathrm{MHz}$, $\left.\mathrm{CDCl}_{3}\right): \delta=151.2\left(\mathrm{C}_{\text {quat }}\right), 138.0\left(\mathrm{C}_{\text {quat }}\right), 136.2\left(\mathrm{C}_{\text {quat }}\right), 129.1(2 \mathrm{Ar}-\mathrm{CH}), 129.0\left(\mathrm{C}_{\text {quat }}\right), 128.6$ ( $\left.\mathrm{C}_{\text {quat }}\right), 128.5(2 \mathrm{Ar}-\mathrm{CH}), 128.2(\mathrm{Ar}-\mathrm{CH}), 127.0(2 \mathrm{Ar}-\mathrm{CH}), 126.5(\mathrm{Ar}-\mathrm{CH}), 126.1(2 \mathrm{Ar}-\mathrm{CH}), 125.0$ $\left(\mathrm{C}_{\text {quat }}\right), 121.9(\mathrm{Ar}-\mathrm{CH}), 121.8(\mathrm{Ar}-\mathrm{CH}), 114.5\left(\mathrm{C}_{\text {quat }}\right), 111.0(\mathrm{Ar}-\mathrm{CH}), 107.4\left(\mathrm{C}_{\text {quat }}\right), 49.1\left(\mathrm{CH}_{2}\right)$, $34.8\left(\mathrm{C}_{\text {quat }}\right)$, $31.4\left(\mathrm{C}\left(\mathrm{CH}_{3}\right)_{3}\right)$ ppm. HRMS (ESI): calcd. for $\mathrm{C}_{26} \mathrm{H}_{25} \mathrm{~N}_{2}(\mathrm{M}+\mathrm{H})^{+}$365.20177; found 365.20152 .

1-Benzyl-3-(4-(methylthio)phenyl)-1H-indole-2-carbonitrile (9d): Yellow viscous liquid, $89 \%, 263 \mathrm{mg} .{ }^{1} \mathrm{H}$ NMR $\left(300 \mathrm{MHz}, \mathrm{CDCl}_{3}\right): \delta=7.85(\mathrm{dd}, J=8.2 \mathrm{~Hz}, J=0.9 \mathrm{~Hz}, 1 \mathrm{H}, \mathrm{Ar}-H)$, $7.67(\mathrm{~d}, J=8.6 \mathrm{~Hz}, 2 \mathrm{H}, \mathrm{Ar}-H), 7.42-7.38(\mathrm{~m}, 4 \mathrm{H}, \mathrm{Ar}-H), 7.36-7.29(\mathrm{~m}, 3 \mathrm{H}, \mathrm{Ar}-H), 7.26-7.22$ $(\mathrm{m}, 3 \mathrm{H}, \mathrm{Ar}-\mathrm{H}), 5.53\left(\mathrm{~s}, 2 \mathrm{H}, \mathrm{CH}_{2}\right), 2.55\left(\mathrm{~s}, 3 \mathrm{H}, \mathrm{CH}_{3}\right) \mathrm{ppm} .{ }^{13} \mathrm{C} \mathrm{NMR}\left(75 \mathrm{MHz}, \mathrm{CDCl}_{3}\right): \delta$ $=138.8\left(\mathrm{C}_{\text {quat }}\right), 138.0\left(\mathrm{C}_{\text {quat }}\right), 136.1\left(\mathrm{C}_{\text {quat }}\right), 129.2(2 \mathrm{Ar}-\mathrm{CH}), 129.1(2 \mathrm{Ar}-\mathrm{CH}), 128.6\left(\mathrm{C}_{\text {quat }}\right)$, 128.3 (Ar-CH), $128.1\left(\mathrm{C}_{\text {quat }}\right), 127.1(4 \mathrm{Ar}-\mathrm{CH}), 126.6(\mathrm{Ar}-\mathrm{CH}), 126.6(\mathrm{Ar}-\mathrm{CH}), 125.1\left(\mathrm{C}_{\text {quat }}\right)$, 122.0 (Ar-CH), 121.7 (Ar-CH), $114.3\left(\mathrm{C}_{\text {quat }}\right), 111.0(\mathrm{Ar}-\mathrm{CH}), 107.4\left(\mathrm{C}_{\text {quat }}\right), 49.2\left(\mathrm{CH}_{2}\right), 15.8$ $\left(\mathrm{CH}_{3}\right)$ ppm. HRMS (ESI): calcd. for $\mathrm{C}_{23} \mathrm{H}_{19} \mathrm{~N}_{2} \mathrm{~S}(\mathrm{M}+\mathrm{H})^{+} 355.12635$; found 355.12680.

1-Benzyl-3-(4-(trifluoromethyl)phenyl)-1H-indole-2-carbonitrile (9e): Brown liquid, 87\%, 272 g. ${ }^{1} \mathrm{H}$ NMR $\left(300 \mathrm{MHz}, \mathrm{CDCl}_{3}\right): \delta=7.86(\mathrm{~d}, J=8.5 \mathrm{~Hz}, 2 \mathrm{H}, \mathrm{Ar}-\mathrm{H}), 7.85(\mathrm{dd}, J=8.2 \mathrm{~Hz}, J$ $=0.9 \mathrm{~Hz}, 1 \mathrm{H}, \mathrm{Ar}-H), 7.79(\mathrm{~d}, J=8.5 \mathrm{~Hz}, 2 \mathrm{H}, \mathrm{Ar}-H), 7.45-7.42(\mathrm{~m}, 2 \mathrm{H}, \mathrm{Ar}-H), 7.38-7.28(\mathrm{~m}$, $4 \mathrm{H}, \mathrm{Ar}-H), 7.27-7.22(\mathrm{~m}, 2 \mathrm{H}, \mathrm{Ar}-\mathrm{H}), 5.55\left(\mathrm{~s}, 2 \mathrm{H}, \mathrm{CH}_{2}\right) \mathrm{ppm} .{ }^{13} \mathrm{C} \mathrm{NMR}\left(75 \mathrm{MHz}, \mathrm{CDCl}_{3}\right): \delta$ $=137.9\left(\mathrm{C}_{\text {quat }}\right), 135.8\left(\mathrm{C}_{\text {quat }}\right), 135.7\left(\mathrm{q}, J=1.2 \mathrm{~Hz}, \mathrm{C}_{\text {quat }}\right), 130.0\left(\mathrm{q}, J=32.7 \mathrm{~Hz}, \mathrm{C}_{\text {quat }}\right), 129.2$ (2Ar-CH), 129.1 (2Ar-CH), 128.4 (Ar-CH), $127.1(2 \mathrm{Ar}-\mathrm{CH}), 126.9(\mathrm{Ar}-\mathrm{CH}), 126.7\left(\mathrm{C}_{\text {quat }}\right), 126.1$ (q, $J=3.8 \mathrm{~Hz}, 2 \mathrm{Ar}-\mathrm{CH}), 124.9\left(\mathrm{C}_{\text {quat }}\right), 124.2\left(\mathrm{q}, J=272.2 \mathrm{~Hz}, \mathrm{C}_{\text {quat }}\right), 122.5(\mathrm{Ar}-\mathrm{CH}), 121.3$ (Ar-CH), $113.8\left(\mathrm{C}_{\text {quat }}\right), 111.2(\mathrm{Ar}-\mathrm{CH}), 108.1\left(\mathrm{C}_{\text {quat }}\right), 49.3\left(\mathrm{CH}_{2}\right) \mathrm{ppm} .{ }^{19} \mathrm{~F}$ NMR $(282 \mathrm{MHz}$, $\left.\mathrm{CDCl}_{3}\right): \delta=-62.5 \mathrm{ppm}$. HRMS (ESI): calcd. for $\mathrm{C}_{23} \mathrm{H}_{16} \mathrm{~F}_{3} \mathrm{~N}_{2}(\mathrm{M}+\mathrm{H})^{+} 377.12656$; found 377.12516.

1-Benzyl-3-(2-methoxyphenyl)-1H-indole-2-carbonitrile (9f): Brown solid, $89 \%, 250 \mathrm{mg}$. $\mathrm{mp} 132-134{ }^{\circ} \mathrm{C} .{ }^{1} \mathrm{H}$ NMR $\left(300 \mathrm{MHz}, \mathrm{CDCl}_{3}\right): \delta=7.91(\mathrm{dd}, J=8.2 \mathrm{~Hz}, J=0.9 \mathrm{~Hz}, 1 \mathrm{H}, \mathrm{Ar}-H)$, 7.52-7.40 (m, 4H, Ar- $H), 7.37-7.27(\mathrm{~m}, 5 \mathrm{H}, \mathrm{Ar}-H), 7.25-7.23(\mathrm{~m}, 2 \mathrm{H}, \mathrm{Ar}-H), 6.99$ (ddd, $J=$ $8.2 \mathrm{~Hz}, J=2.6 \mathrm{~Hz}, J=1.0 \mathrm{~Hz}, 1 \mathrm{H}, \mathrm{Ar}-\mathrm{H}), 5.54\left(\mathrm{~s}, 2 \mathrm{H}, \mathrm{CH}_{2}\right), 3.90\left(\mathrm{~s}, 3 \mathrm{H}, \mathrm{OCH}_{3}\right) \mathrm{ppm} .{ }^{13} \mathrm{C}$ $\operatorname{NMR}\left(75 \mathrm{MHz}, \mathrm{CDCl}_{3}\right): \delta=160.2\left(\mathrm{C}_{\text {quat }}\right), 138.0\left(\mathrm{C}_{\text {quat }}\right), 136.1\left(\mathrm{C}_{\text {quat }}\right), 133.2\left(\mathrm{C}_{\text {quat }}\right), 130.2$ (Ar-CH), 129.1 (2Ar-CH), $128.4\left(\mathrm{C}_{\text {quat }}\right), 128.3(\mathrm{Ar}-\mathrm{CH}), 127.0(2 \mathrm{Ar}-\mathrm{CH}), 126.6(\mathrm{Ar}-\mathrm{CH}), 125.2$ 
(C quat $), 122.0(\mathrm{Ar}-\mathrm{CH}), 121.8$ (Ar-CH), $121.3(\mathrm{Ar}-\mathrm{CH}), 114.2(\mathrm{Ar}-\mathrm{CH}), 114.1(\mathrm{Ar}-\mathrm{CH}), 111.0$ (Ar-CH), 109.1 ( $\left.\mathrm{C}_{\text {quat }}\right), 107.7\left(\mathrm{C}_{\text {quat }}\right), 55.5\left(\mathrm{CH}_{2}\right), 49.2\left(\mathrm{OCH}_{3}\right)$ ppm. HRMS (ESI): calcd. for $\mathrm{C}_{23} \mathrm{H}_{19} \mathrm{~N}_{2} \mathrm{O}(\mathrm{M}+\mathrm{H})^{+} 339.14919$; found 339.14959.

1-Benzyl-3-(2-fluorophenyl)-1H-indole-2-carbonitrile (9g): White solid, 92\%, $250 \mathrm{mg} . \mathrm{mp}$ 151-153 ${ }^{\circ} \mathrm{C} .{ }^{1} \mathrm{H}$ NMR $\left(300 \mathrm{MHz}, \mathrm{CDCl}_{3}\right): \delta=7.69(\mathrm{ddt}, J=8.2 \mathrm{~Hz}, J=2.2 \mathrm{~Hz}, J=1.0 \mathrm{~Hz}, 1 \mathrm{H}$, Ar-H), $7.62(\mathrm{~m}, 1 \mathrm{H}, \mathrm{Ar}-H), 7.48-7.39$ (m, 4H, Ar-H), 7.37-7.29 (m, 4H, Ar-H), 7.28-7.21 (m, $3 \mathrm{H}, \mathrm{Ar}-\mathrm{H}), 5.55\left(\mathrm{~s}, 2 \mathrm{H}, \mathrm{CH}_{2}\right)$ ppm. ${ }^{13} \mathrm{C} \mathrm{NMR}\left(75 \mathrm{MHz}, \mathrm{CDCl}_{3}\right): \delta=159.9(\mathrm{~d}, J=249.1 \mathrm{~Hz}$, $\left.\mathrm{C}_{\text {quat }}\right), 137.6$ ( $\left.\mathrm{C}_{\text {quat }}\right), 136.0$ (C $\left.\mathrm{C}_{\text {quat }}\right), 131.7(\mathrm{~d}, J=3.1 \mathrm{~Hz}, \mathrm{Ar}-\mathrm{CH}), 130.2(\mathrm{~d}, J=8.2 \mathrm{~Hz}, \mathrm{Ar}-\mathrm{CH})$, $129.1(2 \mathrm{Ar}-\mathrm{CH}), 128.3(\mathrm{Ar}-\mathrm{CH}), 127.1(2 \mathrm{Ar}-\mathrm{CH}), 126.5(\mathrm{Ar}-\mathrm{CH}), 125.7\left(\mathrm{C}_{\text {quat }}\right), 124.7(\mathrm{~d}, J=3.6$ $\mathrm{Hz}, \mathrm{Ar}-\mathrm{CH}), 122.0$ (d, J = 3.2 Hz, Ar-CH), $122.0(\mathrm{Ar}-\mathrm{CH}), 119.6$ (d, J = 15.4 Hz, $\left.\mathrm{C}_{\text {quat }}\right), 116.5$ $(\mathrm{d}, J=22.1 \mathrm{~Hz}, \mathrm{Ar}-\mathrm{CH}), 113.6\left(\mathrm{C}_{\text {quat }}\right), 111.9(\mathrm{Ar}-\mathrm{CH}), 109.1\left(\mathrm{C}_{\text {quat }}\right), 104.5\left(\mathrm{C}_{\text {quat }}\right), 49.4\left(\mathrm{CH}_{2}\right)$ ppm. ${ }^{19} \mathrm{~F} \mathrm{NMR}\left(282 \mathrm{MHz}, \mathrm{CDCl}_{3}\right): \delta=-112.1$ ppm. HRMS (ESI): calcd. for $\mathrm{C}_{22} \mathrm{H}_{16} \mathrm{FN}_{2}(\mathrm{M}$ $+\mathrm{H})^{+}$327.12975; found 327.13012.

1-Benzyl-3-(o-tolyl)-1H-indole-2-carbonitrile (9h): Brown solid, 92\%, $246 \mathrm{mg}$. mp 130-132 ${ }^{\circ} \mathrm{C} .{ }^{1} \mathrm{H}$ NMR $\left(300 \mathrm{MHz} \mathrm{CDCl}_{3}\right): \delta=7.49(\mathrm{dt}, J=8.1 \mathrm{~Hz}, J=1.0 \mathrm{~Hz}, 1 \mathrm{H}, \mathrm{Ar}-\mathrm{H}), 7.42-7.37(\mathrm{~m}$, 5H, Ar-H), 7.36-7.32 (m, 4H, Ar-H), 7.27-7.18 (m, 3H, Ar-H), 5.55 (s, 2H, CH $), 2.31(\mathrm{~s}, 3 \mathrm{H}$, $\left.\mathrm{CH}_{3}\right)$ ppm. ${ }^{13} \mathrm{C} \mathrm{NMR}\left(75 \mathrm{MHz}, \mathrm{CDCl}_{3}\right): \delta=137.5$ (C quat $), 137.3$ (C $\left.\mathrm{C}_{\text {quat }}\right), 136.2$ (C $\left.\mathrm{C}_{\text {quat }}\right), 131.0$ (Ar-CH), 130.9 (C quat), 130.7 (Ar-CH), 129.1 (2Ar-CH), 128.7 ( $\left.\mathrm{C}_{\text {quat }}\right), 128.6(\mathrm{Ar}-\mathrm{CH}), 128.2$ (Ar-CH), 127.0 (2Ar-CH), $126.4(\mathrm{Ar}-\mathrm{CH}), 126.3$ ( $\left.\mathrm{C}_{\text {quat }}\right), 126.0$ (Ar-CH), 122.0 (Ar-CH), 121.6 (Ar-CH), 113.8 ( $\left.\mathrm{C}_{\text {quat }}\right), 110.9(\mathrm{Ar}-\mathrm{CH}), 108.9\left(\mathrm{C}_{\text {quat }}\right), 49.2\left(\mathrm{CH}_{2}\right), 20.3\left(\mathrm{CH}_{3}\right) \mathrm{ppm}$. HRMS (ESI): calcd. for $\mathrm{C}_{23} \mathrm{H}_{19} \mathrm{~N}_{2}(\mathrm{M}+\mathrm{H})^{+}$323.15428; found 323.15463.

1-Benzyl-3-(3-methoxyphenyl)-1H-indole-2-carbonitrile (9i): Solid orange, 86\%, $241 \mathrm{mg}$. mp 131-133 ${ }^{\circ} \mathrm{C} .{ }^{1} \mathrm{H}$ NMR $\left(300 \mathrm{MHz}, \mathrm{CDCl}_{3}\right): \delta=7.91(\mathrm{~d}, J=8.2 \mathrm{~Hz}, 1 \mathrm{H}, \mathrm{Ar}-\mathrm{H}), 7.46(\mathrm{t}, J$ $=7.9 \mathrm{~Hz}, 1 \mathrm{H}, \mathrm{Ar}-H), 7.41-7.39(\mathrm{~m}, 2 \mathrm{H}, \mathrm{Ar}-H), 7.37-7.27(\mathrm{~m}, 5 \mathrm{H}, \mathrm{Ar}-H), 7.25-7.23(\mathrm{~m}, 2 \mathrm{H}$, Ar- $H), 6.99(\mathrm{ddd}, J=8.2 \mathrm{~Hz}, J=2.5 \mathrm{~Hz}, J=0.8 \mathrm{~Hz}, 1 \mathrm{H}, \mathrm{Ar}-H), 5.54\left(\mathrm{~s}, 2 \mathrm{H}, \mathrm{CH}_{2}\right), 3.90(\mathrm{~s}, 3 \mathrm{H}$, $\left.\mathrm{OCH}_{3}\right)$ ppm. ${ }^{13} \mathrm{C} \mathrm{NMR}\left(75 \mathrm{MHz}, \mathrm{CDCl}_{3}\right): \delta=160.2\left(\mathrm{C}_{\text {quat }}\right), 138.0\left(\mathrm{C}_{\text {quat }}\right), 136.1\left(\mathrm{C}_{\text {quat }}\right), 133.2$ ( $\left.\mathrm{C}_{\text {quat }}\right), 130.2(\mathrm{Ar}-\mathrm{CH}), 129.1(2 \mathrm{Ar}-\mathrm{CH}), 128.4\left(\mathrm{C}_{\text {quat }}\right), 128.3(\mathrm{Ar}-\mathrm{CH}), 127.1(2 \mathrm{Ar}-\mathrm{CH}), 126.6$ (Ar-CH), 125.2 ( $\left.\mathrm{C}_{\text {quat }}\right), 122.0(\mathrm{Ar}-\mathrm{CH}), 121.8(\mathrm{Ar}-\mathrm{CH}), 121.3(\mathrm{Ar}-\mathrm{CH}), 114.2\left(\mathrm{C}_{\text {quat }}\right), 114.2$ (Ar-CH), 114.1 (Ar-CH), $111.0(\mathrm{Ar}-\mathrm{CH}), 107.7\left(\mathrm{C}_{\text {quat }}\right), 55.5\left(\mathrm{CH}_{3}\right), 49.2\left(\mathrm{CH}_{2}\right) \mathrm{ppm}$. HRMS (ESI): calcd. for $\mathrm{C}_{23} \mathrm{H}_{19} \mathrm{~N}_{2} \mathrm{O}(\mathrm{M}+\mathrm{H})^{+} 339.14919$; found 339.14965.

1-Benzyl-3-(2,4-dimethoxyphenyl)-1H-indole-2-carbonitrile (9j): Brown liquid, 89\%, 248 mg. ${ }^{1} \mathrm{H} \mathrm{NMR}\left(300 \mathrm{MHz} \mathrm{CDCl}_{3}\right): \delta=7.64(\mathrm{dt}, J=8.1 \mathrm{~Hz}, J=0.9 \mathrm{~Hz}, 2 \mathrm{H}, \mathrm{Ar}-H), 7.42(\mathrm{~d}, J$ $=8.8 \mathrm{~Hz}, 1 \mathrm{H}, \mathrm{Ar}-H), 7.34-7.27(\mathrm{~m}, 5 \mathrm{H}, \mathrm{Ar}-H), 7.24-7.14(\mathrm{~m}, 3 \mathrm{H}, \mathrm{Ar}-H), 6.63(\mathrm{~s}, 1 \mathrm{H}, \mathrm{Ar}-\mathrm{H})$, $6.62(\mathrm{dd}, J=7.6 \mathrm{~Hz}, J=2.4 \mathrm{~Hz}, 1 \mathrm{H}, \mathrm{Ar}-\mathrm{H}), 5.49$ (s, 2H, $\left.\mathrm{CH}_{2}\right), 3.87$ (s, 3H, OCH $), 3.84$ (s, $\left.3 \mathrm{H}, \mathrm{OCH}_{3}\right) \mathrm{ppm} .{ }^{13} \mathrm{C} \mathrm{NMR}\left(75 \mathrm{MHz}, \mathrm{CDCl}_{3}\right): \delta=161.3\left(\mathrm{C}_{\text {quat }}\right), 158.1$ (C quat $), 137.6$ (C quat $)$,

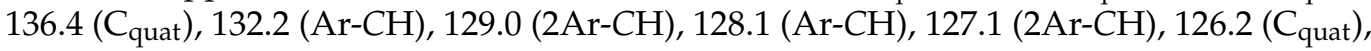
126.0 (Ar-CH), 124.7 (C quat), $122.4(\mathrm{Ar}-\mathrm{CH}), 121.3(\mathrm{Ar}-\mathrm{CH}), 114.3$ ( $\left.\mathrm{C}_{\text {quat }}\right), 113.3$ ( $\left.\mathrm{C}_{\text {quat }}\right), 110.7$ $(\mathrm{Ar}-\mathrm{CH}), 109.1\left(\mathrm{C}_{\text {quat }}\right), 104.9(\mathrm{Ar}-\mathrm{CH}), 99.2(\mathrm{Ar}-\mathrm{CH}), 55.6\left(\mathrm{OCH}_{3}\right), 55.4\left(\mathrm{OCH}_{3}\right), 49.2\left(\mathrm{CH}_{2}\right)$ ppm. HRMS (ESI): calcd. for $\mathrm{C}_{24} \mathrm{H}_{21} \mathrm{~N}_{2} \mathrm{O}_{2}(\mathrm{M}+\mathrm{H})^{+}$369.16030; found 369.16135.

1-Benzyl-3-(2,3-dihydrobenzo[b][1,4]dioxin-6-yl)-1H-indole-2-carbonitrile (9k): Brown solid, 90\%, 273 mg. mp 140-142 ${ }^{\circ} \mathrm{C} .{ }^{1} \mathrm{H}$ NMR (300 MHz, $\left.\mathrm{CDCl}_{3}\right): \delta=7.87(\mathrm{~d}, J=8.2 \mathrm{~Hz}, 2 \mathrm{H}$, Ar-H), 7.38-7.36 (m, 2H, Ar-H), 7.35-7.27 (m, 4H, Ar-H), 7.25-7.21 (m, 4H, Ar-H), 7.02 (d, $J=8.3 \mathrm{~Hz}, 2 \mathrm{H}, \mathrm{Ar}-\mathrm{H}), 5.51\left(\mathrm{~s}, 2 \mathrm{H}, \mathrm{CH}_{2}\right), 4.32\left(\mathrm{~s}, 4 \mathrm{H}, 2 \times \mathrm{CH}_{2}\right) \mathrm{ppm} .{ }^{13} \mathrm{C} \mathrm{NMR}(75 \mathrm{MHz}$, $\left.\mathrm{CDCl}_{3}\right): \delta=144.0$ (C $\left.\mathrm{C}_{\text {quat }}\right), 143.7$ (C quat $), 137.8$ ( $\left.\mathrm{C}_{\text {quat }}\right), 136.1$ (C quat $), 129.0$ (2Ar-CH), 128.1 $(\mathrm{Ar}-\mathrm{CH}), 128.1\left(\mathrm{C}_{\text {quat }}\right), 127.0(2 \mathrm{Ar}-\mathrm{CH}), 126.4(\mathrm{Ar}-\mathrm{CH}), 125.1$ (2 $\left.\mathrm{C}_{\text {quat }}\right), 122.0(\mathrm{Ar}-\mathrm{CH}), 121.8$ (Ar-CH), 121.7 (Ar-CH), 118.0 (Ar-CH), 117.6 (Ar-CH), 114.3 (C quat), 110.8 (Ar-CH), 107.2 (C $\left.\mathrm{C}_{\text {quat }}\right), 64.5\left(\mathrm{CH}_{2}\right), 64.4\left(\mathrm{CH}_{2}\right), 49.0\left(\mathrm{CH}_{2}\right)$ ppm. HRMS (ESI): calcd. for $\mathrm{C}_{24} \mathrm{H}_{19} \mathrm{~N}_{2} \mathrm{O}_{2}(\mathrm{M}+$ $\mathrm{H})^{+}$367.14465; found 367.14425.

1-Benzyl-3-(6-methoxynaphthalen-2-yl)-1H-indole-2-carbonitrile (91): Brown solid, 84\%, 272 mg. mp 147-149 ${ }^{\circ} \mathrm{C} .{ }^{1} \mathrm{H}$ NMR $\left(300 \mathrm{MHz}, \mathrm{CDCl}_{3}\right): \delta=8.13$ (s, 1H, Ar-H), 7.96-7.81 (m, 4H, Ar-H), 7.41-7.19 (m, 10H, Ar-H), $5.52\left(\mathrm{~s}, 2 \mathrm{H}, \mathrm{CH}_{2}\right), 3.94$ (s, 3H, OCH $\left.{ }_{3}\right)$ ppm. ${ }^{13} \mathrm{C} \mathrm{NMR}$ $\left(75 \mathrm{MHz}, \mathrm{CDCl}_{3}\right): \delta=158.3\left(\mathrm{C}_{\text {quat }}\right), 138.0\left(\mathrm{C}_{\text {quat }}\right), 136.2\left(\mathrm{C}_{\text {quat }}\right), 134.3\left(\mathrm{C}_{\text {quat }}\right), 129.8(\mathrm{Ar}-\mathrm{CH})$, $129.2\left(\mathrm{C}_{\text {quat }}\right), 129.1(2 \mathrm{Ar}-\mathrm{CH}), 128.7$ ( $\left.\mathrm{C}_{\text {quat }}\right), 128.2(\mathrm{Ar}-\mathrm{CH}), 127.8$ (Ar-CH), $127.7(\mathrm{Ar}-\mathrm{CH})$, 127.2 (Ar-CH), 127.1 ( $\left.\mathrm{C}_{\text {quat }}\right), 127.0(2 \mathrm{Ar}-\mathrm{CH}), 126.6(\mathrm{Ar}-\mathrm{CH}), 125.4\left(\mathrm{C}_{\text {quat }}\right), 121.9(\mathrm{Ar}-\mathrm{CH})$, 
121.8 (Ar-CH), 119.5 (Ar-CH), 114.4 (C quat $), 111.0(\mathrm{Ar}-\mathrm{CH}), 107.6\left(\mathrm{C}_{\text {quat }}\right), 105.8(\mathrm{Ar}-\mathrm{CH}), 55.5$ $\left(\mathrm{OCH}_{3}\right), 49.2\left(\mathrm{CH}_{2}\right)$ ppm. HRMS (ESI): calcd. for $\mathrm{C}_{27} \mathrm{H}_{21} \mathrm{~N}_{2} \mathrm{O}(\mathrm{M}+\mathrm{H})^{+}$389.16484; found 389.16527.

1-Benzyl-3-(2-methoxypyridin-3-yl)-1H-indole-2-carbonitrile (9m): Brown liquid, 86\%, 241 mg. ${ }^{1} \mathrm{H} \mathrm{NMR}\left(300 \mathrm{MHz} \mathrm{CDCl}_{3}\right): \delta=8.27(\mathrm{dd}, J=5.0 \mathrm{~Hz}, J=1.9 \mathrm{~Hz}, 1 \mathrm{H}, \mathrm{Ar}-\mathrm{H}), 7.83(\mathrm{dd}$, $J=7.3 \mathrm{~Hz}, J=1.9 \mathrm{~Hz}, 1 \mathrm{H}, \mathrm{Ar}-H), 7.63(\mathrm{~d}, J=8.2 \mathrm{~Hz}, 2 \mathrm{H}, \mathrm{Ar}-H), 7.39-7.37$ (m, 3H, Ar-H), 7.34-7.29 (m, 3H, Ar-H), 7.27-7.19 (m, 2H, Ar-H), $7.05(\mathrm{dd}, J=7.3 \mathrm{~Hz}, J=5.1 \mathrm{~Hz}, 1 \mathrm{H}, \mathrm{Ar}-H)$, $5.54\left(\mathrm{~s}, 2 \mathrm{H}, \mathrm{CH}_{2}\right), 4.03\left(\mathrm{~s}, 3 \mathrm{H}, \mathrm{OCH}_{3}\right) \mathrm{ppm} .{ }^{13} \mathrm{C} \mathrm{NMR}\left(75 \mathrm{MHz}, \mathrm{CDCl}_{3}\right): \delta=161.3\left(\mathrm{C}_{\text {quat }}\right)$, $147.0(\mathrm{Ar}-\mathrm{CH}), 139.9(\mathrm{Ar}-\mathrm{CH}), 137.6\left(\mathrm{C}_{\text {quat }}\right), 136.1\left(\mathrm{C}_{\text {quat }}\right), 129.1(2 \mathrm{Ar}-\mathrm{CH}), 128.3(\mathrm{Ar}-\mathrm{CH})$, $127.1(2 \mathrm{Ar}-\mathrm{CH}), 126.4(\mathrm{Ar}-\mathrm{CH}), 125.8$ (C quat), 122.8 ( $\left.\mathrm{C}_{\text {quat }}\right), 122.1(\mathrm{Ar}-\mathrm{CH}), 121.8(\mathrm{Ar}-\mathrm{CH})$, 117.0 (Ar-CH), 115.3 ( $\left.\mathrm{C}_{\text {quat }}\right), 113.7$ (C quat $), 111.0$ (Ar- $\left.\mathrm{CH}\right), 109.4$ ( $\left.\mathrm{C}_{\text {quat }}\right), 53.6\left(\mathrm{CH}_{2}\right), 49.4$ $\left(\mathrm{OCH}_{3}\right)$ ppm. HRMS (ESI): calcd. for $\mathrm{C}_{22} \mathrm{H}_{18} \mathrm{~N}_{3} \mathrm{O}(\mathrm{M}+\mathrm{H})^{+} 340.14499$; found 340.14471 .

1-Benzyl-5-methoxy-3-(4-methoxyphenyl)-1H-indole-2-carbonitrile (9n): Brown solid, 89\%, $254 \mathrm{mg} . \mathrm{mp} 152-154{ }^{\circ} \mathrm{C} .{ }^{1} \mathrm{H}$ NMR $\left(300 \mathrm{MHz} \mathrm{CDCl}_{3}\right): \delta=7.65(\mathrm{~d}, J=8.8 \mathrm{~Hz}, 2 \mathrm{H}, \mathrm{Ar}-H)$, 7.37-7.27 (m, 3H, Ar-H), 7.26-7.18 (m, 4H, Ar-H), 7.10-7.02 (m, 3H, Ar-H), 5.48 (s, 2H, CH $)$, $3.89\left(\mathrm{~s}, 3 \mathrm{H}, \mathrm{OCH}_{3}\right), 3.82\left(\mathrm{~s}, 3 \mathrm{H}, \mathrm{OCH}_{3}\right) \mathrm{ppm} .{ }^{13} \mathrm{C} \mathrm{NMR}\left(75 \mathrm{MHz}, \mathrm{CDCl}_{3}\right): \delta=159.5\left(\mathrm{C}_{\text {quat }}\right)$, 155.7 (C quat $), 136.3$ ( $\left.\mathrm{C}_{\text {quat }}\right), 133.3$ (C quat $), 129.9$ (2Ar-CH), $129.1(2 \mathrm{Ar}-\mathrm{CH}), 128.2(\mathrm{Ar}-\mathrm{CH})$, 127.6 (C quat), 127.0 (2Ar-CH), 125.6 (C quat), 124.5 ( $\left.\mathrm{C}_{\text {quat }}\right), 118.0(\mathrm{Ar}-\mathrm{CH}), 114.7(2 \mathrm{Ar}-\mathrm{CH})$, 114.5 (C quat $), 111.9(\mathrm{Ar}-\mathrm{CH}), 107.5$ (C quat $), 101.6(\mathrm{Ar}-\mathrm{CH}), 55.9\left(\mathrm{OCH}_{3}\right), 55.5\left(\mathrm{OCH}_{3}\right), 49.3$ $\left(\mathrm{CH}_{2}\right)$ ppm. HRMS (ESI): calcd. for $\mathrm{C}_{24} \mathrm{H}_{21} \mathrm{~N}_{2} \mathrm{O}_{2}(\mathrm{M}+\mathrm{H})^{+}$369.15975; found 369.16033.

1-Benzyl-6-methoxy-3-(3-methoxyphenyl)-1H-indole-2-carbonitrile (9o): Brown solid, 93\%, $264 \mathrm{mg} . \mathrm{mp} 150-152{ }^{\circ} \mathrm{C} .{ }^{1} \mathrm{H}$ NMR $\left(300 \mathrm{MHz} \mathrm{CDCl}_{3}\right): \delta=7.78(\mathrm{~d}, J=8.9 \mathrm{~Hz}, 1 \mathrm{H}, \mathrm{Ar}-H)$, $7.46(\mathrm{t}, J=7.9 \mathrm{~Hz}, 1 \mathrm{H}, \mathrm{Ar}-H), 7.41-7.22(\mathrm{~m}, 7 \mathrm{H}, \mathrm{Ar}-H), 6.99(\mathrm{dd}, J=7.9 \mathrm{~Hz}, J=2.1 \mathrm{~Hz}$, $1 \mathrm{H}, \mathrm{Ar}-H), 6.93(\mathrm{dd}, J=8.9 \mathrm{~Hz}, J=2.1 \mathrm{~Hz}, 1 \mathrm{H}, \mathrm{Ar}-H), 6.75(\mathrm{~d}, J=2.1 \mathrm{~Hz}, 1 \mathrm{H}, \mathrm{Ar}-H), 5.49$ $\left(\mathrm{s}, 2 \mathrm{H}, \mathrm{CH}_{2}\right), 3.91\left(\mathrm{~s}, 3 \mathrm{H}, \mathrm{OCH}_{3}\right), 3.84\left(\mathrm{~s}, 3 \mathrm{H}, \mathrm{OCH}_{3}\right) \mathrm{ppm} .{ }^{13} \mathrm{C} \mathrm{NMR}\left(75 \mathrm{MHz}, \mathrm{CDCl}_{3}\right)$ : $\delta=160.2$ ( $\left.\mathrm{C}_{\text {quat }}\right), 159.8$ ( $\left.\mathrm{C}_{\text {quat }}\right), 139.1$ ( $\left.\mathrm{C}_{\text {quat }}\right), 136.1$ ( $\left.\mathrm{C}_{\text {quat }}\right), 133.3$ ( $\left.\mathrm{C}_{\text {quat }}\right), 130.2(\mathrm{Ar}-\mathrm{CH})$, 129.1 (2Ar-CH), 128.8 ( $\left.\mathrm{C}_{\text {quat }}\right), 128.2(\mathrm{Ar}-\mathrm{CH}), 127.0(2 \mathrm{Ar}-\mathrm{CH}), 122.6(\mathrm{Ar}-\mathrm{CH}), 121.2(\mathrm{Ar}-\mathrm{CH})$, $119.5\left(\mathrm{C}_{\text {quat }}\right), 114.6\left(\mathrm{C}_{\text {quat }}\right), 114.2(\mathrm{Ar}-\mathrm{CH}), 114.0(\mathrm{Ar}-\mathrm{CH}), 113.1(\mathrm{Ar}-\mathrm{CH}), 106.4\left(\mathrm{C}_{\text {quat }}\right), 93.1$ $(\mathrm{Ar}-\mathrm{CH}), 55.7\left(\mathrm{OCH}_{3}\right), 55.5\left(\mathrm{OCH}_{3}\right), 49.1\left(\mathrm{CH}_{2}\right)$ ppm. HRMS (ESI): calcd. for $\mathrm{C}_{24} \mathrm{H}_{21} \mathrm{~N}_{2} \mathrm{O}_{2}$ $(\mathrm{M}+\mathrm{H})^{+}$369.15975; found 369.16017.

1-Benzyl-5-fluoro-3-(3-methoxyphenyl)-1H-indole-2-carbonitrile (9p): Yellow viscous liquid, 98\%, $279 \mathrm{mg} .{ }^{1} \mathrm{H}$ NMR (300 MHz, $\left.\mathrm{CDCl}_{3}\right): \delta=7.51(\mathrm{dd}, J=7.4 \mathrm{~Hz}, J=1.6 \mathrm{~Hz}, 1 \mathrm{H})$, $7.44(\mathrm{td}, J=8.1 \mathrm{~Hz}, J=1.7 \mathrm{~Hz}, 1 \mathrm{H}), 7.36-7.23(\mathrm{~m}, 1 \mathrm{H}), 7.16-7.08(\mathrm{~m}, 3 \mathrm{H}), 5.52(\mathrm{~s}, 2 \mathrm{H}), 3.90$ (s, 3H) ppm. ${ }^{13} \mathrm{C} \mathrm{NMR}\left(75 \mathrm{MHz}, \mathrm{CDCl}_{3}\right): \delta=158.7\left(\mathrm{~d}, J=238.7 \mathrm{~Hz}, \mathrm{C}_{\text {quat }}\right), 156.9$ (C quat), 136.0 ( $\left.\mathrm{C}_{\text {quat }}\right), 134.1$ ( $\left.\mathrm{C}_{\text {quat }}\right), 131.4(\mathrm{Ar}-\mathrm{CH}), 130.0(\mathrm{Ar}-\mathrm{CH}), 129.1(2 \mathrm{Ar}-\mathrm{CH}), 128.3(\mathrm{Ar}-\mathrm{CH})$, $127.0(2 \mathrm{Ar}-\mathrm{CH}), 126.4\left(\mathrm{~d}, J=10.5 \mathrm{~Hz}, \mathrm{C}_{\text {quat }}\right), 124.2\left(\mathrm{~d}, J=5.5 \mathrm{~Hz}, \mathrm{C}_{\text {quat }}\right), 121.0(\mathrm{Ar}-\mathrm{CH})$, 120.1 ( $\left.\mathrm{C}_{\text {quat }}\right), 115.2(\mathrm{~d}, J=26.8 \mathrm{~Hz}, \mathrm{Ar}-\mathrm{CH}), 113.7$ (C quat $), 111.8,(\mathrm{~d}, J=9.4 \mathrm{~Hz}, \mathrm{Ar}-\mathrm{CH}), 111.5$ $(\mathrm{Ar}-\mathrm{CH}), 110.8\left(\mathrm{C}_{\text {quat }}\right), 106.8(\mathrm{~d}, \mathrm{~J}=24.1 \mathrm{~Hz}, \mathrm{Ar}-\mathrm{CH}), 55.4\left(\mathrm{OCH}_{3}\right), 49.5\left(\mathrm{CH}_{2}\right) \mathrm{ppm} .{ }^{19} \mathrm{~F}$ NMR (282 MHz, $\left.\mathrm{CDCl}_{3}\right): \delta=-121.7 \mathrm{ppm}$. HRMS (ESI): calcd. for $\mathrm{C}_{23} \mathrm{H}_{18} \mathrm{FN}_{2} \mathrm{O}(\mathrm{M}+\mathrm{H})^{+}$ 357.14032; found 357.14081.

\subsubsection{General Procedure for Heck Coupling (Series 10)}

Compound 7a (300 mg, $0.083 \mathrm{mmol}, 1$ equiv.), alkene (1.2 mmol, 1.5 equiv), KOAc (50 mg, $0.5 \mathrm{mmol}, 6$ equiv.), $\mathrm{Pd}(\mathrm{OAc})_{2}\left(0.74 \mathrm{mg}, 0.003 \mathrm{mmol}, 0.04\right.$ equiv.), $\mathrm{tBuNH} \mathrm{Cl}_{4}(46 \mathrm{mg}$, $0.166 \mathrm{mmol}, 2$ equiv. $)$ and DMF $(5 \mathrm{~mL})$ were introduced in a sealed tube. The tube was vacuumed and backfilled with argon. The reaction mixture was stirred at $80^{\circ} \mathrm{C}$ for $24 \mathrm{~h}$ and then cooled to room temperature. The reaction mixture was quenched with water, filtered through a Celite ${ }^{\complement}$ pad and washed with EtOAc $(100 \mathrm{~mL})$. Then, the resulting organic layer was washed with sat. aq. $\mathrm{NH}_{4} \mathrm{Cl}(2 \times 40 \mathrm{~mL})$ and brine $(3 \times 40 \mathrm{~mL})$. The organic layers were dried over $\mathrm{MgSO}_{4}$ and concentrated under reduced pressure. The crude product was purified by column chromatography using petroleum ether/EtOAc as eluent (70:30).

(E)-Methyl-3-(1-benzyl-2-cyano-1H-indol-3-yl) acrylate (10a): Brown solid, 92\%, $244 \mathrm{mg}$. ${ }^{1} \mathrm{H} \mathrm{NMR}\left(300 \mathrm{MHz} \mathrm{CDCl}_{3}\right): \delta=7.99(\mathrm{~d}, J=16.2 \mathrm{~Hz}, 1 \mathrm{H}, \mathrm{CH}=\mathrm{CH}), 7.95(\mathrm{dt}, J=8.2 \mathrm{~Hz}, J$ $=0.9 \mathrm{~Hz}, 1 \mathrm{H}, \mathrm{Ar}-H), 7.46-7.30(\mathrm{~m}, 6 \mathrm{H}, \mathrm{Ar}-H), 7.18(\mathrm{~m}, 2 \mathrm{H}, \mathrm{Ar}-H), 6.76(\mathrm{~d}, J=16.2 \mathrm{~Hz}, 1 \mathrm{H}$, 
$\mathrm{CH}=\mathrm{CH}), 5.50\left(\mathrm{~s}, 2 \mathrm{H}, \mathrm{CH}_{2}\right), 3.85\left(\mathrm{~s}, 3 \mathrm{H}, \mathrm{OCH}_{3}\right)$ ppm. ${ }^{13} \mathrm{C} \mathrm{NMR}\left(75 \mathrm{MHz} \mathrm{CDCl}_{3}\right): \delta=167.5$ ( $\left.\mathrm{C}_{\text {quat }}\right), 138.1$ ( $\left.\mathrm{C}_{\text {quat }}\right), 135.3$ ( $\left.\mathrm{C}_{\text {quat }}\right), 134.0(\mathrm{CH}=\mathrm{CH}), 129.2(2 \mathrm{Ar}-\mathrm{CH}), 128.5(\mathrm{Ar}-\mathrm{CH}), 127.1$ (Ar-CH), 127.0 (2Ar-CH), 124.9 (C quat $), 123.2$ (Ar-CH), 121.8 (C quat), 121.6 (Ar-CH), 119.1 $(\mathrm{CH}=\mathrm{CH}), 112.8\left(\mathrm{C}_{\text {quat }}\right), 111.7\left(\mathrm{C}_{\text {quat }}\right), 111.5(\mathrm{Ar}-\mathrm{CH}), 52.0\left(\mathrm{OCH}_{3}\right), 49.6\left(\mathrm{CH}_{2}\right) \mathrm{ppm} . \mathrm{HRMS}$ (ESI): calcd. for $\mathrm{C}_{20} \mathrm{H}_{17} \mathrm{~N}_{2} \mathrm{O}_{2}(\mathrm{M}+\mathrm{H})^{+} 317.12900$; found 317.12946.

(E)-1-Benzyl-3-styryl-1H-indole-2-carbonitrile (10b): Brown solid, 89\%, $257 \mathrm{mg} .{ }^{1} \mathrm{H}$ $\operatorname{NMR}\left(300 \mathrm{MHz}, \mathrm{CDCl}_{3}\right): \delta=8.06(\mathrm{dt}, J=8.1 \mathrm{~Hz}, J=0.7 \mathrm{~Hz}, 1 \mathrm{H}), 7.66-7.58(\mathrm{~m}, 2 \mathrm{H}, \mathrm{Ar}-\mathrm{H})$, $7.51(\mathrm{~d}, J=16.5 \mathrm{~Hz}, \mathrm{CH}=\mathrm{CH}), 7.45-7.29(\mathrm{~m}, 10 \mathrm{H}, 9 \mathrm{Ar}-\mathrm{H}+\mathrm{CH}=\mathrm{CH}), 7.23-7.20(\mathrm{~m}, 2 \mathrm{H}, \mathrm{Ar}-H)$, 5.45 (s, 2H, $\left.\mathrm{CH}_{2}\right)$ ppm. ${ }^{13} \mathrm{C} \mathrm{NMR}\left(75 \mathrm{MHz}, \mathrm{CDCl}_{3}\right): \delta=138.1\left(\mathrm{C}_{\text {quat }}\right), 137.3\left(\mathrm{C}_{\text {quat }}\right), 135.9$ ( $\left.\mathrm{C}_{\text {quat }}\right), 131.2(\mathrm{CH}=\mathrm{CH}), 129.1(2 \mathrm{Ar}-\mathrm{CH}), 128.9(2 \mathrm{ArCH}), 128.2(\mathrm{Ar}-\mathrm{CH}), 128.1(\mathrm{Ar}-\mathrm{CH}), 126.9$ (2Ar-CH), $126.6(\mathrm{Ar}-\mathrm{CH}), 126.5(2 \mathrm{Ar}-\mathrm{CH}), 125.2$ ( $\left.\mathrm{C}_{\text {quat }}\right), 124.6$ ( $\left.\mathrm{C}_{\text {quat }}\right), 122.0(\mathrm{CH}=\mathrm{CH}), 121.7$ $(\mathrm{Ar}-\mathrm{CH}), 118.4(\mathrm{Ar}-\mathrm{CH}), 113.9\left(\mathrm{C}_{\text {quat }}\right), 111.0(\mathrm{Ar}-\mathrm{CH}), 108.6\left(\mathrm{C}_{\text {quat }}\right), 49.1\left(\mathrm{CH}_{2}\right) \mathrm{ppm}$. HRMS (ESI): calcd. for $\mathrm{C}_{24} \mathrm{H}_{19} \mathrm{~N}_{2}(\mathrm{M}+\mathrm{H})^{+}$335.15482; found 335.15532.

1-Benzyl-3-(3-cyanoprop-1-en-1-yl)-1H-indole-2-carbonitrile (10c): Brown solid, 81\%, $192 \mathrm{mg} .{ }^{1} \mathrm{H}$ NMR (300 MHz, $\left.\mathrm{CDCl}_{3}\right): \delta=8.09$ (d, J = 8.1 Hz, 1H, Z-isomer), 7.79 (d, J $=8.2 \mathrm{~Hz}, 1 \mathrm{H}$, E-isomer), $7.61(\mathrm{~d}, J=16.7 \mathrm{~Hz}, 1 \mathrm{H}$, E-isomer $), 7.52-7.29(\mathrm{~m}, \mathrm{Ar}-\mathrm{CH}), 7.23-7.18$ (m, Ar-CH), $6.14(\mathrm{~d}, J=16.7 \mathrm{~Hz}, 1 \mathrm{H}, E$-isomer), $5.64(\mathrm{~d}, J=12.1 \mathrm{~Hz}, 1 \mathrm{H}, \mathrm{Z}$-isomer $), 5.48(\mathrm{~s}, 2 \mathrm{H})$ ppm. ${ }^{13} \mathrm{C}$ NMR E-isomer $\left(75 \mathrm{MHz}, \mathrm{CDCl}_{3}\right): \delta=139.5(\mathrm{CH}=\mathrm{CH}), 137.9\left(\mathrm{C}_{\text {quat }}\right), 134.9\left(\mathrm{C}_{\text {quat }}\right)$, 129.2 (2Ar-CH), 128.6 (Ar-CH), $127.4(\mathrm{Ar}-\mathrm{CH}), 126.9$ (2Ar- $\mathrm{CH}), 124.1\left(\mathrm{C}_{\text {quat }}\right), 123.7(\mathrm{Ar}-\mathrm{CH})$, 120.8 (Ar-CH), 120.7 ( $\left.\mathrm{C}_{\text {quat }}\right), 118.3$ ( $\left.\mathrm{C}_{\text {quat }}\right), 111.2$ ( $\left.\mathrm{C}_{\text {quat }}\right), 111.7(\mathrm{Ar}-\mathrm{CH}), 111.3$ ( $\left.\mathrm{C}_{\text {quat }}\right), 96.9$ $(\mathrm{CH}=\mathrm{CH}), 49.6\left(\mathrm{CH}_{2}\right)$ ppm. HRMS (ESI): calcd. for $\mathrm{C}_{19} \mathrm{H}_{14} \mathrm{~N}_{3}(\mathrm{M}+\mathrm{H})^{+}$284.11877; found 284.11842.

(E)-Methyl-3-(1-benzyl-2-cyano-5-methoxy-1H-indol-3-yl) acrylate (10d): Brown solid, 88\%, 236 mg. ${ }^{1} \mathrm{H}$ NMR $\left(300 \mathrm{MHz} \mathrm{CDCl}_{3}\right): \delta=7.97(\mathrm{~d}, J=16.2 \mathrm{~Hz}, 1 \mathrm{H}, \mathrm{CH}=\mathrm{CH}), 7.36-7.28$ $(\mathrm{m}, 3 \mathrm{H}, \mathrm{Ar}-\mathrm{CH}), 7.27-7.23(\mathrm{~m}, 2 \mathrm{H}, \mathrm{Ar}-\mathrm{CH}), 7.16(\mathrm{dd}, J=7.5 \mathrm{~Hz}, J=2.0 \mathrm{~Hz}, 2 \mathrm{H}, \mathrm{Ar}-\mathrm{CH})$, $7.06(\mathrm{dd}, J=9.2 \mathrm{~Hz}, J=2.3 \mathrm{~Hz}, 1 \mathrm{H}, \mathrm{Ar}-\mathrm{CH}), 6.69(\mathrm{~d}, J=16.2 \mathrm{~Hz}, 1 \mathrm{H}, \mathrm{CH}=\mathrm{CH}) 5.46(\mathrm{~s}, 2 \mathrm{H}$, $\left.\mathrm{CH}_{2}\right), 3.88\left(\mathrm{~s}, 3 \mathrm{H}, \mathrm{OCH}_{3}\right), 3.85\left(\mathrm{~s}, 3 \mathrm{H}, \mathrm{OCH}_{3}\right)$ ppm. ${ }^{13} \mathrm{C} \mathrm{NMR}\left(75 \mathrm{MHz}, \mathrm{CDCl}_{3}\right): \delta=167.6$ ( $\left.\mathrm{C}_{\text {quat }}\right), 156.6$ ( $\left.\mathrm{C}_{\text {quat }}\right), 135.3$ ( $\left.\mathrm{C}_{\text {quat }}\right), 134.1(\mathrm{CH}=\mathrm{CH}), 133.2\left(\mathrm{C}_{\text {quat }}\right), 129.2(2 \mathrm{Ar}-\mathrm{CH}), 128.5$ (Ar-CH), 126.9 (2Ar-CH), 125.5 (C quat $), 120.8$ ( $\left.\mathrm{C}_{\text {quat }}\right), 118.4(\mathrm{Ar}-\mathrm{CH}), 118.1(\mathrm{Ar}-\mathrm{CH}), 112.9$ (C quat), $112.4(\mathrm{Ar}-\mathrm{CH}), 111.3\left(\mathrm{C}_{\text {quat }}\right), 101.6(\mathrm{Ar}-\mathrm{CH}), 55.9\left(\mathrm{OCH}_{3}\right), 51.9\left(\mathrm{OCH}_{3}\right), 49.7\left(\mathrm{CH}_{2}\right)$ ppm. HRMS (ESI): calcd. for $\mathrm{C}_{21} \mathrm{H}_{19} \mathrm{~N}_{2} \mathrm{O}_{3}(\mathrm{M}+\mathrm{H})^{+}$347.13957; found 347.14053.

(E)-Methyl-3-(1-benzyl-2-cyano-6-methoxy-1H-indol-3-yl) acrylate (10e): Brown solid, 92\%, $247 \mathrm{mg} .{ }^{1} \mathrm{H}$ NMR $\left(300 \mathrm{MHz}, \mathrm{CDCl}_{3}\right): \delta=7.90(\mathrm{~d}, J=16.1 \mathrm{~Hz}, 1 \mathrm{H}, \mathrm{CH}=\mathrm{CH}), 7.76(\mathrm{~d}, J=$ $9.0 \mathrm{~Hz}, 1 \mathrm{H}, \mathrm{Ar}-\mathrm{CH}), 7.35-7.28$ (m, 3H, Ar-CH), 7.21-7.13 (m, 2H, Ar-CH), 6.94 (dd, J = 8.9 $\mathrm{Hz}, J=2.0 \mathrm{~Hz}, 1 \mathrm{H}, \mathrm{Ar}-\mathrm{CH}), 6.71(\mathrm{~s}, 1 \mathrm{H}, \mathrm{Ar}-\mathrm{CH}), 6.68(\mathrm{~d}, J=16.1 \mathrm{~Hz}, 1 \mathrm{H}, \mathrm{CH}=\mathrm{CH}), 5.40$ (s, $\left.2 \mathrm{H}, \mathrm{CH}_{2}\right), 3.83\left(\mathrm{~s}, 3 \mathrm{H}, \mathrm{OCH}_{3}\right), 3.80\left(\mathrm{~s}, 3 \mathrm{H}, \mathrm{OCH}_{3}\right) \mathrm{ppm} .{ }^{13} \mathrm{C} \mathrm{NMR}\left(75 \mathrm{MHz}, \mathrm{CDCl}_{3}\right): \delta=$ 167.4 ( $\left.\mathrm{C}_{\text {quat }}\right), 159.9$ ( $\left.\mathrm{C}_{\text {quat }}\right), 139.3$ ( $\left.\mathrm{C}_{\text {quat }}\right), 135.2$ ( $\left.\mathrm{C}_{\text {quat }}\right), 134.6\left(\mathrm{C}_{\text {quat }}\right), 133.9(\mathrm{CH}=\mathrm{CH}), 129.2$ (2Ar-CH), $128.4(\mathrm{Ar}-\mathrm{CH}), 126.9(2 \mathrm{Ar}-\mathrm{CH}), 122.2(\mathrm{Ar}-\mathrm{CH}), 122.0\left(\mathrm{C}_{\text {quat }}\right), 118.8(\mathrm{Ar}-\mathrm{CH}), 113.9$ $(\mathrm{Ar}-\mathrm{CH}), 113.0\left(\mathrm{C}_{\text {quat }}\right), 110.4\left(\mathrm{C}_{\text {quat }}\right), 93.6(\mathrm{CH}=\mathrm{CH}), 55.6\left(\mathrm{OCH}_{3}\right), 51.8\left(\mathrm{OCH}_{3}\right), 49.3\left(\mathrm{CH}_{2}\right)$ ppm. HRMS (ESI): calcd. for $\mathrm{C}_{21} \mathrm{H}_{19} \mathrm{~N}_{2} \mathrm{O}_{3}(\mathrm{M}+\mathrm{H})^{+}$347.13957; found 347.14046.

(E)-Methyl-3-(1-benzyl-2-cyano-5-fluoro-1H-indol-3-yl)acrylate (10f): Brown solid, 81\%, $215 \mathrm{mg} .{ }^{1} \mathrm{H} \mathrm{NMR}\left(300 \mathrm{MHz}, \mathrm{CDCl}_{3}\right): \delta=7.92(\mathrm{~d}, J=16.2 \mathrm{~Hz}, 1 \mathrm{H}, \mathrm{CH}=\mathrm{CH}), 7.57$ (dd, J = 9.1 $\mathrm{Hz}, \mathrm{J}=2.4 \mathrm{~Hz}, 1 \mathrm{H}, \mathrm{Ar}-\mathrm{CH}), 7.40-7.27(\mathrm{~m}, 4 \mathrm{H}, \mathrm{Ar}-\mathrm{CH}), 7.22-7.12(\mathrm{~m}, 3 \mathrm{H}, \mathrm{Ar}-\mathrm{CH}), 6.67$ (d, J $=16.2 \mathrm{~Hz}, 1 \mathrm{H}, \mathrm{CH}=\mathrm{CH}), 5.48\left(\mathrm{~s}, 2 \mathrm{H}, \mathrm{CH}_{2}\right), 3.85\left(\mathrm{~s}, 3 \mathrm{H}, \mathrm{OCH}_{3}\right) \mathrm{ppm} .{ }^{13} \mathrm{C} \mathrm{NMR}(75 \mathrm{MHz}$, $\left.\mathrm{CDCl}_{3}\right): \delta=167.3\left(\mathrm{C}_{\text {quat }}\right), 159.6\left(\mathrm{~d}, J=241.4 \mathrm{~Hz}, \mathrm{C}_{\text {quat }}\right), 134.6\left(\mathrm{C}_{\text {quat }}\right), 133.5(\mathrm{CH}=\mathrm{CH}), 129.3$ (2Ar-CH), 128.7 (Ar-CH), $126.9(2 \mathrm{Ar}-\mathrm{CH}), 125.2\left(\mathrm{~d}, J=10.1 \mathrm{~Hz}, \mathrm{C}_{\text {quat }}\right), 121.4(\mathrm{~d}, J=5.3 \mathrm{~Hz}$, $\left.\mathrm{C}_{\text {quat }}\right), 119.2(\mathrm{CH}=\mathrm{CH}), 116.2(\mathrm{~d}, \mathrm{~J}=26.8 \mathrm{~Hz}, \mathrm{Ar}-\mathrm{CH}), 112.9$ (C quat $), 112.7(\mathrm{~d}, \mathrm{~J}=9.6 \mathrm{~Hz}$, Ar- $\mathrm{CH}), 112.4$ (C quat), $106.5(\mathrm{~d}, J=24.5 \mathrm{~Hz}, \mathrm{Ar}-\mathrm{CH}), 52.0\left(\mathrm{OCH}_{3}\right), 49.9\left(\mathrm{CH}_{2}\right) \mathrm{ppm} .{ }^{19} \mathrm{~F}$ $\operatorname{NMR}\left(282 \mathrm{MHz}, \mathrm{CDCl}_{3}\right): \delta=-118.71 \mathrm{ppm}$. HRMS (ESI): calcd. for $\mathrm{C}_{20} \mathrm{H}_{16} \mathrm{FN}_{2} \mathrm{O}_{2}(\mathrm{M}+\mathrm{H})^{+}$ 335.11958; found 335.12021.

\subsubsection{General Procedure for Stille Coupling (Series 11)}

A Schlenk flask was charged with compound 7a (300 mg, $0.84 \mathrm{mmol}, 1$ equiv.), $\mathrm{DMF}(5 \mathrm{~mL})$, vinyl tin derivatives (1.008 mmol, 1.2 equiv.) and $\mathrm{PdCl}_{2}\left(\mathrm{MeCN}_{2}(21.8 \mathrm{mg}\right.$, 
$0.084 \mathrm{mmol}, 10 \mathrm{~mol} \%$ ) under argon. The mixture was stirred at $40{ }^{\circ} \mathrm{C}$ for 1 week. Then, the reaction mixture was added to a mixture of $\mathrm{KF}(2 \mathrm{M})$ and ethyl acetate (50:50), stirred for $30 \mathrm{~min}$ at room temperature and filtered through a Celite ${ }^{\odot}$ pad. Solvents were removed under reduced pressure, and the crude product was purified by column chromatography with a stationary phase composed of $10 \%$ powdered anhydrous $\mathrm{K}_{2} \mathrm{CO}_{3}$ and silica, using petroleum ether/ethyl acetate as eluent (99.9:0.1).

(E)-1-Benzyl-3-(3-methylbut-1-en-1-yl)-1H-indole-2-carbonitrile (11b): Brown oil, 35\%, $88 \mathrm{mg} .{ }^{1} \mathrm{H} \mathrm{NMR}\left(300 \mathrm{MHz}, \mathrm{CDCl}_{3}\right): \delta=7.90(\mathrm{dt}, J=8.2 \mathrm{~Hz}, J=0.9 \mathrm{~Hz}, \mathrm{~Hz}, 1 \mathrm{H}), 7.39-7.26$ $(\mathrm{m}, 5 \mathrm{H}, \mathrm{Ar}-\mathrm{CH}), 7.25-7.13(\mathrm{~m}, 3 \mathrm{H}, \mathrm{Ar}-\mathrm{CH}), 6.63(\mathrm{~d}, J=16.2 \mathrm{~Hz}, 1 \mathrm{H}, \mathrm{CH}=\mathrm{CH}), 6.54(\mathrm{dd}, J=$ $16.2 \mathrm{~Hz}, J=6.2 \mathrm{~Hz}, 1 \mathrm{H}, \mathrm{CH}=\mathrm{CH}), 5.44\left(\mathrm{~s}, 2 \mathrm{H}, \mathrm{CH}_{2}\right), 2.56$ (oct, $\left.J=6.6 \mathrm{~Hz}, 1 \mathrm{H}, \mathrm{CH}\left(\mathrm{CH}_{3}\right)_{2}\right)$, $1.16\left(\mathrm{~d}, J=6.7 \mathrm{~Hz}, 6 \mathrm{H}, 2 x \mathrm{CH}_{3}\right)$ ppm. ${ }^{13} \mathrm{C} \mathrm{NMR}\left(75 \mathrm{MHz} \mathrm{CDCl}_{3}\right): \delta=141.8(\mathrm{CH}=\mathrm{CH}), 138.1$ (C quat $), 136.2$ ( $\left.\mathrm{C}_{\text {quat }}\right), 129.1(2 \mathrm{Ar}-\mathrm{CH}), 128.2(\mathrm{Ar}-\mathrm{CH}), 127.0\left(\mathrm{C}_{\text {quat }}\right), 126.9$ (2Ar-CH), 126.5

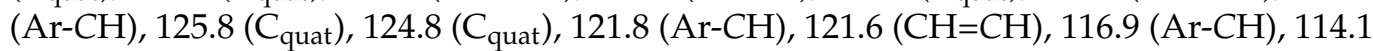
( $\left.\mathrm{C}_{\text {quat }}\right), 110.9(\mathrm{Ar}-\mathrm{CH}), 49.0\left(\mathrm{CH}_{2}\right), 32.5\left(\mathrm{CH}\left(\mathrm{CH}_{3}\right)_{2}\right), 22.6\left(2 \times \mathrm{CH}_{3}\right)$ ppm. HRMS (ESI): calcd. for $\mathrm{C}_{21} \mathrm{H}_{21} \mathrm{~N}_{2}(\mathrm{M}+\mathrm{H})^{+}$301.17047; found 301.17038.

(E)-1-Benzyl-3-(2-(trimethylsilyl)vinyl)-1H-indole-2-carbonitrile (11c): Orange oil, $40 \%, 111 \mathrm{mg} .{ }^{1} \mathrm{H}$ NMR $\left(300 \mathrm{MHz} \mathrm{CDCl}_{3}\right): \delta=8.01(\mathrm{dt}, J=8.2 \mathrm{~Hz}, J=0.8 \mathrm{~Hz}, 1 \mathrm{H}, \mathrm{Ar}-$ $\mathrm{CH}), 7.41-7.23(\mathrm{~m}, 6 \mathrm{H}, \mathrm{Ar}-\mathrm{CH}), 7.17(\mathrm{~d}, J=19.6 \mathrm{~Hz}, 1 \mathrm{H}, \mathrm{CH}=\mathrm{CH}), 7.16(\mathrm{dd}, J=7.9 \mathrm{~Hz}, J=$ $2.2 \mathrm{~Hz}, 2 \mathrm{H}, \mathrm{Ar}-\mathrm{CH}), 6.82(\mathrm{~d}, J=19.5 \mathrm{~Hz}, 1 \mathrm{H}, \mathrm{CH}=\mathrm{CH}), 5.45(\mathrm{~s}, 2 \mathrm{H}), 0.22(\mathrm{~s}, 9 \mathrm{H}, 3 \mathrm{xCH}) \mathrm{ppm}$. ${ }^{13} \mathrm{C} \mathrm{NMR}\left(75 \mathrm{MHz}, \mathrm{CDCl}_{3}\right): \delta=138.0$ (C quat), 135.9 ( $\left.\mathrm{C}_{\text {quat }}\right), 133.6(\mathrm{CH}=\mathrm{CH}), 133.0(\mathrm{Ar}-\mathrm{CH})$, $129.0(2 \mathrm{Ar}-\mathrm{CH}), 128.2(\mathrm{Ar}-\mathrm{CH}), 126.8(2 \mathrm{Ar}-\mathrm{CH}), 126.5(\mathrm{Ar}-\mathrm{CH}), 124.7\left(\mathrm{C}_{\text {quat }}\right), 117.0\left(\mathrm{C}_{\text {quat }}\right)$, 113.8 ( $\left.\mathrm{C}_{\text {quat }}\right), 110.9(\mathrm{Ar}-\mathrm{CH}), 108.5\left(\mathrm{C}_{\text {quat }}\right), 49.0\left(\mathrm{CH}_{2}\right),-1.16\left(3 \times \mathrm{CH}_{3}\right)$ ppm. HRMS (ESI): calcd. for $\mathrm{C}_{21} \mathrm{H}_{23} \mathrm{~N}_{2} \mathrm{Si}(\mathrm{M}+\mathrm{H})^{+}$331.16305; found 335.16298.

\subsubsection{Typical Procedure for Addition of $n$-BuLi on Compound $\mathbf{8 b}$}

A suspension of compound $\mathbf{8 b}(300 \mathrm{mg}, 0.865 \mathrm{mmol})$ in THF $(15 \mathrm{~mL})$ was introduced into a dry, single-necked, round-bottomed flask under argon. The suspension was vigorously stirred, and $n$-BuLi ( $2 \mathrm{mmol}, 2.2$ equiv., $0.17 \mathrm{~mL}, 1.6 \mathrm{M}$ in hexane) was added at $-78{ }^{\circ} \mathrm{C}$. The mixture was stirred magnetically under argon for $4 \mathrm{~h}$ at room temperature. Then, $\mathrm{ZnI}_{2}$ (276mg, $0.865 \mathrm{mmol}, 1$ equiv.) and $\mathrm{I}_{2}$ (241 mg, $0.95 \mathrm{mmol}, 1.1$ equiv.) were added at $-78{ }^{\circ} \mathrm{C}$, and the suspension was stirred overnight under argon at rt. The mixture was basified with $2 \mathrm{~N}$ aq $\mathrm{NaOH}(10 \mathrm{~mL})$, extracted with EtOAc $(10 \mathrm{~mL})$ and washed with $5 \%$ aq $\mathrm{Na}_{2} \mathrm{~S}_{2} \mathrm{O}_{3}(10 \mathrm{~mL})$. The organic layer was dried $\left(\mathrm{MgSO}_{4}\right)$, filtered and concentrated under reduced pressure. The residue was purified by chromatography on silica gel.

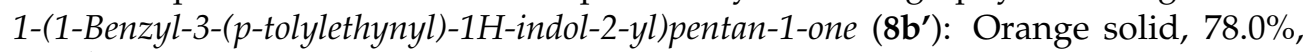
$281 \mathrm{mg} .{ }^{1} \mathrm{H}$ NMR $\left(300 \mathrm{MHz}, \mathrm{CDCl}_{3}\right): \delta=7.95(\mathrm{dt}, J=7.9 \mathrm{~Hz}, J=0.9 \mathrm{~Hz}, 1 \mathrm{H}, \mathrm{Ar}-\mathrm{CH})$, 7.51-7.40 (m, 2H, Ar-CH), 7.30-7.20 (m, 6H, Ar-CH), 7.05-7.02 (m, 2H, Ar-CH), $5.84(\mathrm{~s}, 2 \mathrm{H}$, $\left.\mathrm{CH}_{2}\right), 3.39\left(\mathrm{t}, \mathrm{J}=7.3 \mathrm{~Hz}, 2 \mathrm{H}, \mathrm{CH}_{2}\right), 2.41\left(\mathrm{~s}, 3 \mathrm{H}, \mathrm{CH}_{3}\right), 1.72\left(\mathrm{q}, J=7.5 \mathrm{~Hz}, 2 \mathrm{H}, \mathrm{CH}_{2}\right), 1.37$ (sext, $\left.J=7.6 \mathrm{~Hz}, 2 \mathrm{H}, \mathrm{CH}_{2}\right), 0.91\left(\mathrm{t}, J=7.3 \mathrm{~Hz}, 3 \mathrm{H}, \mathrm{CH}_{3}\right)$ ppm. ${ }^{13} \mathrm{C} \mathrm{NMR}\left(75 \mathrm{MHz}, \mathrm{CDCl}_{3}\right)$ : $\delta=195.2$ ( $\left.\mathrm{C}_{\text {quat }}\right), 138.8$ ( $\left.\mathrm{C}_{\text {quat }}\right), 138.7$ ( $\left.\mathrm{C}_{\text {quat }}\right), 138.2$ ( $\left.\mathrm{C}_{\text {quat }}\right), 135.8$ ( $\left.\mathrm{C}_{\text {quat }}\right), 131.3(2 \mathrm{Ar}-\mathrm{CH})$, $129.4(2 \mathrm{Ar}-\mathrm{CH}), 128.7(2 \mathrm{Ar}-\mathrm{CH}), 128.5\left(\mathrm{C}_{\text {quat }}\right), 127.3(\mathrm{Ar}-\mathrm{CH}), 126.9(\mathrm{Ar}-\mathrm{CH}), 126.5(2 \mathrm{Ar}-\mathrm{CH})$, 122.2 (Ar-CH), 121.8 (Ar-CH), 120.5 (C quat), $111.1(\mathrm{Ar}-\mathrm{CH}), 105.9$ ( $\left.\mathrm{C}_{\text {quat }}\right), 98.5$ ( $\left.\mathrm{C}_{\text {quat }}\right), 85.8$ $\left(\mathrm{C}_{\text {quat }}\right), 48.7\left(\mathrm{CH}_{2}\right), 42.4\left(\mathrm{CH}_{2}\right), 27.0\left(\mathrm{CH}_{2}\right), 22.6\left(\mathrm{CH}_{2}\right), 21.7\left(\mathrm{CH}_{3}\right), 14.1\left(\mathrm{CH}_{3}\right)$ ppm. HRMS (ESI): calcd. for $\mathrm{C}_{29} \mathrm{H}_{28} \mathrm{NO}(\mathrm{M}+\mathrm{H})^{+} 406.21709$; found 406.21720 .

\section{Conclusions}

In summary, we have developed a palladium-catalyzed homocoupling reaction of heteroatoms through $\mathrm{C}-\mathrm{I}$ bond functionalization using different alkynes, alkenes and aryl derivatives. This approach offers a simple strategy and alternative route for the preparation of heteroaryl nitriles from easily available precursors in good to excellent yields. The present work allows access to novel diversely polysubstituted cyanoindoles via Sonogashira, Suzuki-Miyaura, Heck and Stille cross-coupling reactions.

Supplementary Materials: The following are available online, File S1: 1H- and 13C-NMR or J-mod spectra for compounds 3a-d, 4a, 5a-f, 6a-d, 7a-d, 8a-j, 9a-p, 10a-f, 11a-c and 8b'. 
Author Contributions: Conceptualization, A.H.; data curation, Y.C.; formal analysis, A.H., M.R.-Y. and Y.C.; investigation, J.T.; methodology, A.H. and M.C.; project administration, J.T.; validation, Y.C.; resources, J.T.; writing—original draft preparation, J.T., A.H. and M.C.; writing-review and editing, J.T.; visualization, J.T. and Y.C.; supervision, J.T. All authors discussed the results and commented on the manuscript. All authors have read and agreed to the published version of the manuscript.

Funding: This research received no external funding.

Institutional Review Board Statement: Not applicable.

Informed Consent Statement: Not applicable.

Data Availability Statement: The data presented in this sudy ara available in Supplementary Materials.

Acknowledgments: We acknowledge Frédéric Montigny (Analysis Department, Tours University) for HRMS analysis. We acknowledge the French Ministry for Research and Innovation for financial support.

Conflicts of Interest: The authors declare no conflict of interest. The funders had no role in the design of the study; in the collection, analyses or interpretation of data; in the writing of the manuscript or in the decision to publish the results.

Sample Availability: Samples of the compounds 3a-d, 4a, 5a-f, 6a-d, 7a-d, 8a-j, 9a-p, 10a-f, 11a-c and $\mathbf{8} \mathbf{b}^{\prime}$ are available from the authors.

\section{References}

1. Humphrey, G.R.; Kuethe, J.T. Practical methodologies for the synthesis of indoles. Chem. Rev. 2006, 106, 2875-2911. [CrossRef]

2. Katayama, S.; Ae, N.; Nagata, R. Synthesis of tricyclic indole-2-carboxylic acids as potent NMDA-glycine antagonists. J. Org. Chem. 2001, 66, 3474-3483. [CrossRef]

3. Leboho, T.C.; Michael, J.P.; van Otterlo, W.A.L.; van Vuuren, S.F.; de Koning, C.B. The synthesis of 2- and 3-aryl indoles and 1,3,4,5-tetrahydropyran[4,3-b]indoles and their antibacterial and antifungal activity. Bioorg. Med. Chem. Lett. 2009, 19, 4948-4951. [CrossRef]

4. Lounasma, M.; Tolvanen, A. Simple indole alkaloids and those with a nonrearranged monoterpenoid unit. Nat. Prod. Rep. 2000, $17,175-191$.

5. Chen, Z.; Zeng, X.; Yan, B.; Zhao, Y.; Fu, Y. Au-Catalyzed intramolecular annulations toward fused tricyclic [1,3]oxazino[3,4a]indol-1-ones under extremely mild conditions. RSC Adv. 2015, 5, 100251-100255. [CrossRef]

6. de Sa alves, F.R.; Barreiro, E.J.; Fraga, C.A.M. From nature to drug discovery: The indole scaffold as a 'privileged structure'. Mini Rev. Med. Chem. 2009, 9, 782-793. [CrossRef]

7. Chada, N.; Silakari, O. Indoles as therapeutics of interest in medicinal chemistry: Bird's eye view. Eur. J. Med. Chem. 2017, 134, 159-184. [CrossRef]

8. Kumari, A.; Singh, R.K. Medicinal chemistry of indole derivatives: Current to future therapeutic prospectives. Bioorg. Chem. 2019, 89, 103021. [CrossRef]

9. Kovacikova, L.; Prnva, M.S.; Majekova, M.; Bohac, A.; Karasu, C.; Stefek, M. Development of Novel indole-based bifunctional aldose reductase inhibitors/antioxydants as promising drugs for the treatment of diabetic complications. Molecules 2021, $26,2867$. [CrossRef]

10. Festa, A.A.; Zalte, R.R.; Golantsov, N.E.; Varlamov, A.V.; Van der Eycken, E.V.; Voskressensky, L.G. DBU-Catalyzed alkyne-imidate cyclization toward 1-alkoxypyrazino[1,2-a] indolesynthesis. J. Org. Chem. 2018, 83, 9305-9311. [CrossRef]

11. Jaisankar, P.; Srinivasan, P.A. Facile synthesis of 1-phenylsulfonyl-3-substituted-2-cyanoindoles, 1-phenylsulfonyl-2-methyl-3cyanoindoles, and bifunctional 1-phenyl sulfonylindoles. Synthesis 2006, 14, 2413-2417.

12. Golantsov, N.E.; Karchava, A.V.; Nosova, V.M.; Yurovskaya, M.A. Stereoselective synthesis of 4-substituted 1,2,3,4,10,10ahexahydropyrazino[1,2-a]indoles. Russ. Chem. Bull. 2005, 54, 226-230. [CrossRef]

13. Liang, L.-N.; An, R.; Huang, T.; Xu, M.; Hao, X.-J.; Pan, W.-D.; Liu, S. A simple approach for the syntheses of rutaecarpine and its analogs. Tetrahedron Lett. 2015, 56, 2466-2468. [CrossRef]

14. Almagro, L.; Fernández-Pérez, F.; Pedreño, M.A. Indole alkaloids from catharanthus roseus: Bioproduction and their effect on human health. Molecules 2015, 20, 2973-3000. [CrossRef] [PubMed]

15. Hys, V.Y.; Milokhov, D.S.; Keda, T.Y.; Omelchenko, I.V.; Konovalova, I.S.; Shishkina, S.V.; Volovenko, Y.M. Efficient synthesis of seven-membered aza-sultams: Heterofused amino-1,2,4-thiadiazepine dioxides. Tetrahedron 2021, 88, 132149. [CrossRef]

16. Iioka, R.; Yorozu, K.; Sakai, Y.; Kawai, R.; Hatae, N.; Takashima, K.; Tanabe, G.; Wasada, H.; Yoshimatsu, M. Synthesis of azepino[1,2-a]indole-10-amines via [6+1] annulation of ynenitriles with reformatsky reagent. Eur. J. Org. Chem. 2021, 10, 1553-1558. [CrossRef] 
17. Zalte, R.R.; Festa, A.A.; Golantsov, N.E.; Subramani, K.; Rybakov, V.B.; Varlamov, A.V.; Luque, R.; Voskressensky, L.G. Aza-henry and aza-knoevenagel reactions of nitriles for the synthesis of pyrido[1,2-a]indoles. Chem. Commun. 2020, 56, 6527-6530. [CrossRef]

18. Festa, A.; Golantsov, N.; Storozhenko, O.; Shumsky, A.; Varlamov, A.; Voskressensky, L. Alcohol-Initiated dinitrile cyclization in basic media: A route toward pyrazino[1,2-a]indole-3-amines. Synlett 2018, 29, 898-903.

19. Reddy, G.; Kumar, P.; Anand, R.; Mukkanti, K.; Reddy, P. An expedient entry to fused [1,2,5]-triazepinederivatives: Extension to fused [1,2,5]-oxadiazinederivatives, a new class of seven-membered heterocycles. Synlett 2009, 9, 1463-1465. [CrossRef]

20. Moll, A.; Hübner, H.; Gmeiner, P.; Troschütz, R. Phenylpiperazinylmethylindolecarboxylates and derivatives as selective $\mathrm{D}_{4}$-ligands. Bioorg. Med. Chem. 2002, 10, 1671-1679. [CrossRef]

21. Heinz, F.; Lyothier, I.; Pothier, J.; Richard-Bildstein, S.; Thierry, S. N-Substituted Indole Derivatives. Patent WO2018210995, 22 November 2018.

22. Stenzel, W.; Armah, B. Preparation of N-Diarylmethyl-3-[(2-cyanoindol-4-yl)aminopropoxy]azetidines as Cardiovascular agents. Patent DE4002391, 1 August 1991.

23. Lopchuk, J.M.; Montgomery, W.L.; Jasinski, J.P.; Gorjifard, S.; Gribble, G.W. Manganese(III)-mediated oxidative radical addition of malonates to 2-cyanoindoles. Tetrahedron Lett. 2013, 54, 6142-6145. [CrossRef]

24. Huang, L.; Wei, Y.; Shi, M. Asymmetric substitutions of o-boc-protected morita-baylis-hillman adducts with pyrrole and indole derivatives. Org. Biomol. Chem. 2012, 10, 1396-1405. [CrossRef] [PubMed]

25. Borza, I.; Kolok, S.; Ignácz-Szendrei, G.; Greiner, I.; Tárkányi, G.; Galgóczy, K.; Horváth, C.; Farkas, S. Domány, G. Indole-2carboxamidines as novel NR2B selective NMDA receptor antagonists. Bioorg. Med. Chem. Lett. 2005, 15, 5439-5441. [CrossRef]

26. Kaboudin, B.; Esfandiari, H.; Moradi, A.; Kazemi, F.; Aoyama, H. ZnCl 2 -Mediated double addition of dialkylphosphite to nitriles for the synthesis of 1-aminobisphosphonates. J. Org. Chem. 2019, 84, 14943-14948. [CrossRef] [PubMed]

27. Woodroofe, C.C.; Meisenheimer, P.L.; Klaubert, D.H.; Kovic, Y.; Rosenberg, J.C.; Behney, C.E.; Southworth, T.L.; Branchini, B.R. Novel heterocyclic analogues of firefly luciferin. Biochemistry 2012, 51, 9807-9813. [CrossRef] [PubMed]

28. Lu, Y.; Li, C.-M.; Wang, Z.; Chen, J.; Mohler, M.L.; Li, W.; Dalton, J.T.; Miller, D.D. Design, synthesis, and SAR studies of 4-substituted methoxylbenzoyl-aryl-thiazoles analogues as potent and orally bioavailable anticancer agents. J. Med. Chem. 2011, 54, 4678-4693. [CrossRef] [PubMed]

29. Larock, R.C. Comprehensive Organic Transformations: A Guide to Functional Group Preparations; Wiley: New York, NY, USA, 1999.

30. Zhao, M.; Zhang, W.; Shen, Z. Cu-Catalyzed cyanation of indoles with acetonitrile as a cyano source. J. Org. Chem. 2015, 80, 8868-8873. [CrossRef] [PubMed]

31. Xu, S.; Huang, X.; Hong, X.; Xu, B. Palladium-assisted regioselective C-H cyanation of heteroarenes using isonitrile as cyanide source. Org. Lett. 2012, 14, 4614-4617. [CrossRef]

32. Nagase, Y.; Sugiyama, T.; Nomiyama, S.; Yonekura, K.; Tsuchimoto, T. Zinc-Catalyzed direct cyanation of indoles and pyrroles: Nitromethane as a source of a cyano group. Adv. Synth. Catal. 2014, 356, 347-352. [CrossRef]

33. Yuen, O.Y.; Choy, P.Y.; Chow, W.K.; Wong, W.T.; Kwong, F.Y. Synthesis of 3-cyanoindole derivatives mediated by copper(I) iodide using benzyl cyanide. J. Org. Chem. 2013, 78, 3374-3378. [CrossRef]

34. Liu, W.; Richter, S.C.; Mei, R.; Feldt, M.; Ackermann, L. Synergistic heterobimetallic manifold for expedient manganese(I)catalyzed C-H cyanation. Chem. Eur. J. 2016, 22, 17958-17961. [CrossRef]

35. Zeidan, N.; Bognar, S.; Lee, S.; Lautens, M. Palladium-Catalyzed synthesis of 2-cyanoindoles from 2-gem-dihalovinylanilines. Org. Lett. 2017, 19, 5058-5061. [CrossRef]

36. Cohen, D.T.; Buchwald, S.L. Mild palladium-catalyzed cyanation of (hetero)aryl halides and triflates in aqueous media. Org. Lett. 2015, 17, 202-205. [CrossRef]

37. Ruiz-Castillo, P.; Buchwald, S.L. Applications of palladium-catalyzed C-N cross-coupling reactions. Chem. Rev. 2016, 116, 12564-12649. [CrossRef]

38. Sain, S.; Jain, S.; Srivastava, M.; Vishwakarma, R.; Dwivedi, J. Application of palladium-catalyzed cross-coupling reactions in organic synthesis. Curr. Org. Chem. 2019, 16, 1105-1142. [CrossRef]

39. McGlinchey, M.J.; Nikitin, K. Palladium-catalysed coupling reactions en route to molecular machines: Sterically hindered indenyl and ferrocenyl anthracenes and triptycenes, and biindenyls. Molecules 2020, 25, 1950. [CrossRef]

40. Cacchi, S.; Fabrizi, G. Update 1: Synthesis and functionalization of indoles through palladium-catalyzed reactions. Chem. Rev. 2011, 111, PR215-PR283. [CrossRef]

41. Torborg, C.; Beller, M. Recent applications of palladium-catalyzed coupling reactions in the pharmaceutical, agrochemical, and fine chemical industries. Adv. Synth. Catal. 2009, 351, 3027-3043. [CrossRef]

42. Khairul, W.M.; Daud, A.I.; Hanifaah, N.A.M.; Arshad, S.; Razak, I.A.; Zuki, H.M.; Erben, M.F. Structural study of a novel acetylide-thiourea derivative and its evaluation as a detector of benzene. J. Mol. Struct. 2017, 1139, 353-361. [CrossRef]

43. Buchmeiser, M.R.; Schareina, T.; Kempe, R.; Wurst, K. Bis(pyrimidine)-based palladium catalysts: Synthesis, X-ray structure and applications in Heck-, Suzuki-, Sonogashira-Hagihara couplings and amination reactions. J. Organomet. Chem. 2001, 634, 39-46. [CrossRef]

44. Durust, Y.; Sagirli, A.; Kariuki, B.M.; Knight, D.W. [1,3]-Dipolar cycloaddition of N-aryl sydnones to benzothiophene 1,1-dioxide, 1-cyclopropylprop-2-yn-1-ol and 1-(prop-2-ynyl)-1H-indole. Tetrahedron 2014, 70, 6012-6019. [CrossRef]

45. Sakamoto, T.; Nagano, T.; Kondo, Y.; Yamanaka, H. Palladium-catalyzed coupling reaction of 3-iodoindoles and 3iodobenzo[b]thiophene with terminal acetylenes. Chem. Pharm. Bull. 1988, 36, 2248-2252. [CrossRef] 
46. Inack-Ngi, S.; Guilloteau, V.; Abarbri, M.; Thibonnet, J. Regioselective copper-mediated synthesis of thieno[2,3-c]pyrane-7-one, indolo[2,3-c]pyrane-1-one, and indolo[3,2-c]pyrane-1-one. J. Org. Chem. 2011, 76, 8347-8354. [CrossRef] [PubMed]

47. Evano, G.; Blanchard, N.; Toumi, M. Copper-mediated coupling reactions and their applications in natural products and designed biomolecules synthesis. Chem. Rev. 2008, 108, 3054-3131. [CrossRef]

48. Liang, B.; Dai, M.; Chen, J.; Yang, Z. Copper-free sonogashira coupling reaction with $\mathrm{PdCl}_{2}$ in water under aerobic conditions. J. Org. Chem. 2005, 70, 391-393. [CrossRef]

49. Enguehard-Gueiffier, C.; Delaye, P.-O.; Penichon, M.; Denevault-Sabourin, C.; Allouchi, H.; Gueiffier, A. Domino 6-endo-dig cyclization/halogenation reactions: Three-component synthesis of 1,3-disubstituted 4-haloimidazo[1,2-a:4,5-c' $c^{\prime}$ dipyridines. Synthesis 2015, 24, 3983-3989. [CrossRef]

50. Jana, R.; Pathak, T.P.; Sigman, M.S. Advances in transition metal (Pd,Ni,Fe)-catalyzed cross-coupling reactions using alkylorganometallics as reaction partners. Chem. Rev. 2011, 111, 1417-1492. [CrossRef] [PubMed]

51. Beaumont, S.; Retailleau, P.; Dauban, P.; Dodd, R.H. Synthesis of indolobenzazepinones by application of an isocyanide-based multicomponent reaction. Eur. J. Org. Chem. 2008, 2008, 5162-5175. [CrossRef]

52. Santra, S.; Hota, P.K.; Bhattacharyya, R.; Bera, P.; Ghosh, P.; Mandal, S.K. Palladium nanoparticles on graphite oxide: A recyclable catalyst for the synthesis of biaryl cores. ACS Catal. 2013, 3, 2776-2789. [CrossRef]

53. Beletskaya, I.P.; Cheprakov, A.V. The Heck reaction as a sharpening stone of palladium catalysis. Chem. Rev. 2000, 100, 3009-3066. [CrossRef]

54. Stille, J.K. The palladium-catalyzed cross-coupling reactions of organotin reagents with organic electrophiles. Angew. Chem. Int. Ed. 1986, 25, 508-524. [CrossRef]

55. Hammoud, S.; Anselmi, E.; Cherry, K.; Kizirian, J.-C.; Thibonnet, J. Synthesis and reactivity of oxazinoindolones via regioselective 6-exo-dig iodolactonization. Eur. J. Org. Chem. 2018, 2018, 6314-6327. [CrossRef]

56. Harrowven, D.C.; Curran, D.P.; Kostiuk, S.L.; Wallis-Guy, I.L.; Whiting, S.; Stenning, K.J.; Tang, B.; Packard, E.; Nanson, L. Potassium carbonate-silica: A highly effective stationary phase for the chromatographic removal of organotin impurities. Chem. Commun. 2010, 46, 6335-6337. [CrossRef] [PubMed]

57. Armarego, W.L.F. Chai. In Purification of Laboratory Chemicals, 7th ed.; Butterworth-Heinemann: Oxford, UK, 2013.

58. Seyferth, D.; Stone, F.G.A. Vinyl derivatives of the metals. I. Synthesis of vinyltin compounds. J. Am. Chem. Soc. 1957, 79, 515-517. [CrossRef]

59. Saihi, M.L.; Pereyre, M. Reactivity of vinylic organotin compounds towards acid chlorides and anhydrides and $\alpha$-halo esters. Bulletin de la Société Chimique de France 1977, 9, 1251-1255. [CrossRef]

60. Cunico, R.F.; Clayton, F.J. Trans-.beta.-trimethylsilylvinyllithium. J. Org. Chem. 1976, 41, 1480-1482. [CrossRef]

61. Chong, J.M.; Park, S.B. Stereoselective synthesis of (E)-vinylstannanes from aldehydes. J. Org. Chem. 1993, 58, 523-527. [CrossRef] 